\title{
BOUNDARY SEPARATED AND CLUSTERED LAYER POSITIVE SOLUTIONS FOR AN ELLIPTIC NEUMANN PROBLEM WITH LARGE EXPONENT
}

\author{
YIBIN ZHANG
}

\begin{abstract}
Given a smooth bounded domain $\mathcal{D}$ in $\mathbb{R}^{N}$ with $N \geq 3$, we study the existence and the profile of positive solutions for the following elliptic Nenumann problem

$$
\begin{cases}-\Delta v+v=v^{p}, \quad v>0 & \text { in } \mathcal{D}, \\ \frac{\partial v}{\partial \nu}=0 & \text { on } \partial \mathcal{D},\end{cases}
$$

where $p>1$ is a large exponent and $\nu$ denotes the outer unit normal vector to the boundary $\partial \mathcal{D}$. For suitable domains $\mathcal{D}$, by a constructive way we prove that, for any non-negative integers $k, l$ with $k+l \geq 1$, if $p$ is large enough, such a problem has a family of positive solutions with $k$ boundary layers and $l$ interior layers which concentrate along $k+l$ distinct $(N-2)$-dimensional minimal submanifolds of $\partial \mathcal{D}$, or collapse to the same $(N-2)$-dimensional minimal submanifold of $\partial \mathcal{D}$ as $p \rightarrow+\infty$.
\end{abstract}

\section{INTRODUCTION}

In this paper, we are interested in the following classical elliptic Neumann problem

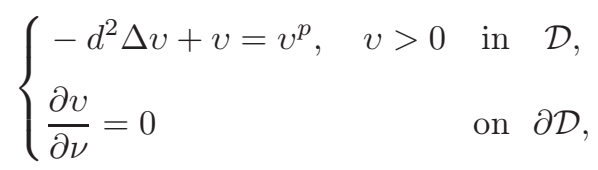

where $\mathcal{D}$ is a smooth bounded domain in $\mathbb{R}^{N}, N \geq 2, d>0$ is a parameter, $p>1$ is a exponent and $\nu$ denotes the outer unit normal vector to the boundary $\partial \mathcal{D}$.

Problem (1.1) has received considerable attention in the last three decades, because it appears in many different mathematical models: for example, it arises from the study of steady states for the logarithmic Keller-Segel system in chemotaxis [33] and the shadow system of the Gierer-Meinhardt model in biological pattern formation [23]. In particular, it has been shown that the solutions of (1.1) exhibit a variety of interesting concentration phenomena as either the exponent $p$ tends to some critical values or the parameter $d$ tends to zero.

Let us first define $p_{h+1}^{*}:=(N-h+2) /(N-h-2), 0 \leq h \leq N-3$ as the $(h+1)$-th critical exponent (recall that $h$ is an integer) and set $p_{N-1}^{*}:=+\infty$. In the subcritical case, i.e. $N=2$, or $N \geq 3$ and $p<p_{1}^{*}=(N+2) /(N-2)$, compactness of Sobolev's embedding ensures the existence of a positive least energy solution of (1.1). For $d>0$ sufficiently small, Lin, Ni and Takagi in [34, 46, 47] proved that this least energy solution has exactly one bounded, very sharp spike located on the boundary and near the most curved part of the boundary, i.e., the region where the mean curvature of the boundary attains its maximum. Higher energy solutions of (1.1) with multiple boundary peaks as well as multiple interior peaks have been established in $[12,13,14,20,25,29,30,31,32,36,56,58]$. It turns out that multiple boundary spikes tend to cluster around critical points of the mean curvature of the boundary, while the location of multiple interior spikes is determined by the distance between the peaks and the boundary. In particular, Gui and Wei in [30] proved that for any non-negative integers $k, l$ with $k+l \geq 1$, problem (1.1) has a solution with exactly $k$ different boundary spikes and $l$ different interior spikes provided that $d$ is small and $p$ is subcritical. Generally, such spiky solutions are called solutions with 0-dimensional concentration sets.

2010 Mathematics Subject Classification. Primary 35B25; Secondary 35B38, 35J25.

Key words and phrases. Boundary separated and clustered layer positive solutions; Large exponent; Anisotropic Green's function. 
YIBIN ZHANG

In the critical case, i.e. $p=p_{1}^{*}$, such type of concentration phenomena occurs, but the situation is quite different. The lack of compactness of Sobolev's embedding makes it non-obvious to apply variational techniques to obtain a nonconstant least energy solution of (1.1) when $d$ is sufficiently small. The first existence result for a nonconstant least energy solution of (1.1) in general domains and $d$ small were obtained by Adimurthi-Mancini [1] and Wang [55]. The profile and asymptotic behavior of this least energy solution has been clarified in the subsequent works $[4,45,49]$. As in the subcritical case, the least energy solution has a unique maximum point or peak that lies on the boundary and goes, as $d$ tends to zero, to a maximum point of the mean curvature of the boundary. Unlike the subcritical case, the least energy solution blows up, as $d$ tends to zero, at a maximum point of the mean curvature of the boundary. Higher energy solutions with one or more separated or clustered boundary blow-up points have been exhibited for instance in $[2,3,24,27,35,49,50,59]$, where these blow-up points are nothing but degenerate or non-degenerate critical points of the mean curvature of the boundary. A major difference with the subcritical case is that the condition of positivity for the mean curvature at these critical points turns out to be necessary for the boundary bubbling phenomenon to take place [28, 49]. Note that in striking contrast with the subcritical case, there are no solutions blowing up at only interior points when $d$ tends to zero, namely at least one blow-up point has to lie on the boundary, even all of the blow-up points for solutions with uniformly bounded energy have to lie only on the boundary as established in [10, 21, 51]. Moreover, for the subcritical case, spiky solutions with only interior peaks always exist, but these interior peaks must stay in the domain as $d$ tends to zero [57]; while for the critical case, interior peaks of solutions could not remain in the domain as $d$ tends to zero [24, 26].

The almost first critical case, i.e. $p=p_{1}^{*} \pm \epsilon$ with $\epsilon$ positive and sufficiently small, has been widely studied. In the slightly subcritical case, i.e. $p=p_{1}^{*}-\epsilon$, the existence of solutions with simple or non-simple blow-up points located on the boundary near critical points of the mean curvature of the boundary with negative value as $\epsilon \rightarrow 0$ for fixed $d$ and $N \geq 4$ was established in [11, 52, 54]; while in the slightly supercritical case, i.e. $p=p_{1}^{*}+\epsilon$, it was proved in $[17,52,54]$ that there also exists a solution with simple or non-simple blow-up points located on the boundary near critical points of the mean curvature of the boundary with positive value as $\epsilon \rightarrow 0$ for fixed $d$ and $N \geq 4$. In [53], it was proved that if $N=3$ and $\epsilon$ tends to zero from below or above, then a solution with one interior blow-up point may exist for finite $d$. Moreover, if $N=2, d$ is fixed and the exponent $p$ goes to $+\infty$, Musso and Wei in [43] proved that for any non-negative integers $k, l$ with $k+l \geq 1$, problem (1.1) admits a solution with exactly $k$ different boundary spikes and $l$ different interior spikes, whose location can be characterized by critical points of a certain combination of Green's function and its regular part.

It seems natural to ask if problem (1.1) has solutions that exhibit concentration phenomena on h-dimensional subsets of $\overline{\mathcal{D}}$ for every $1 \leq h \leq N-1$, as conjectured by Ni in [44]. In particular, given an $h$-dimensional submanifold $\Gamma$ of $\partial \mathcal{D}$ and assuming that either $h \geq N-2$ or $p \leq p_{h+1}^{*}+\epsilon$ with $\epsilon$ positive and sufficiently small, one question is to ask whether problem (1.1) admits a solution that concentrates along $\Gamma$ as either $d$ or $\epsilon$ tends to zero. For results in this direction, we first mention the $(h+1)$-th subcritical case, i.e. $p<p_{h+1}^{*}$, where Malchiodi and Montenegro [40, 41] proved that, given any $N \in \mathbb{N}$ and any $p>1$, there exist solutions concentrating on the whole boundary if the sequence $d$ satisfies some gap condition, corresponding to $h=N-1$. Furthermore, the result was extended in $[38,39]$ for general $h$, and the concentration set $\Gamma$ is an embedded closed minimal submanifold of $\partial \mathcal{D}$ which is in addition nondegenerate in the sense that its Jacobi operator is invertible. Indeed, this phenomenon is rather subtle compared with pointwise concentration: existence can only be achieved along a sequence of parameters $d=d_{i} \rightarrow 0$. The sequence of parameters $d_{i}$ must be suitable away from certain values where resonance occurs, and the topological type of the solution changes: unlike the pointwise concentration, the Morse index of these solutions is very large and grows as $d_{i} \rightarrow 0$. Del Pino, Mahmoudi and Musso [16] extended this type of results to the $(h+1)$-th critical case $p=p_{h+1}^{*}$ and proved that if $\Gamma$ is an embedded closed minimal submanifold of $\partial \mathcal{D}$ with dimension $h \leq N-7$ (in particular, $N \geq 8$ ) which is nondegenerate, and a certain weighted average of sectional curvature of $\partial \mathcal{D}$ is positive along $\Gamma$, then problem (1.1) admits a solution for a suitable sequence of parameters $d_{i} \rightarrow 0$ which blows up along $\Gamma$. Further for the almost $(h+1)$-th critical case $p=p_{h+1}^{*} \pm \epsilon$ with $0<\epsilon \rightarrow 0$ but $d$ fixed, the same conclusion was established by Deng, Mahmoudi and Musso [19] under analogous assumptions. Meanwhile, for any integer $h=1, \ldots, N-3$, Manna and Pistoia [42] proved that in some suitable domains $\mathcal{D}$, problem (1.1) has a solution 
which blows up along an $h$-dimensional minimal submanifold $\Gamma$ of $\partial \mathcal{D}$ as $p$ approaches from either below or above the $(h+1)$ th critical exponent $p_{h+1}^{*}$.

In the present paper we consider the almost $(N-1)$-th critical case, i.e. $h=N-2$, and give a positive answer when $d$ is fixed and $p$ goes to $+\infty$. More precisely, we find some domains $\mathcal{D}$ such that if $d=1$ and $p$ is large enough, then for any positive integer $m$, problem (1.1) has a positive solution with $m$ distinct mixed interior and boundary layers which concentrate along $m$ distinct $(N-2)$-dimensional minimal submanifolds of $\partial \mathcal{D}$, or collapse to the same $(N-2)$-dimensional minimal submanifold of $\partial \mathcal{D}$ as $p$ goes to $+\infty$.

Let $\Omega$ be a smooth bounded domain in $\mathbb{R}^{2}$ such that

$$
\bar{\Omega} \subset\left\{\left(x_{1}, x_{2}\right) \in \mathbb{R}^{2} \mid x_{1}>0\right\} \quad \text { or } \quad \bar{\Omega} \subset\left\{\left(x_{1}, x_{2}\right) \in \mathbb{R}^{2} \mid x_{1}, x_{2}>0\right\} .
$$

Let $n=1$ or $n=2$ be fixed. Fix $k_{1}, k_{n} \in \mathbb{N}$ with $h:=k_{1}+k_{n}=N-2$ and set

$$
\mathcal{D}:=\left\{\left(y_{1}, y_{n}, x^{\prime}\right) \in \mathbb{R}^{k_{1}+1} \times \mathbb{R}^{k_{n}+1} \times \mathbb{R}^{2-n} \mid\left(\left|y_{1}\right|,\left|y_{n}\right|, x^{\prime}\right) \in \Omega\right\} .
$$

Then $\mathcal{D}$ is a smooth bounded domain in $\mathbb{R}^{N}$ which is $\Upsilon$-invariant for the action of the group $\Upsilon:=\mathcal{O}\left(k_{1}+1\right) \times \mathcal{O}\left(k_{n}+1\right)$ on $R^{N}$ given by

$$
\left(g_{1}, g_{n}\right)\left(y_{1}, y_{n}, x^{\prime}\right):=\left(g_{1} y_{1}, g_{n} y_{n}, x^{\prime}\right) .
$$

Here $\mathcal{O}\left(k_{i}+1\right)$ denotes the group of linear isometries of $\mathbb{R}^{k_{i}+1}$. For $p>1$ large enough we shall look for $\Upsilon$-invariant solutions of problem (1.1) with $d=1$, i.e. solutions $v$ of the form

$$
v\left(y_{1}, y_{n}, x^{\prime}\right)=u\left(\left|y_{1}\right|,\left|y_{n}\right|, x^{\prime}\right) .
$$

Then a simple calculation shows that $v$ solves problem (1.1) with $d=1$ if and only if $u$ satisfies

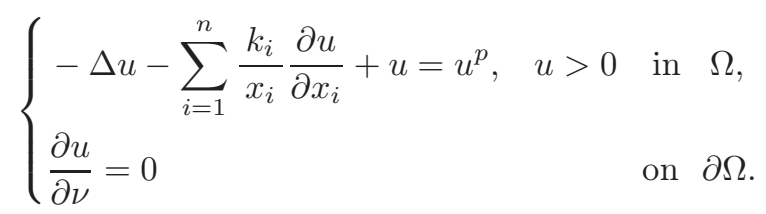

Thus, we are led to study the more general anisotropic problem

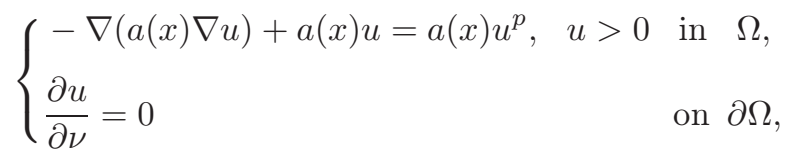

where $\Omega$ is a smooth bounded domain in $\mathbb{R}^{2}, a(x)$ is a positive smooth function over $\bar{\Omega}, p>1$ is a large exponent and $\nu$ denotes the outer unit normal vector to $\partial \Omega$. Note that if

$$
a(x)=a\left(x_{1}, x_{n}, x^{\prime}\right):=x_{1}^{k_{1}} x_{n}^{k_{n}},
$$

then problem (1.5) can be rewritten as equation (1.4).

Our goal is to construct solutions $u_{p}$ to problem (1.5) with $m$ distinct mixed interior and boundary spikes which concentrate at points $\xi_{1}^{*}, \ldots, \xi_{m}^{*}$ of $\partial \Omega$, or accumulate to the same point $\xi_{*}$ of $\partial \Omega$ as $p$ goes to $+\infty$. They correspond, via (1.3), to $\Upsilon$-invariant solutions $v_{p}$ of problem (1.1) $\left.\right|_{d=1}$ with $m$ distinct mixed interior and boundary layers which concentrate along the $\Upsilon$-orbits $\Xi\left(\xi_{1}^{*}\right), \ldots, \Xi\left(\xi_{m}^{*}\right)$ of $\partial \mathcal{D}$, or collapse to the same $\Upsilon$-orbit $\Xi\left(\xi_{*}\right)$ of $\partial \mathcal{D}$ as $p$ goes to $+\infty$. Here

$$
\Xi(\zeta):=\left\{\left(y_{1}, y_{n}, x^{\prime}\right) \in \partial \mathcal{D} \mid\left(\left|y_{1}\right|,\left|y_{n}\right|, x^{\prime}\right)=\zeta \in \partial \Omega\right\}
$$

is a $(N-2)$-dimensional minimal submanifold of $\partial \mathcal{D}$ diffeomorphic to $\mathbb{S}^{k_{1}} \times \mathbb{S}^{k_{n}}$ (note that $k_{1}+k_{n}=h=N-2$ ), where $\mathbb{S}^{k_{i}}$ is the unit sphere in $\mathbb{R}^{k_{i}+1}$.

Let us define the linear differential operator

$$
\Delta_{a} u=\frac{1}{a(x)} \nabla(a(x) \nabla u)=\Delta u+\nabla \log a(x) \nabla u
$$


and the Green's function associated with the Neumann problem

$$
\begin{cases}-\Delta_{a} G(x, y)+G(x, y)=\delta_{y}(x), & x \in \Omega, \\ \frac{\partial G}{\partial \nu_{x}}(x, y)=0, & x \in \partial \Omega,\end{cases}
$$

for every $y \in \bar{\Omega}$. The regular part of $G(x, y)$ is defined depending on whether $y$ lies in the domain or on its boundary as

$$
H(x, y)= \begin{cases}G(x, y)+\frac{1}{2 \pi} \log |x-y|, & y \in \Omega, \\ G(x, y)+\frac{1}{\pi} \log |x-y|, & y \in \partial \Omega .\end{cases}
$$

Our first result concerns the existence of solutions of problem (1.5) whose interior and boundary spikes are uniformly far away from each other and interior spikes lie in the domain with distance to the boundary uniformly approaching zero.

Theorem 1.1. Let $k, l$ be non-negative integers with $m=k+l \geq 1$ and assume that there exist $m$ different points $\xi_{1}^{*}, \ldots, \xi_{m}^{*} \in \partial \Omega$ such that each $\xi_{i}^{*}$ is either a strict local maximum or a strict local minimum point of a $(x)$ on $\partial \Omega$ and satisfies for all $i=1, \ldots, l, \partial_{\nu} a\left(\xi_{i}^{*}\right):=\left\langle\nabla a\left(\xi_{i}^{*}\right), \nu\left(\xi_{i}^{*}\right)\right\rangle>0$. Then, there exists $p_{0}$ such that for any $p>p_{0}$, there is a family of positive solutions $u_{p}$ for problem (1.5) with $k$ different boundary spikes and $l$ different interior spikes located at distance $O(1 / p)$ from $\partial \Omega$. More precisely,

$$
u_{p}(x)=\sum_{i=1}^{m} \frac{1}{\gamma \mu_{i}^{2 /(p-1)}}\left[\log \frac{1}{\left(\delta_{i}^{2}+\left|x-\xi_{i}^{p}\right|^{2}\right)^{2}}+c_{i} H\left(x, \xi_{i}^{p}\right)\right]+o(1),
$$

where $o(1) \rightarrow 0$, as $p \rightarrow+\infty$, on each compact subset of $\bar{\Omega} \backslash\left\{\xi_{1}^{p}, \ldots, \xi_{m}^{p}\right\}$, the parameters $\gamma, \delta_{i}$ and $\mu_{i}$ satisfy

$$
\gamma=p^{p /(p-1)} \varepsilon^{2 /(p-1)}, \quad \quad \delta_{i}=\mu_{i} \varepsilon, \quad \varepsilon=e^{-p / 4}, \quad \frac{1}{C}<\mu_{i}<C p^{2\left(m^{2}+1\right)},
$$

for some $C>0,\left(\xi_{1}^{p}, \ldots, \xi_{m}^{p}\right) \in \Omega^{l} \times(\partial \Omega)^{k}$ satisfies

$$
\xi_{i}^{p} \rightarrow \xi_{i}^{*} \quad \text { for all } i, \quad \text { and } \quad \operatorname{dist}\left(\xi_{i}^{p}, \partial \Omega\right)=O(1 / p) \quad \forall i=1, \ldots, l,
$$

and $c_{i}=8 \pi$ for $i=1, \ldots, l$, but $c_{i}=4 \pi$ for $i=l+1, \ldots, m$. In particular, for any $d>0$, as $p \rightarrow+\infty$,

$$
\begin{gathered}
p u_{p}^{p+1}-8 \pi e \sum_{i=1}^{l} \delta_{\xi_{i}^{*}}+4 \pi e \sum_{i=l+1}^{m} \delta_{\xi_{i}^{*}} \quad \text { weakly in the sense of measures in } \bar{\Omega}, \\
u_{p} \rightarrow 0 \quad \text { uniformly in } \bar{\Omega} \backslash \bigcup_{i=1}^{m} B_{d}\left(\xi_{i}^{*}\right),
\end{gathered}
$$

and

$$
\sup _{x \in \bar{\Omega} \cap B_{d}\left(\xi_{i}^{*}\right)} u_{p}(x) \rightarrow \sqrt{e} .
$$

The corresponding result for problem (1.1) $\left.\right|_{d=1}$ can be stated as follows.

Theorem 1.2. Let $k, l$ be non-negative integers with $k+l \geq 1$ and $\mathcal{D}$ be as in (1.2). If the assumption of Theorem 1.1 holds, then there exists $p_{0}$ such that for any $p>p_{0}$, problem $\left.(1.1)\right|_{d=1}$ has a positive solution $v_{p}$ with $k$ boundary layers and $l$ interior layers which concentrate along $k+l$ different $(N-2)$-dimensional minimal submanifolds of $\partial \mathcal{D}$, namely the $\Upsilon$-orbit $\Xi\left(\xi_{i}^{*}\right)$ of $\xi_{i}^{*}$ for every $i=1, \ldots, k+l$, as $p \rightarrow+\infty$.

Our next result concerns the existence of solutions of problem (1.5) with mixed interior and boundary spikes which accumulate to the same point of the boundary. 
Theorem 1.3. Let $k, l$ be non-negative integers with $m=k+l \geq 1$ and assume that $\xi_{*} \in \partial \Omega$ is a strict local maximum point of $a(x)$ and satisfies $\partial_{\nu} a\left(\xi_{*}\right):=\left\langle\nabla a\left(\xi_{*}\right), \nu\left(\xi_{*}\right)\right\rangle=0$. Then, there exists $p_{0}$ such that for any $p>p_{0}$, there is a family of positive solutions $u_{p}$ for problem (1.5) with $k$ different boundary spikes and $l$ different interior spikes which accumulate to $\xi_{*}$ as $p \rightarrow+\infty$. More precisely,

$$
u_{p}(x)=\sum_{i=1}^{m} \frac{1}{\gamma \mu_{i}^{2 /(p-1)}}\left[\log \frac{1}{\left(\delta_{i}^{2}+\left|x-\xi_{i}^{p}\right|^{2}\right)^{2}}+c_{i} H\left(x, \xi_{i}^{p}\right)\right]+o(1),
$$

where $o(1) \rightarrow 0$, as $p \rightarrow+\infty$, on each compact subset of $\bar{\Omega} \backslash\left\{\xi_{1}^{p}, \ldots, \xi_{m}^{p}\right\}$, the parameters $\gamma, \delta_{i}$ and $\mu_{i}$ satisfy

$$
\gamma=p^{p /(p-1)} \varepsilon^{2 /(p-1)}, \quad \quad \delta_{i}=\mu_{i} \varepsilon, \quad \varepsilon=e^{-p / 4}, \quad \frac{1}{C}<\mu_{i}<C p^{2\left(m^{2}+1\right)},
$$

for some $C>0,\left(\xi_{1}^{p}, \ldots, \xi_{m}^{p}\right) \in \Omega^{l} \times(\partial \Omega)^{k}$ satisfies

$$
\xi_{i}^{p} \rightarrow \xi_{*} \quad \text { for all } i, \quad\left|\xi_{i}^{p}-\xi_{k}^{p}\right|>p^{-2\left(m^{2}+1\right)} \quad \forall i \neq k, \quad \text { and } \quad \operatorname{dist}\left(\xi_{i}^{p}, \partial \Omega\right)>p^{-2\left(m^{2}+1\right)} \quad \forall i=1, \ldots, l,
$$
and $c_{i}=8 \pi$ for $i=1, \ldots, l$, but $c_{i}=4 \pi$ for $i=l+1, \ldots, m$. In particular, for any $d>0$, as $p \rightarrow+\infty$,

and

$$
\begin{gathered}
p u_{p}^{p+1} \rightarrow 4 \pi e(k+2 l) \delta_{\xi_{*}} \quad \text { weakly in the sense of measures in } \bar{\Omega}, \\
u_{p} \rightarrow 0 \quad \text { uniformly in } \bar{\Omega} \backslash B_{d}\left(\xi_{*}\right),
\end{gathered}
$$

$$
\sup _{x \in \bar{\Omega} \cap B_{d / p^{4\left(m^{2}+1\right)}}\left(\xi_{i}^{p}\right)} u_{p}(x) \rightarrow \sqrt{e} .
$$

The corresponding result for problem (1.1) $\left.\right|_{d=1}$ can be stated as follows.

Theorem 1.4. Let $k, l$ be non-negative integers with $k+l \geq 1$ and $\mathcal{D}$ be as in (1.2). If the assumption of Theorem 1.3 holds, then there exists $p_{0}$ such that for any $p>p_{0}$, problem $\left.(1.1)\right|_{d=1}$ has a positive solution $v_{p}$ with $k$ boundary layers and $l$ interior layers which collapse to the same $(N-2)$-dimensional minimal submanifold of $\partial \mathcal{D}$, namely the $\Upsilon$-orbit $\Xi\left(\xi_{*}\right)$ of $\xi_{*}$, as $p \rightarrow+\infty$.

Let us remark that the assumptions in Theorems 1.3-1.4 contain the following two cases:

(A1) $\xi_{*} \in \partial \Omega$ is a strict local maximum point of $a(x)$ restricted on $\partial \Omega$;

(A2) $\xi_{*} \in \partial \Omega$ is a strict local maximum point of $a(x)$ restricted in $\Omega$ and satisfies $\partial_{\nu} a\left(\xi_{*}\right)=\left\langle\nabla a\left(\xi_{*}\right), \nu\left(\xi_{*}\right)\right\rangle=0$.

In fact, arguing as for the proof of Theorem 1.3, we can easily find that if (A1) holds, then problem (1.5) has positive solutions with arbitrarily many boundary spikes which accumulate to $\xi_{*}$ along $\partial \Omega$; while if (A2) holds, then problem (1.5) has positive solutions with arbitrarily many interior spikes which accumulate to $\xi_{*}$ along the inner normal direction of $\partial \Omega$. For the latter case, it seems that this paper is the first one in the literature obtaining this type of concentration phenomenon for positive solutions of some two-dimensional anisotropic nonlinear elliptic Neumann problems, see [5] as an instance.

The general strategy for proving our main results relies on a very well known Lyapunov-Schmidt reduction. In Section 2 we provide an appropriate approximation for a solution of problem (1.5) and give a basic estimate for the scaling error term created by the choice of our approximation. Then we rewrite problem (1.1) in terms of a linearized operator for which a solvability theory, subject to suitable orthogonality conditions, is performed through solving a linearized problem in Section 3. In Section 4 we solve an auxiliary nonlinear problem. In Section 5 we reduce the problem of finding spike solutions of (1.5) to that of finding a critical point of a finite-dimensional function. Section 6 concerns with an asymptotic expansion for the finite-dimensional function appeared in Section 5. Finally, in Section 7 we provide the detailed proof of Theorems 1.1 and 1.3 .

In this paper, the letter $C$ will always denote a generic positive constant independent of $p$, which could be changed from one line to another. The symbol $o(t)$ (respectively $O(t)$ ) will denote a quantity for which $\frac{o(t)}{|t|}$ tends to zero (respectively, $\frac{O(t)}{|t|}$ stays bounded ) as parameter $t$ goes to zero. Moreover, we will use the notation $o(1)$ (respectively $O(1)$ ) to stand for a quantity which tends to zero (respectively, which remains uniformly bounded) as $p \rightarrow+\infty$. 


\section{An APPROXimation FOR THE SOLUtion}

In this section we provide an appropriate approximation for a solution of problem (1.5) and give a basic estimate for the scaling error term created by the choice of our approximation. Since the function $H(x, y)$ defined in (1.7) plays an essential role in our construction, we shall first state its asymptotic behavior without proof, see [5] for details.

Consider the vector function $T(x)=\left(T_{1}(x), T_{2}(x)\right)$ as the solution of

$$
\Delta_{x} T-T=\frac{x}{|x|^{2}} \quad \text { in } \mathbb{R}^{2}, \quad T(x) \in L_{l o c}^{\infty}\left(\mathbb{R}^{2}\right) .
$$

Then standard elliptic regularity theory implies that for any $1<q<2, T(x) \in W_{l o c}^{2, q}\left(\mathbb{R}^{2}\right) \cap C^{\infty}\left(\mathbb{R}^{2} \backslash\{0\}\right)$, and the Sobolev embeddings yield that $T(x) \in W^{1,1 / \alpha}\left(B_{r}(0)\right) \cap C^{\alpha}\left(\overline{B_{r}(0)}\right)$ for any $r>0$ and $0<\alpha<1$.

Lemma $2.1([5])$. Let $T(x)$ be the function described in (2.1). There exists a function $H_{1}(x, y)$ such that

(i) for every $x, y \in \bar{\Omega}$,

$$
H(x, y)=H_{1}(x, y)+ \begin{cases}\frac{1}{2 \pi} \nabla \log a(y) \cdot T(x-y), & y \in \Omega, \\ \frac{1}{\pi} \nabla \log a(y) \cdot T(x-y), & y \in \partial \Omega,\end{cases}
$$

(ii) the mapping $y \in \bar{\Omega} \mapsto H_{1}(\cdot, y)$ belongs to $C^{1}\left(\Omega, C^{1}(\bar{\Omega})\right) \cap C^{1}\left(\partial \Omega, C^{1}(\bar{\Omega})\right)$.

In this way, $y \in \bar{\Omega} \mapsto H(\cdot, y) \in C\left(\Omega, C^{\alpha}(\bar{\Omega})\right) \cap C\left(\partial \Omega, C^{\alpha}(\bar{\Omega})\right)$ and $H(x, y) \in C^{\alpha}(\bar{\Omega} \times \Omega) \cap C^{\alpha}(\bar{\Omega} \times \partial \Omega) \cap C^{1}(\bar{\Omega} \times \Omega \backslash\{x=$ $y\}) \cap C^{1}(\bar{\Omega} \times \partial \Omega \backslash\{x=y\})$ for any $\alpha \in(0,1)$, and the corresponding Robin's function $y \in \bar{\Omega} \mapsto H(y, y)$ belongs to $C^{1}(\Omega) \cap C^{1}(\partial \Omega)$.

Let $d>0$ be a sufficiently small but fixed number such that for any $y \in \Omega$ with $\operatorname{dist}(y, \partial \Omega)<d$, we can define a reflection of $y$ across $\partial \Omega$ along the outer normal direction, $y^{*} \in \Omega^{c}$, and get that $\left|y-y^{*}\right|=2 \operatorname{dist}(y, \partial \Omega)$. Set

$$
\Omega_{d}:=\{y \in \Omega \mid \operatorname{dist}(y, \partial \Omega)<d\} .
$$

Lemma 2.2 ([5]). There exists a mapping $y \in \Omega_{d} \mapsto \mathrm{z}(\cdot, y) \in C\left(\Omega_{d}, C^{\alpha}(\bar{\Omega})\right) \cap L^{\infty}\left(\Omega_{d}, C^{\alpha}(\bar{\Omega})\right)$ for any $\alpha \in(0,1)$ such that for any $x \in \bar{\Omega}$ and $y \in \Omega_{d}$,

$$
H(x, y)=\frac{1}{2 \pi} \log \frac{1}{\left|x-y^{*}\right|}+z(x, y) .
$$

Even more, for any $x \in \bar{\Omega}$ and $y \in \Omega_{d}$,

$$
\mathrm{z}(x, y)=\frac{1}{2 \pi} \nabla \log a(y) \cdot T(x-y)-\frac{1}{2 \pi} \nabla \log a\left(y^{*}\right) \cdot T\left(x-y^{*}\right)+\tilde{z}(x, y),
$$

where the mapping $y \in \overline{\Omega_{d}} \mapsto \tilde{\mathrm{z}}(\cdot, y)$ belongs to $C^{1}\left(\overline{\Omega_{d}}, C^{1}(\bar{\Omega})\right)$.

Corollary 2.3. Under the assumptions in Lemma 2.2, the Robin's function $y \in \Omega \mapsto H(y, y)$ satisfies

$$
H(y, y)=\frac{1}{2 \pi} \log \frac{1}{\left|y-y^{*}\right|}+\mathrm{z}(y), \quad \forall y \in \Omega_{d}
$$

where $\mathrm{z} \in C^{1}\left(\overline{\Omega_{d}}\right)$ and

$$
\mathrm{z}(y)=\frac{1}{2 \pi} \nabla \log a(y) \cdot T(0)-\frac{1}{2 \pi} \nabla \log a\left(y^{*}\right) \cdot T\left(y-y^{*}\right)+\tilde{z}(y, y), \quad \forall y \in \Omega_{d} .
$$

The key ingredient to describe the shape of the approximate solution of (1.5) is based on the standard bubble

$$
U_{\delta, \xi}(x)=\log \frac{8 \delta^{2}}{\left(\delta^{2}+|x-\xi|^{2}\right)^{2}}, \quad \delta>0, \quad \xi \in \mathbb{R}^{2} .
$$


It is well known from [9] that these are all the solutions of the problem

$$
\left\{\begin{array}{l}
-\Delta u=e^{u} \quad \text { in } \mathbb{R}^{2} \\
\int_{\mathbb{R}^{2}} e^{u}<+\infty
\end{array}\right.
$$

The configuration space for $m$ concentration points $\xi=\left(\xi_{1}, \ldots, \xi_{m}\right)$ we try to seek is the following

$$
\mathcal{O}_{p}:=\left\{\xi=\left(\xi_{1}, \ldots, \xi_{m}\right) \in \Omega^{l} \times(\partial \Omega)^{m-l}\left|\min _{i, j=1, \ldots, m, i \neq j}\right| \xi_{i}-\xi_{j} \mid>\frac{1}{p^{\kappa}}, \min _{1 \leq i \leq l} \operatorname{dist}\left(\xi_{i}, \partial \Omega\right)>\frac{1}{p^{\kappa}}\right\},
$$

where $l \in\{0, \ldots, m\}$ and $\kappa$ is given by

$$
\kappa=2\left(m^{2}+1\right) .
$$

Let $m \in \mathbb{N}^{*}$ and $\xi=\left(\xi_{1}, \ldots, \xi_{m}\right) \in \mathcal{O}_{p}$ be fixed. Given number $\mu_{i}>0, i=1, \ldots, m$, yet to be chosen, we define

$$
\gamma=p^{\frac{p}{p-1}} e^{-\frac{p}{2(p-1)}}, \quad \varepsilon=e^{-\frac{1}{4} p}, \quad \delta_{i}=\varepsilon \mu_{i},
$$

and

$$
U_{i}(x)=\frac{1}{\gamma \mu_{i}^{2 /(p-1)}}\left[U_{\delta_{i}, \xi_{i}}(x)+\frac{1}{p} \omega_{1}\left(\frac{x-\xi_{i}}{\delta_{i}}\right)+\frac{1}{p^{2}} \omega_{2}\left(\frac{x-\xi_{i}}{\delta_{i}}\right)\right] .
$$

Here, $\omega_{j}, j=1,2$, are radial solutions of

$$
\Delta \omega_{j}+\frac{8}{\left(1+|z|^{2}\right)^{2}} \omega_{j}=\frac{8}{\left(1+|z|^{2}\right)^{2}} f_{j}(|z|) \quad \text { in } \quad \mathbb{R}^{2},
$$

with

having asymptotic

$$
f_{1}=\frac{1}{2} U_{1,0}^{2}, \quad f_{2}=\omega_{1} U_{1,0}-\frac{1}{3} U_{1,0}^{3}-\frac{1}{2} \omega_{1}^{2}-\frac{1}{8} U_{1,0}^{4}+\frac{1}{2} \omega_{1} U_{1,0}^{2},
$$

$$
\omega_{j}(r)=\frac{C_{j}}{2} \log \left(1+r^{2}\right)+O\left(\frac{1}{1+r}\right), \quad \partial_{r} \omega_{j}(r)=\frac{C_{j} r}{1+r^{2}}+O\left(\frac{1}{1+r^{2}}\right) \quad \text { as } r \rightarrow+\infty, r=|z|,
$$

where

$$
C_{j}=8 \int_{0}^{\infty} t \frac{t^{2}-1}{\left(t^{2}+1\right)^{3}} f_{j}(t) d t
$$

in particular,

$$
\begin{aligned}
\omega_{1}(z)= & \frac{1}{2} U_{1,0}^{2}(z)+6 \log \left(|z|^{2}+1\right)+\frac{2 \log 8-10}{|z|^{2}+1}+\frac{|z|^{2}-1}{|z|^{2}+1} \times\left\{-\frac{1}{2} \log ^{2} 8+2 \log ^{2}\left(|z|^{2}+1\right)\right. \\
& \left.+4 \int_{|z|^{2}}^{\infty} \frac{d s}{s+1} \log \frac{s+1}{s}-8 \log |z| \log \left(|z|^{2}+1\right)\right\},
\end{aligned}
$$

and

$$
C_{1}=12-4 \log 8
$$

(see [8, 22]). We now approximate the solution of problem (1.5) by

$$
U_{\xi}(x):=\sum_{i=1}^{m} P U_{i}(x)=\sum_{i=1}^{m}\left[U_{i}(x)+H_{i}(x)\right],
$$

where $H_{i}$ is a correction term defined as the solution of

$$
\begin{cases}-\Delta_{a} H_{i}+H_{i}=\nabla \log a(x) \nabla U_{i}-U_{i} & \text { in } \Omega, \\ \frac{\partial H_{i}}{\partial \nu}=-\frac{\partial U_{i}}{\partial \nu} & \text { on } \partial \Omega .\end{cases}
$$


In order to understand the asymptotic behavior of the correction term $H_{i}$, let us first use the convention

$$
c_{i}= \begin{cases}8 \pi, & \text { if } \quad \xi_{i} \in \Omega, \\ 4 \pi, & \text { if } \quad \xi_{i} \in \partial \Omega .\end{cases}
$$

Furthermore, we have the following result, whose proof is postponed to the Appendix.

Lemma 2.4. For any $0<\alpha<1$ and $\xi=\left(\xi_{1}, \ldots, \xi_{m}\right) \in \mathcal{O}_{p}$, then we have

$$
H_{i}(x)=\frac{1}{\gamma \mu_{i}^{2 /(p-1)}}\left[\left(1-\frac{C_{1}}{4 p}-\frac{C_{2}}{4 p^{2}}\right) c_{i} H\left(x, \xi_{i}\right)-\log \left(8 \delta_{i}^{2}\right)+\left(\frac{C_{1}}{p}+\frac{C_{2}}{p^{2}}\right) \log \delta_{i}+O\left(\delta_{i}^{\alpha / 2}\right)\right]
$$

uniformly in $\bar{\Omega}$, where $H$ is the regular part of Green's function defined in (1.7).

From Lemma 2.4 we have that away from each point $\xi_{i}$, namely $\left|x-\xi_{i}\right| \geq 1 / p^{2 \kappa}$ for any $i=1, \ldots, m$,

$$
U_{\xi}(x)=\sum_{i=1}^{m} \frac{1}{\gamma \mu_{i}^{2 /(p-1)}}\left[\left(1-\frac{C_{1}}{4 p}-\frac{C_{2}}{4 p^{2}}\right) c_{i} G\left(x, \xi_{i}\right)+\frac{1}{p} O\left(\delta_{i}^{\alpha / 2}\right)\right] .
$$

While for $\left|x-\xi_{i}\right|<1 / p^{2 \kappa}$ with some $i$, by (2.6), (2.10), (2.13), (2.19) and the fact that $H\left(\cdot, \xi_{k}\right) \in C^{\alpha}(\bar{\Omega})$ for any $\xi_{k} \in \bar{\Omega}$ and any $\alpha \in(0,1)$ we obtain

$$
\begin{aligned}
P U_{i}(x)= & \frac{1}{\gamma \mu_{i}^{2 /(p-1)}}\left[U_{1,0}\left(\frac{x-\xi_{i}}{\delta_{i}}\right)+\frac{1}{p} \omega_{1}\left(\frac{x-\xi_{i}}{\delta_{i}}\right)+\frac{1}{p^{2}} \omega_{2}\left(\frac{x-\xi_{i}}{\delta_{i}}\right)+\left(1-\frac{C_{1}}{4 p}-\frac{C_{2}}{4 p^{2}}\right) c_{i} H\left(\xi_{i}, \xi_{i}\right)\right. \\
& \left.-\log \left(8 \delta_{i}^{4}\right)+\left(\frac{C_{1}}{p}+\frac{C_{2}}{p^{2}}\right) \log \delta_{i}+O\left(\left|x-\xi_{i}\right|^{\alpha}+\delta_{i}^{\alpha / 2}\right)\right],
\end{aligned}
$$

and for any $k \neq i$,

$$
\begin{aligned}
P U_{k}(x)= & \frac{1}{\gamma \mu_{k}^{2 /(p-1)}}\left[\log \frac{8 \delta_{k}^{2}}{\left(\delta_{k}^{2}+\left|x-\xi_{k}\right|^{2}\right)^{2}}+\frac{1}{p} \omega_{1}\left(\frac{x-\xi_{k}}{\delta_{k}}\right)+\frac{1}{p^{2}} \omega_{2}\left(\frac{x-\xi_{k}}{\delta_{k}}\right)\right. \\
& \left.+\left(1-\frac{C_{1}}{4 p}-\frac{C_{2}}{4 p^{2}}\right) c_{k} H\left(x, \xi_{k}\right)-\log \left(8 \delta_{k}^{2}\right)+\left(\frac{C_{1}}{p}+\frac{C_{2}}{p^{2}}\right) \log \delta_{k}+O\left(\delta_{k}^{\alpha / 2}\right)\right] \\
= & \frac{1}{\gamma \mu_{k}^{2 /(p-1)}}\left[\left(1-\frac{C_{1}}{4 p}-\frac{C_{2}}{4 p^{2}}\right) c_{k} G\left(\xi_{i}, \xi_{k}\right)+O\left(\left|x-\xi_{i}\right|^{\alpha}+\delta_{k}^{\alpha / 2}\right)\right] .
\end{aligned}
$$

Hence for $\left|x-\xi_{i}\right|<1 / p^{2 \kappa}$,

$$
U_{\xi}(x)=\frac{1}{\gamma \mu_{i}^{2 /(p-1)}}\left[p+U_{1,0}\left(\frac{x-\xi_{i}}{\delta_{i}}\right)+\frac{1}{p} \omega_{1}\left(\frac{x-\xi_{i}}{\delta_{i}}\right)+\frac{1}{p^{2}} \omega_{2}\left(\frac{x-\xi_{i}}{\delta_{i}}\right)+O\left(\left|x-\xi_{i}\right|^{\alpha}+\sum_{k=1}^{m} \delta_{k}^{\alpha / 2}\right)\right]
$$

is a good approximation for a solution of problem (1.5) provided that the concentration parameters $\mu_{i}, i=1, \ldots, m$, are the solution of the nonlinear system

$$
\log \left(8 \mu_{i}^{4}\right)=\left(1-\frac{C_{1}}{4 p}-\frac{C_{2}}{4 p^{2}}\right) c_{i} H\left(\xi_{i}, \xi_{i}\right)+\left(\frac{C_{1}}{p}+\frac{C_{2}}{p^{2}}\right) \log \delta_{i}+\left(1-\frac{C_{1}}{4 p}-\frac{C_{2}}{4 p^{2}}\right) \sum_{k \neq i}^{m}\left(\frac{\mu_{i}}{\mu_{k}}\right)^{2 /(p-1)} c_{k} G\left(\xi_{i}, \xi_{k}\right) .
$$

Indeed, the parameters $\mu=\left(\mu_{1}, \ldots, \mu_{m}\right)$ are well defined in system $(2.22)$, which is stated as follows and proved in the Appendix.

Lemma 2.5. For any points $\xi=\left(\xi_{1}, \ldots, \xi_{m}\right) \in \mathcal{O}_{p}$ and any $p>1$ large enough, system (2.22) has a unique solution $\mu=\left(\mu_{1}, \ldots, \mu_{m}\right)$ satisfying

$$
1 / C \leq \mu_{i} \leq C p^{\kappa} \quad \forall i=1, \ldots, m,
$$


for some $C>0$. Moreover, for any $i=1, \ldots, m$, one has

$$
\left|D_{\xi} \log \mu_{i}\right| \leq C p^{\kappa}
$$

and

$$
\mu_{i}=e^{-\frac{3}{4}+\frac{1}{4} c_{i} H\left(\xi_{i}, \xi_{i}\right)+\frac{1}{4} \sum_{k=1, k \neq i}^{m} c_{k} G\left(\xi_{i}, \xi_{k}\right)}\left[1+O\left(\frac{\log ^{2} p}{p}\right)\right]
$$

Remark 2.6. Observe that for $\left|x-\xi_{i}\right|=\delta_{i}|z|<1 / p^{2 \kappa}$, by (2.6), (2.9), (2.13) and (2.23),

$$
p+U_{1,0}(z)+\frac{1}{p} \omega_{1}(z)+\frac{1}{p^{2}} \omega_{2}(z) \geq p-2 \log \left(1+|z|^{2}\right)+O(1) \geq 8 \kappa \log p+4 \log \mu_{i}+O(1)>7 \kappa \log p .
$$

Hence by (2.21), we can easily get that $0<U_{\xi} \leq 2 \sqrt{e}$ in $B_{1 / p^{2 \kappa}}\left(\xi_{i}\right)$, and $\sup _{B_{1 / p^{2 \kappa}\left(\xi_{i}\right)}} U_{\xi} \rightarrow \sqrt{e}$ as $p \rightarrow+\infty$. Moreover, by the maximum principle, we see that $G\left(x, \xi_{i}\right)>0$ over $\bar{\Omega}$ and thus by (2.20), $U_{\xi}$ is a positive, uniformly bounded function over $\bar{\Omega}$. In conclusion, $0<U_{\xi} \leq 2 \sqrt{e}$ over $\bar{\Omega}$.

Let us perform the change of variables

$$
v(y)=\varepsilon^{2 /(p-1)} u(\varepsilon y), \quad \forall y \in \Omega_{p}:=e^{p / 4} \Omega .
$$

Then by the definition of $\varepsilon$ in (2.9), $u(x)$ solves equation (1.5) if and only if the function $v(y)$ satisfies

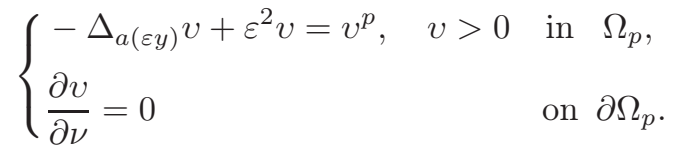

We write $\xi_{i}^{\prime}=\xi_{i} / \varepsilon, i=1, \ldots, m$ and define the initial approximate solution of (2.26) as

$$
V_{\xi^{\prime}}(y)=\varepsilon^{2 /(p-1)} U_{\xi}(\varepsilon y),
$$

with $\xi^{\prime}=\left(\xi_{1}^{\prime}, \ldots, \xi_{m}^{\prime}\right)$ and $U_{\xi}$ defined in (2.16). Let us set

$$
S_{p}(v)=-\Delta_{a(\varepsilon y)} v+\varepsilon^{2} v-v_{+}^{p}, \quad \text { where } v_{+}=\max \{v, 0\},
$$

and introduce the functional

$$
I_{p}(v)=\frac{1}{2} \int_{\Omega_{p}} a(\varepsilon y)\left(|\nabla v|^{2}+\varepsilon^{2} v^{2}\right) d y-\frac{1}{p+1} \int_{\Omega_{p}} a(\varepsilon y) v_{+}^{p+1} d y, \quad v \in H^{1}\left(\Omega_{p}\right),
$$

whose nontrivial critical points are solutions of problem (2.26). In fact, by the maximum principle, problem (2.26) is equivalent to

$$
S_{p}(v)=0, \quad v_{+} \not \equiv 0 \quad \text { in } \Omega_{p}, \quad \frac{\partial v}{\partial \nu}=0 \quad \text { on } \partial \Omega_{p} .
$$

We will look for solutions of problem (2.26) in the form $v=V_{\xi^{\prime}}+\phi$, where $\phi$ will represent a higher-order correction in the expansion of $v$. Observe that

$$
S_{p}\left(V_{\xi^{\prime}}+\phi\right)=\mathcal{L}(\phi)+R_{\xi^{\prime}}+N(\phi)=0,
$$

where

$$
\mathcal{L}(\phi)=-\Delta_{a(\varepsilon y)} \phi+\varepsilon^{2} \phi-W_{\xi^{\prime}} \phi \quad \text { with } \quad W_{\xi^{\prime}}=p V_{\xi^{\prime}}^{p-1},
$$

and

$$
R_{\xi^{\prime}}=-\Delta_{a(\varepsilon y)} V_{\xi^{\prime}}+\varepsilon^{2} V_{\xi^{\prime}}-V_{\xi^{\prime}}^{p}, \quad N(\phi)=-\left[\left(V_{\xi^{\prime}}+\phi\right)_{+}^{p}-V_{\xi^{\prime}}^{p}-p V_{\xi^{\prime}}^{p-1} \phi\right] .
$$

In terms of $\phi$, problem (2.26) becomes

$$
\begin{cases}\mathcal{L}(\phi)=-\left[R_{\xi^{\prime}}+N(\phi)\right] & \text { in } \quad \Omega_{p}, \\ \frac{\partial \phi}{\partial \nu}=0 & \text { on } \partial \Omega_{p} .\end{cases}
$$


For any $\xi=\left(\xi_{1}, \ldots, \xi_{m}\right) \in \mathcal{O}_{p}$ and $h \in L^{\infty}\left(\Omega_{p}\right)$, let us introduce a weighted $L^{\infty}$-norm defined as

$$
\|h\|_{*}=\sup _{y \in \Omega_{p}}\left|\left(\sum_{i=1}^{m} \frac{\mu_{i}^{\sigma}}{\left(\mu_{i}+\left|y-\xi_{i}^{\prime}\right|\right)^{2+\sigma}}+\varepsilon^{2}\right)^{-1} h(y)\right|,
$$

where $\sigma>0$ is small but fixed independent of $p$. With respect to the $\|\cdot\|_{*}$-norm, the error term $R_{\xi^{\prime}}$ defined in $(2.28)$ can be estimated as follows.

Proposition 2.7. Let $m$ be a positive integer. There exist constants $C>0$ and $p_{m}>1$ such that for any $\xi=$ $\left(\xi_{1}, \ldots, \xi_{m}\right) \in \mathcal{O}_{p}$ and any $p>p_{m}$,

$$
\left\|R_{\xi^{\prime}}\right\|_{*} \leq \frac{C}{p^{4}}
$$

Proof. Observe that, by (2.16), (2.17) and (2.27),

$$
-\Delta_{a(\varepsilon y)} V_{\xi^{\prime}}+\varepsilon^{2} V_{\xi^{\prime}}=\varepsilon^{2} \sum_{i=1}^{m} \varepsilon^{2 /(p-1)}\left[-\Delta_{a}\left(U_{i}+H_{i}\right)+\left(U_{i}+H_{i}\right)\right]=-\varepsilon^{2 p /(p-1)} \sum_{i=1}^{m} \Delta U_{i} .
$$

Then by (2.6), (2.9), (2.10) and (2.11),

$$
-\Delta_{a(\varepsilon y)} V_{\xi^{\prime}}+\varepsilon^{2} V_{\xi^{\prime}}=\sum_{i=1}^{m} \frac{1}{p^{p /(p-1)} \mu_{i}^{2 p /(p-1)}} e^{U_{1,0}(z)}\left[1-\frac{1}{p} f_{1}(z)-\frac{1}{p^{2}} f_{2}(z)+\frac{1}{p} \omega_{1}(z)+\frac{1}{p^{2}} \omega_{2}(z)\right]
$$

with $z=\left(\varepsilon y-\xi_{i}\right) / \delta_{i}$. By (2.6), (2.13) and (2.23) we get, if $\left|\varepsilon y-\xi_{i}\right|=\delta_{i}|z| \geq 1 / p^{2 \kappa}$ for any $i=1, \ldots, m$, then

$$
U_{1,0}(z)=-p+O(\log p), \quad \omega_{j}(z)=\frac{1}{4} C_{j} p+O(\log p), \quad j=1,2,
$$

and hence, by (2.12) and (2.32),

$$
-\Delta_{a(\varepsilon y)} V_{\xi^{\prime}}+\varepsilon^{2} V_{\xi^{\prime}}=\sum_{i=1}^{m} \frac{e^{U_{1,0}(z)}}{p^{p /(p-1)} \mu_{i}^{2 p /(p-1)}} O\left(p^{2}\right) .
$$

On the other hand, in the same region, by (2.20) and (2.27) we get

$$
V_{\xi^{\prime}}^{p}=O\left(\frac{1}{p}\left(\frac{\log p}{p}\right)^{p}\right)
$$

which, together with (2.9), (2.23) and (2.33), implies

$$
\left|\left(\sum_{i=1}^{m} \frac{\mu_{i}^{\sigma}}{\left(\mu_{i}+\left|y-\xi_{i}^{\prime}\right|\right)^{2+\sigma}}+\varepsilon^{2}\right)^{-1} R_{\xi^{\prime}}(y)\right| \leq C\left[p \sum_{i=1}^{m}\left|\frac{y-\xi_{i}^{\prime}}{\mu_{i}}\right|^{\sigma-2}+\frac{1}{p}\left(\frac{\sqrt{e} \log p}{p}\right)^{p}\right]=o\left(e^{-p / 4}\right) .
$$

Let us fix an index $i \in\{1, \ldots, m\}$ and the region $\left|\varepsilon y-\xi_{i}\right|=\delta_{i}|z|<1 / p^{2 \kappa}$. By (2.21), (2.27) and the relation

$$
\left(\frac{p \varepsilon^{2 /(p-1)}}{\gamma \mu_{i}^{2 /(p-1)}}\right)^{p}=\frac{1}{p^{p /(p-1)} \mu_{i}^{2 p /(p-1)}},
$$

we get, for $\left|\varepsilon y-\xi_{i}\right|=\delta_{i}|z|<1 / p^{2 \kappa}$,

$$
V_{\xi^{\prime}}^{p}(y)=\frac{1}{p^{p /(p-1)} \mu_{i}^{2 p /(p-1)}}\left\{1+\frac{U_{1,0}(z)}{p}+\frac{\omega_{1}(z)}{p^{2}}+\frac{1}{p^{3}}\left[\omega_{2}(z)+O\left(p^{2} \delta_{i}^{\alpha}|z|^{\alpha}+p^{2} \sum_{k=1}^{m} \delta_{k}^{\alpha / 2}\right)\right]\right\}^{p} .
$$


From a Taylor expansion of the exponential and logarithmic functions

$$
\begin{aligned}
\left(1+\frac{a}{p}+\frac{b}{p^{2}}+\frac{c}{p^{3}}\right)^{p}= & e^{a}\left[1+\frac{1}{p}\left(b-\frac{a^{2}}{2}\right)+\frac{1}{p^{2}}\left(c-a b+\frac{a^{3}}{3}+\frac{b^{2}}{2}-\frac{a^{2} b}{2}+\frac{a^{4}}{8}\right)\right. \\
& \left.+O\left(\frac{\log ^{6}(|z|+2)}{p^{3}}\right)\right],
\end{aligned}
$$

which holds for $|z| \leq C e^{p / 8}$ provided $-4 \log (|z|+2) \leq a(z) \leq C$ and $|b(z)|+|c(z)| \leq C \log (|z|+2)$, so we have that for $\left|\varepsilon y-\xi_{i}\right|=\delta_{i}|z| \leq \sqrt{\delta_{i}} / p^{2 \kappa}$,

$$
\begin{aligned}
V_{\xi^{\prime}}^{p}(y)= & \frac{1}{p^{p /(p-1)} \mu_{i}^{2 p /(p-1)}} e^{U_{1,0}(z)}\left[1+\frac{1}{p}\left(\omega_{1}-\frac{1}{2} U_{1,0}^{2}\right)(z)+\frac{1}{p^{2}}\left(\omega_{2}-\omega_{1} U_{1,0}+\frac{1}{3} U_{1,0}^{3}+\frac{1}{2} \omega_{1}^{2}\right.\right. \\
& \left.\left.-\frac{1}{2} \omega_{1} U_{1,0}^{2}+\frac{1}{8} U_{1,0}^{4}\right)(z)+O\left(\frac{\log ^{6}(|z|+2)}{p^{3}}+\delta_{i}^{\alpha}|z|^{\alpha}+\sum_{k=1}^{m} \delta_{k}^{\alpha / 2}\right)\right],
\end{aligned}
$$

which combined with (2.12) and (2.32) gives

$$
R_{\xi^{\prime}}(y)=-\Delta_{a(\varepsilon y)} V_{\xi^{\prime}}+\varepsilon^{2} V_{\xi^{\prime}}-V_{\xi^{\prime}}^{p}=\frac{1}{p^{p /(p-1)} \mu_{i}^{2 p /(p-1)}} e^{U_{1,0}(z)} O\left(\frac{\log ^{6}(|z|+2)}{p^{3}}+\delta_{i}^{\alpha}|z|^{\alpha}+\sum_{k=1}^{m} \delta_{k}^{\alpha / 4}\right) .
$$

Hence, in this region we get

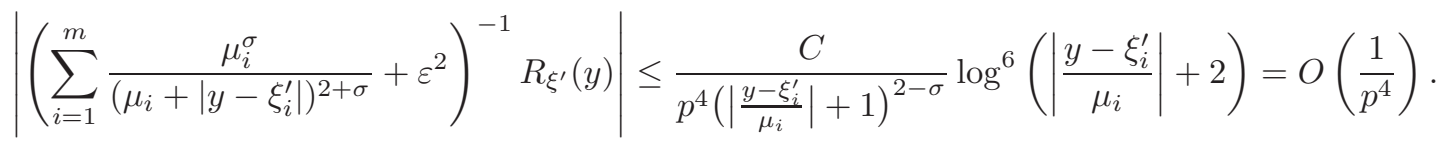

Finally, in the remaining region $\sqrt{\delta_{i}} / p^{2 \kappa}<\left|\varepsilon y-\xi_{i}\right|=\delta_{i}|z|<1 / p^{2 \kappa}$, we have that, by (2.12) and (2.32),

$$
-\Delta_{a(\varepsilon y)} V_{\xi^{\prime}}+\varepsilon^{2} V_{\xi^{\prime}}=\frac{e^{U_{1,0}(z)}}{p^{p /(p-1)} \mu_{i}^{2 p /(p-1)}} O\left(p^{2}\right),
$$

and by $(2.36)$,

$$
V_{\xi^{\prime}}^{p}(y)=\frac{e^{U_{1,0}(z)}}{p^{p /(p-1)} \mu_{i}^{2 p /(p-1)}} O(1),
$$

since $\left(1+\frac{s}{p}\right)^{p} \leq e^{s}$. Thus, in this region,

$$
\left|\left(\sum_{i=1}^{m} \frac{\mu_{i}^{\sigma}}{\left(\mu_{i}+\left|y-\xi_{i}^{\prime}\right|\right)^{2+\sigma}}+\varepsilon^{2}\right)^{-1} R_{\xi^{\prime}}(y)\right| \leq C p\left|\frac{y-\xi_{i}^{\prime}}{\mu_{i}}\right|^{\sigma-2}=o\left(e^{-p / 8}\right) .
$$

Combining $(2.30),(2.34),(2.38)$ with $(2.39)$, we conclude that estimate (2.31) holds.

\section{ANALYSIS OF THE LINEARIZED OPERATOR}

In this section, we prove bounded invertibility of the operator $\mathcal{L}$, uniformly on $\xi \in \mathcal{O}_{p}$, by using the weighted $L^{\infty}$-norm defined in (2.30). Let us recall that $\mathcal{L}(\phi)=-\Delta_{a(\varepsilon y)} \phi+\varepsilon^{2} \phi-W_{\xi^{\prime}} \phi$, where $W_{\xi^{\prime}}=p V_{\xi^{\prime}}^{p-1}$. As in Proposition 2.7 , we have the following expansions with respect to the potential $W_{\xi^{\prime}}$.

Lemma 3.1. Let $m$ be a positive integer. There exist constants $D_{0}>0$ and $p_{m}>1$ such that

$$
W_{\xi^{\prime}}(y) \leq D_{0} \sum_{i=1}^{m} \frac{1}{\mu_{i}^{2}} e^{U_{1,0}\left(\frac{y-\xi_{i}^{\prime}}{\mu_{i}}\right)}
$$


for any points $\xi=\left(\xi_{1}, \ldots, \xi_{m}\right) \in \mathcal{O}_{p}$ and any $p \geq p_{m}$. Furthermore,

$$
W_{\xi^{\prime}}(y)=\frac{8}{\mu_{i}^{2}\left(1+|z|^{2}\right)^{2}}\left[1+\frac{1}{p}\left(\omega_{1}-U_{1,0}-\frac{1}{2} U_{1,0}^{2}\right)(z)+O\left(\frac{\log ^{4}(|z|+2)}{p^{2}}\right)\right]
$$

for any $\left|\varepsilon y-\xi_{i}\right| \leq \sqrt{\delta_{i}} / p^{2 \kappa}$, where $z=\left(y-\xi_{i}^{\prime}\right) / \mu_{i}$.

Proof. If $\left|\varepsilon y-\xi_{i}\right|=\delta_{i}|z|<1 / p^{2 \kappa}$ for some $i=1, \ldots, m$, by (2.21), (2.27) and (2.35),

$$
\begin{aligned}
W_{\xi^{\prime}}(y) & =p\left(\frac{\varepsilon^{2 /(p-1)}}{\gamma \mu_{i}^{2 /(p-1)}}\right)^{p-1}\left[p+U_{1,0}(z)+\frac{1}{p} \omega_{1}(z)+\frac{1}{p^{2}} \omega_{2}(z)+O\left(\delta_{i}^{\alpha}|z|^{\alpha}+\sum_{k=1}^{m} \delta_{k}^{\alpha / 2}\right)\right]^{p-1} \\
& =\frac{1}{\mu_{i}^{2}}\left[1+\frac{1}{p} U_{1,0}(z)+\frac{1}{p^{2}} \omega_{1}(z)+\frac{1}{p^{3}} \omega_{2}(z)+\frac{1}{p} O\left(\delta_{i}^{\alpha}|z|^{\alpha}+\sum_{k=1}^{m} \delta_{k}^{\alpha / 2}\right)\right]^{p-1} .
\end{aligned}
$$

In this region, using the fact that $(1+a / p)^{p-1} \leq e^{(p-1) a / p}$ and $U_{1,0}(z) \geq-p+O(\log p)$, we get

$$
W_{\xi^{\prime}}(y) \leq \frac{C}{\mu_{i}^{2}} e^{U_{1,0}(z)} e^{-U_{1,0}(z) / p}=O\left(\frac{1}{\mu_{i}^{2}} e^{U_{1,0}(z)}\right) .
$$

In particular, from a slight modification of formula (2.37), namely

$$
\left(1+\frac{a}{p}+\frac{b}{p^{2}}+\frac{c}{p^{3}}\right)^{p-1}=e^{a}\left[1+\frac{1}{p}\left(b-a-\frac{a^{2}}{2}\right)+O\left(\frac{\log ^{4}(|z|+2)}{p^{2}}\right)\right],
$$

we conclude that if $\left|\varepsilon y-\xi_{i}\right|=\delta_{i}|z| \leq \sqrt{\delta_{i}} / p^{2 \kappa}$, then

$$
W_{\xi^{\prime}}(y)=\frac{1}{\mu_{i}^{2}} e^{U_{1,0}(z)}\left[1+\frac{1}{p}\left(\omega_{1}-U_{1,0}-\frac{1}{2} U_{1,0}^{2}\right)(z)+O\left(\frac{\log ^{4}(|z|+2)}{p^{2}}\right)\right] .
$$

Additionally, if $\left|\varepsilon y-\xi_{i}\right|=\delta_{i}|z| \geq 1 / p^{2 \kappa}$ for all $i$, then by (2.9), (2.20), (2.23) and (2.27),

$$
W_{\xi^{\prime}}(y)=p \varepsilon^{2} U_{\xi}^{p-1}(\varepsilon y) \leq p \varepsilon^{2}\left(\frac{C \log p}{\gamma}\right)^{p-1}=O\left(\left(\frac{\log p}{p}\right)^{p-1}\right)=o\left(\frac{1}{\mu_{i}^{2}} e^{U_{1,0}(z)}\right) .
$$

Remark 3.2. As for $W_{\xi^{\prime}}$, we mention that if $\left|\varepsilon y-\xi_{i}\right|<1 / p^{2 \kappa}$ for some $i=1, \ldots, m$, then

$$
p\left[V_{\xi^{\prime}}(y)+O\left(\frac{1}{p^{3}}\right)\right]^{p-2} \leq C p\left(\frac{p \varepsilon^{2 /(p-1)}}{\gamma \mu_{i}^{2 /(p-1)}}\right)^{p-2} e^{\frac{p-2}{p} U_{1,0}\left(\frac{y-\xi_{i}^{\prime}}{\mu_{i}}\right)}=O\left(\frac{1}{\mu_{i}^{2}} e^{U_{1,0}\left(\frac{y-\xi_{i}^{\prime}}{\mu_{i}}\right)}\right) .
$$

Since this estimate is true if $\left|\varepsilon y-\xi_{i}\right| \geq 1 / p^{2 \kappa}$ for all $i$, we get

$$
p\left[V_{\xi^{\prime}}(y)+O\left(\frac{1}{p^{3}}\right)\right]^{p-2} \leq C \sum_{i=1}^{m} \frac{1}{\mu_{i}^{2}} e^{U_{1,0}\left(\frac{y-\xi_{i}^{\prime}}{\mu_{i}}\right)} .
$$

Let

$$
Z_{0}(z)=\frac{|z|^{2}-1}{|z|^{2}+1}, \quad Z_{j}(z)=\frac{z_{j}}{|z|^{2}+1}, \quad j=1,2 .
$$

It is well known (see $[6,9])$ that

- any bounded solution to

$$
\Delta \phi+\frac{8}{\left(1+|z|^{2}\right)^{2}} \phi=0 \quad \text { in } \mathbb{R}^{2},
$$

is a linear combination of $Z_{j}, j=0,1,2$; 
- any bounded solution to

$$
\Delta \phi+\frac{8}{\left(1+|z|^{2}\right)^{2}} \phi=0 \quad \text { in } \mathbb{R}_{+}^{2}, \quad \frac{\partial \phi}{\partial \nu}=0 \quad \text { on } \partial \mathbb{R}_{+}^{2},
$$

where $\mathbb{R}_{+}^{2}=\left\{\left(z_{1}, z_{2}\right): z_{2}>0\right\}$, is a linear combination of $Z_{j}, j=0,1$.

Now we consider the following linear problem: given $h \in C\left(\bar{\Omega}_{p}\right)$ and points $\xi=\left(\xi_{1}, \ldots, \xi_{m}\right) \in \mathcal{O}_{p}$, we find a function $\phi \in H^{2}\left(\Omega_{p}\right)$ and scalars $c_{i j} \in \mathbb{R}, i=1, \ldots, m, j=1, J_{i}$, such that

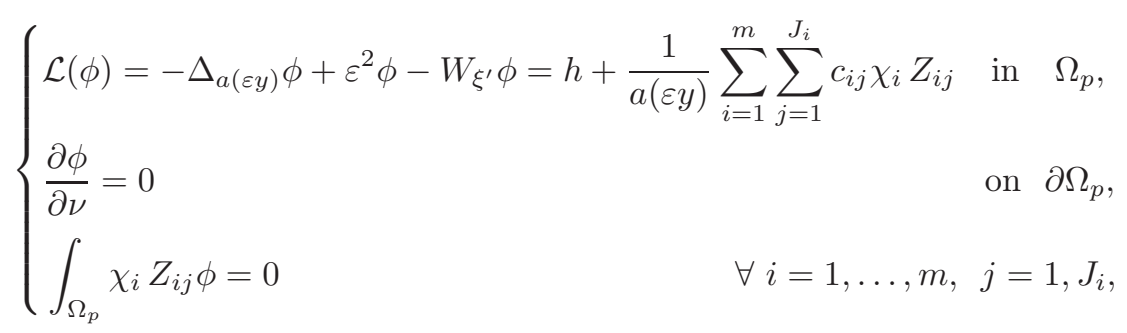

where $J_{i}=2$ if $i=1, \ldots, l$ while $J_{i}=1$ if $i=l+1, \ldots, m$, and $Z_{i j}, \chi_{i}$, are defined as follows.

Let $\chi: \mathbb{R} \rightarrow[0,1]$ be a smooth, non-increasing cut-off function such that for a large but fixed number $R_{0}>0$, $\chi(r)=1$ if $r \leq R_{0}$, and $\chi(r)=0$ if $r \geq R_{0}+1$.

For $i=1, \ldots, l$ (corresponding to interior spike case), we define

$$
\chi_{i}(y)=\chi\left(\frac{\left|y-\xi_{i}^{\prime}\right|}{\mu_{i}}\right), \quad Z_{i j}(y)=\frac{1}{\mu_{i}} Z_{j}\left(\frac{y-\xi_{i}^{\prime}}{\mu_{i}}\right), \quad j=0,1,2 .
$$

For $i=l+1, \ldots, m$ (corresponding to boundary spike case), we have to straighten the boundary first. More precisely, at the boundary point $\xi_{i} \in \partial \Omega$, we define a rotation map $A_{i}: \mathbb{R}^{2} \mapsto \mathbb{R}^{2}$ such that $A_{i} \nu_{\Omega}\left(\xi_{i}\right)=\nu_{\mathbb{R}_{+}^{2}}(0)$. Let $\mathcal{G}\left(x_{1}\right)$ be the defining function for the boundary $A_{i}\left(\partial \Omega-\left\{\xi_{i}\right\}\right)$ in a small neighborhood $B_{\delta}(0,0)$ of the origin, that is, there exist $R_{1}>0, \delta>0$ small and a smooth function $\mathcal{G}:\left(-R_{1}, R_{1}\right) \mapsto \mathbb{R}$ satisfying $\mathcal{G}(0)=0, \mathcal{G}^{\prime}(0)=0$ and such that $A_{i}\left(\Omega-\left\{\xi_{i}\right\}\right) \cap B_{\delta}(0,0)=\left\{\left(x_{1}, x_{2}\right):-R_{1}<x_{1}<R_{1}, x_{2}>\mathcal{G}\left(x_{1}\right)\right\} \cap B_{\delta}(0,0)$. Furthermore, we consider the flattening change of variables $F_{i}: B_{\delta}(0,0) \cap \overline{A_{i}\left(\Omega-\left\{\xi_{i}\right\}\right)} \mapsto \mathbb{R}^{2}$ be defined by $F_{i}=\left(F_{i 1}, F_{i 2}\right)$, where

$$
F_{i 1}=x_{1}+\frac{x_{2}-\mathcal{G}\left(x_{1}\right)}{1+\left|\mathcal{G}^{\prime}\left(x_{1}\right)\right|^{2}} \mathcal{G}^{\prime}\left(x_{1}\right) \quad \text { and } \quad F_{i 2}=x_{2}-\mathcal{G}\left(x_{1}\right) .
$$

Then for any $i=l+1, \ldots, m$, we set

$$
F_{i}^{p}(y)=e^{p / 4} F_{i}\left(A_{i}\left(e^{-p / 4} y-\xi_{i}\right)\right)=\frac{1}{\varepsilon} F_{i}\left(A_{i}\left(\varepsilon y-\xi_{i}\right)\right),
$$

and define

$$
\chi_{i}(y)=\chi\left(\frac{1}{\mu_{i}}\left|F_{i}^{p}(y)\right|\right), \quad Z_{i j}(y)=\frac{1}{\mu_{i}} Z_{j}\left(\frac{1}{\mu_{i}} F_{i}^{p}(y)\right), \quad j=0,1 .
$$

It is important to note that $F_{i}^{p}, i=l+1, \ldots, m$, preserves the Neumann boundary condition and

$$
\Delta_{a(\varepsilon y)} Z_{i 0}+\frac{8 \mu_{i}^{2}}{\left(\mu_{i}^{2}+\left|y-\xi_{i}^{\prime}\right|^{2}\right)^{2}} Z_{i 0}=O\left(\frac{\varepsilon \mu_{i}}{\left(\mu_{i}+\left|y-\xi_{i}^{\prime}\right|\right)^{3}}\right), \quad \forall i=1, \ldots, m .
$$

Proposition 3.3. Let $m$ be a positive integer. Then there exist constants $C>0$ and $p_{m}>1$ such that for any $p>p_{m}$, any points $\xi=\left(\xi_{1}, \ldots, \xi_{m}\right) \in \mathcal{O}_{p}$ and any $h \in C\left(\bar{\Omega}_{p}\right)$, there is a unique solution $\phi \in H^{2}\left(\Omega_{p}\right)$ of problem (3.6) for some coefficients $c_{i j} \in \mathbb{R}, i=1, \ldots, m, j=1, J_{i}$, which satisfies

$$
\|\phi\|_{L^{\infty}\left(\Omega_{p}\right)} \leq C p\|h\|_{*} .
$$

The proof of this result will be split into four steps which we state and prove next.

Step 1: Constructing a suitable barrier. 
Lemma 3.4. There exist constants $R_{1}>0$ and $C>0$, independent of $p$, such that for any sufficiently large $p$, any points $\xi=\left(\xi_{1}, \ldots, \xi_{m}\right) \in \mathcal{O}_{p}$ and any $\sigma \in(0,1)$, there is a function

$$
\psi: \Omega_{p} \backslash \bigcup_{i=1}^{m} B_{R_{1} \mu_{i}}\left(\xi_{i}^{\prime}\right) \mapsto \mathbb{R}
$$

smooth and positive so that

$$
\begin{aligned}
& \mathcal{L}(\psi)=-\Delta_{a(\varepsilon y)} \psi+\varepsilon^{2} \psi-W_{\xi^{\prime}} \psi \geq \sum_{i=1}^{m} \frac{\mu_{i}^{\sigma}}{\left|y-\xi_{i}^{\prime}\right|^{2+\sigma}}+\varepsilon^{2} \quad \text { in } \quad \Omega_{p} \backslash \bigcup_{i=1}^{m} B_{R_{1} \mu_{i}}\left(\xi_{i}^{\prime}\right), \\
& \frac{\partial \psi}{\partial \nu} \geq 0 \quad \text { on } \partial \Omega_{p} \backslash \bigcup_{i=1}^{m} B_{R_{1} \mu_{i}}\left(\xi_{i}^{\prime}\right) \text {, } \\
& \psi>0 \\
& \text { in } \quad \Omega_{p} \backslash \bigcup_{i=1}^{m} B_{R_{1} \mu_{i}}\left(\xi_{i}^{\prime}\right) \\
& \psi \geq 1 \\
& \text { on } \Omega_{p} \cap\left(\bigcup_{i=1}^{m} \partial B_{R_{1} \mu_{i}}\left(\xi_{i}^{\prime}\right)\right) \text {. }
\end{aligned}
$$

Moreover, $\psi$ is uniformly bounded, i.e.

$$
1<\psi \leq C \quad \text { in } \Omega_{p} \backslash \bigcup_{i=1}^{m} B_{R_{1} \mu_{i}}\left(\xi_{i}^{\prime}\right) .
$$

Proof. Let us take

$$
\psi=\sum_{i=1}^{m}\left(1-\frac{\mu_{i}^{\sigma}}{\left|y-\xi_{i}^{\prime}\right|^{\sigma}}\right)+C_{1} \Psi_{0}(y)
$$

where $\Psi_{0}$ is the unique solution of

$$
\begin{cases}-\Delta_{a(\varepsilon y)} \Psi_{0}+\varepsilon^{2} \Psi_{0}=\varepsilon^{2} & \text { in } \Omega_{p} \\ \frac{\partial \Psi_{0}}{\partial \nu}=\varepsilon & \text { on } \partial \Omega_{p} .\end{cases}
$$

Observing that $\Psi_{0}$ is uniformly bounded in $\Omega_{p}$, it is directly checked that, choosing the positive constant $C_{1}$ larger if necessary, $\psi$ satisfies all the properties of the lemma for large enough numbers $R_{1}$ and $p$.

Step 2: An auxiliary linear equation. Given $h \in C^{0, \alpha}\left(\bar{\Omega}_{p}\right)$ and $\xi=\left(\xi_{1}, \ldots, \xi_{m}\right) \in \mathcal{O}_{p}$, we first study the linear equation

$$
\begin{cases}\mathcal{L}(\phi)=-\Delta_{a(\varepsilon y)} \phi+\varepsilon^{2} \phi-W_{\xi^{\prime}} \phi=h & \text { in } \Omega_{p}, \\ \frac{\partial \phi}{\partial \nu}=0 & \text { on } \partial \Omega_{p} .\end{cases}
$$

For the solution of (3.13) satisfying the orthogonality conditions with respect to $Z_{i j}, i=1, \ldots, m, j=0,1, J_{i}$, we prove the following a priori estimate.

Lemma 3.5. There exist $R_{0}>0$ and $p_{m}>1$ such that for any $p>p_{m}$ and any solution $\phi$ of (3.13) with the orthogonality conditions

$$
\int_{\Omega_{p}} \chi_{i} Z_{i j} \phi=0 \quad \forall i=1, \ldots, m, j=0,1, J_{i}
$$

we have

where $C>0$ is independent of $p$.

$$
\|\phi\|_{L^{\infty}\left(\Omega_{p}\right)} \leq C\|h\|_{*},
$$


Proof. Take $R_{0}=2 R_{1}$, with $R_{1}$ as the constant of Lemma 3.4. Since $\xi=\left(\xi_{1}, \ldots, \xi_{m}\right) \in \mathcal{O}_{p}$ and $\varepsilon \mu_{i}=o\left(1 / p^{\kappa}\right)$ for $p$ large enough, we find $B_{R_{1} \mu_{i}}\left(\xi_{i}^{\prime}\right)$ disjointed. Let $h$ be bounded and $\phi$ be a bounded solution to (3.13) satisfying (3.14). We first consider the following inner norm of $\phi$ :

$$
\|\phi\|_{i}=\sup _{y \in \bar{\Omega}_{p} \cap\left(\bigcup_{i=1}^{m} B_{R_{1} \mu_{i}}\left(\xi_{i}^{\prime}\right)\right)}|\phi(y)|,
$$

and claim that there is a constant $C>0$ independent of $p$ such that

$$
\|\phi\|_{L^{\infty}\left(\Omega_{p}\right)} \leq C\left(\|\phi\|_{i}+\|h\|_{*}\right) .
$$

Indeed, set

$$
\widetilde{\phi}(y)=C_{1}\left(\|\phi\|_{i}+\|h\|_{*}\right) \psi(y) \quad \forall y \in \bar{\Omega}_{p} \backslash \bigcup_{i=1}^{m} B_{R_{1} \mu_{i}}\left(\xi_{i}^{\prime}\right),
$$

where $\psi$ is the positive, uniformly bounded barrier constructed by the previous lemma and the constant $C_{1}>0$ is chosen larger if necessary, independent of $p$. Then for $y \in \Omega_{p} \backslash \bigcup_{i=1}^{m} B_{R_{1} \mu_{i}}\left(\xi_{i}^{\prime}\right)$,

$$
\mathcal{L}(\widetilde{\phi} \pm \phi)(y) \geq C_{1}\|h\|_{*}\left\{\sum_{i=1}^{m} \frac{\mu_{i}^{\sigma}}{\left|y-\xi_{i}^{\prime}\right|^{2+\sigma}}+\varepsilon^{2}\right\} \pm h(y) \geq|h(y)| \pm h(y) \geq 0,
$$

for $y \in \partial \Omega_{p} \backslash \bigcup_{i=1}^{m} B_{R_{1} \mu_{i}}\left(\xi_{i}^{\prime}\right)$,

$$
\frac{\partial}{\partial \nu}(\widetilde{\phi} \pm \phi)(y) \geq 0
$$

and for $y \in \Omega_{p} \cap\left(\bigcup_{i=1}^{m} \partial B_{R_{1} \mu_{i}}\left(\xi_{i}^{\prime}\right)\right)$,

$$
(\widetilde{\phi} \pm \phi)(y)>\|\phi\|_{i} \pm \phi(y) \geq|\phi(y)| \pm \phi(y) \geq 0 .
$$

From the maximum principle (see [48]), it follows that $-\widetilde{\phi} \leq \phi \leq \widetilde{\phi}$ on $\bar{\Omega}_{p} \backslash \bigcup_{i=1}^{m} B_{R_{1} \mu_{i}}\left(\xi_{i}^{\prime}\right)$, which implies estimate (3.15).

We prove the lemma by contradiction. Assume that there are sequences of parameters $p_{n} \rightarrow+\infty$, points $\xi^{n}=$ $\left(\xi_{1}^{n}, \ldots, \xi_{m}^{n}\right) \in \mathcal{O}_{p_{n}}$, functions $h_{n}$, and associated solutions $\phi_{n}$ of equation (3.13) with orthogonality conditions (3.14) such that

$$
\left\|\phi_{n}\right\|_{L^{\infty}\left(\Omega_{p_{n}}\right)}=1 \quad \text { and } \quad\left\|h_{n}\right\|_{*} \rightarrow 0, \quad \text { as } n \rightarrow+\infty .
$$

For each $k \in\{1, \ldots, l\}$, we have $\xi_{k}^{n} \in \Omega$ and we consider $\widehat{\phi}_{k}^{n}(z)=\phi_{n}\left(\mu_{k}^{n} z+\left(\xi_{k}^{n}\right)^{\prime}\right)$, where $\mu^{n}=\left(\mu_{1}^{n}, \ldots, \mu_{m}^{n}\right)$, $\left(\xi_{k}^{n}\right)^{\prime}=\xi_{k}^{n} / \varepsilon_{n}$ and $\varepsilon_{n}=\exp \left\{-p_{n} / 4\right\}$. Note that

$$
h_{n}(y)=\left.\left(-\Delta_{a\left(\varepsilon_{n} y\right)} \phi_{n}+\varepsilon_{n}^{2} \phi-W_{\left(\xi^{n}\right)^{\prime}} \phi_{n}\right)\right|_{y=\mu_{k}^{n} z+\left(\xi_{k}^{n}\right)^{\prime}}=\left(\mu_{k}^{n}\right)^{-2}\left[-\Delta_{\widehat{a}_{n}} \widehat{\phi}_{k}^{n}+\varepsilon_{n}^{2}\left(\mu_{k}^{n}\right)^{2} \widehat{\phi}_{k}^{n}-\left(\mu_{k}^{n}\right)^{2} \widehat{W}^{n} \widehat{\phi}_{k}^{n}\right](z),
$$

where

$$
\widehat{a}_{n}(z)=a\left(\varepsilon_{n} \mu_{k}^{n} z+\xi_{k}^{n}\right), \quad \widehat{W}^{n}(z)=W_{\left(\xi^{n}\right)^{\prime}}\left(\mu_{k}^{n} z+\left(\xi_{k}^{n}\right)^{\prime}\right) .
$$

By the expansion of $W^{n}$ in (3.2) and elliptic regularity, $\widehat{\phi}_{k}^{n}$ converges uniformly over compact sets to a bounded solution $\widehat{\phi}_{k}^{\infty}$ of equation (3.4), which satisfies

$$
\int_{\mathbb{R}^{2}} \chi Z_{j} \widehat{\phi}_{k}^{\infty}=0 \quad \text { for } j=0,1,2
$$

Thus $\widehat{\phi}_{k}^{\infty}$ is a linear combination of $Z_{j}, j=0,1,2$. Notice that $\int_{\mathbb{R}^{2}} \chi Z_{j} Z_{t}=0$ for $j \neq t$ and $\int_{\mathbb{R}^{2}} \chi Z_{j}^{2}>0$. Hence (3.17) implies $\widehat{\phi}_{k}^{\infty} \equiv 0$.

As for each $k \in\{l+1, \ldots, m\}$, we have $\xi_{k}^{n} \in \partial \Omega$ and we consider $\widehat{\phi}_{k}^{n}(z)=\phi_{n}\left(\left(A_{k}^{n}\right)^{-1} \mu_{k}^{n} z+\left(\xi_{k}^{n}\right)^{\prime}\right)$, where $A_{k}^{n}: \mathbb{R}^{2} \rightarrow \mathbb{R}^{2}$ is a rotation map such that $A_{k}^{n} \nu_{\Omega_{p_{n}}}\left(\left(\xi_{k}^{n}\right)^{\prime}\right)=\nu_{\mathbb{R}_{+}^{2}}(0)$. Similarly to the above argument, we can get that $\widehat{\phi}_{k}^{n}$ converges uniformly over compact sets to a bounded solution $\widehat{\phi}_{k}^{\infty}$ of equation (3.5), which satisfies

$$
\int_{\mathbb{R}_{+}^{2}} \chi Z_{j} \widehat{\phi}_{k}^{\infty}=0 \quad \text { for } j=0,1 .
$$


Thus $\widehat{\phi}_{k}^{\infty}$ is a linear combination of $Z_{j}, j=0,1$. Notice that $\int_{\mathbb{R}_{+}^{2}} \chi Z_{j} Z_{t}=0$ for $j \neq t$ and $\int_{\mathbb{R}_{+}^{2}} \chi Z_{j}^{2}>0$. Hence (3.18) implies $\widehat{\phi}_{k}^{\infty}=0$. Furthermore, we find that $\lim _{n \rightarrow+\infty}\left\|\phi_{n}\right\|_{i}=0$. But (3.15) and (3.16) tell us $\liminf \inf _{n \rightarrow+\infty}\left\|\phi_{n}\right\|_{i}>0$, which is a contradiction.

Step 3: Proving an a priori estimate for solutions to (3.13) that satisfy orthogonality conditions with respect to $Z_{i j}, j=1, J_{i}$ only.

Lemma 3.6. For p large enough, if $\phi$ solves (3.13) and satisfies

$$
\int_{\Omega_{p}} \chi_{i} Z_{i j} \phi=0 \quad \forall i=1, \ldots, m, j=1, J_{i}
$$

then

$$
\|\phi\|_{L^{\infty}\left(\Omega_{p}\right)} \leq C p\|h\|_{*},
$$

where $C>0$ is independent of $p$.

Proof. According to the results in Lemma 3.4 of [18] and Lemma 3.2 of [43], for simplicity we only consider the validity of estimate (3.20) when the $m$ concentration points $\xi=\left(\xi_{1}, \ldots, \xi_{m}\right) \in \mathcal{O}_{p}$ satisfy the relation $\left|\xi_{i}-\xi_{k}\right| \leq 2 d$ for any $i, k=1, \ldots, m, i \neq k$ and for any $d>0$ sufficiently small, fixed and independent of $p$. Let $R>R_{0}+1$ be a large but fixed number. Denote for $i=1, \ldots, m$,

$$
\widehat{Z}_{i 0}(y)=Z_{i 0}(y)-\frac{1}{\mu_{i}}+a_{i 0} G\left(\varepsilon y, \xi_{i}\right),
$$

where

$$
a_{i 0}=\frac{1}{\mu_{i}\left[H\left(\xi_{i}, \xi_{i}\right)-\frac{4}{c_{i}} \log \left(\varepsilon \mu_{i} R\right)\right]} .
$$

Note that by estimate (2.23), expansions (2.2) and (2.4), and definitions (2.9), (3.3), (3.7) and (3.10),

$$
\frac{1}{8} p \leq-\log \left(\varepsilon \mu_{i} R\right) \leq \frac{1}{2} p,
$$

and

$$
\widehat{Z}_{i 0}(y)=O\left(\frac{G\left(\varepsilon y, \xi_{i}\right)}{p \mu_{i}}\right) .
$$

Let $\eta_{1}$ and $\eta_{2}$ be radial smooth cut-off functions in $\mathbb{R}^{2}$ such that

$$
\begin{array}{llll}
0 \leq \eta_{1} \leq 1 ; & \left|\nabla \eta_{1}\right| \leq C \text { in } \mathbb{R}^{2} ; & \eta_{1} \equiv 1 \text { in } B_{R}(0) ; & \eta_{1} \equiv 0 \text { in } \mathbb{R}^{2} \backslash B_{R+1}(0) ; \\
0 \leq \eta_{2} \leq 1 ; & \left|\nabla \eta_{2}\right| \leq C \text { in } \mathbb{R}^{2} ; & \eta_{2} \equiv 1 \text { in } B_{3 d}(0) ; & \eta_{2} \equiv 0 \text { in } \mathbb{R}^{2} \backslash B_{6 d}(0) .
\end{array}
$$

We set, for $i \in\{1, \ldots, l\}$,

$$
\eta_{i 1}(y)=\eta_{1}\left(\frac{1}{\mu_{i}}\left|y-\xi_{i}^{\prime}\right|\right), \quad \eta_{i 2}(y)=\eta_{2}\left(\varepsilon\left|y-\xi_{i}^{\prime}\right|\right)
$$

and for $i \in\{l+1, \ldots, m\}$,

$$
\eta_{i 1}(y)=\eta_{1}\left(\frac{1}{\mu_{i}}\left|F_{i}^{p}(y)\right|\right), \quad \eta_{i 2}(y)=\eta_{2}\left(\varepsilon\left|F_{i}^{p}(y)\right|\right) .
$$

Now we define the test function

$$
\widetilde{Z}_{i 0}(y)=\eta_{i 1} Z_{i 0}+\left(1-\eta_{i 1}\right) \eta_{i 2} \widehat{Z}_{i 0} .
$$

Given $\phi$ satisfying (3.13) and (3.19), let

$$
\widetilde{\phi}=\phi+\sum_{i=1}^{m} d_{i} \widetilde{Z}_{i 0}+\sum_{i=1}^{m} \sum_{j=1}^{J_{i}} e_{i j} \chi_{i} Z_{i j} .
$$


We will first prove the existence of $d_{i}$ and $e_{i j}$ such that $\widetilde{\phi}$ satisfies the orthogonality condition

$$
\int_{\Omega_{p}} \chi_{i} Z_{i j} \widetilde{\phi}=0 \quad \forall i=1, \ldots, m, j=0,1, J_{i}
$$

Multiplying (3.28) by $\chi_{i} Z_{i j}, i=1, \ldots, m, j=0,1, J_{i}$ and using orthogonality conditions (3.19) and (3.29) together with the fact that $\chi_{i} \chi_{k} \equiv 0$ if $i \neq k$, we get

$$
\begin{gathered}
d_{i} \int_{\Omega_{p}} \chi_{i} Z_{i 0} \widetilde{Z}_{i 0}+\sum_{k \neq i}^{m} d_{k} \int_{\Omega_{p}} \chi_{i} Z_{i 0} \widetilde{Z}_{k 0}+\sum_{t=1}^{J_{i}} e_{i t} \int_{\Omega_{p}} \chi_{i}^{2} Z_{i 0} Z_{i t}=-\int_{\Omega_{p}} \chi_{i} Z_{i 0} \phi, \\
d_{i} \int_{\Omega_{p}} \chi_{i} Z_{i j} \widetilde{Z}_{i 0}+\sum_{k \neq i}^{m} d_{k} \int_{\Omega_{p}} \chi_{i} Z_{i j} \widetilde{Z}_{k 0}+\sum_{t=1}^{J_{i}} e_{i t} \int_{\Omega_{p}} \chi_{i}^{2} Z_{i j} Z_{i t}=0, \quad j=1, J_{i} .
\end{gathered}
$$

Remark that for any $i=1, \ldots, l, \widetilde{Z}_{i 0}$ coincides with $Z_{i 0}$ in $B_{R \mu_{i}}\left(\xi_{i}^{\prime}\right)$, while for any $i=l+1, \ldots, m, \widetilde{Z}_{i 0}$ coincides with $Z_{i 0}$ in the region $\left\{y \in \Omega_{p}:\left|F_{i}^{p}(y)\right| \leq R \mu_{i}\right\}$. Moreover, from definitions (3.8)-(3.9) we can write $z=\frac{1}{\mu_{i}} F_{i}^{p}(y)$ and its inverse $y=\left(\frac{1}{\mu_{i}} F_{i}^{p}\right)^{-1}(z)=\xi_{i}^{\prime}+\frac{1}{\varepsilon} A_{i}^{-1} F_{i}^{-1}\left(\varepsilon \mu_{i} z\right)$ such that $\operatorname{det}\left(\nabla\left(\frac{1}{\mu_{i}} F_{i}^{p}\right)^{-1}(z)\right)=\mu_{i}^{2}+O\left(\varepsilon \mu_{i}^{3}|z|\right)$ holds in the upper half-ball $B_{R \mu_{i}}^{+}(0):=B_{R \mu_{i}}(0) \cap \mathbb{R}_{+}^{2}$. Then for any $i=1, \ldots, l, j=1,2$ and $t=1,2$,

$$
\begin{array}{lrl}
\int_{\Omega_{p}} \chi_{i} Z_{i 0} \widetilde{Z}_{i 0}=\int_{\mathbb{R}^{2}} \chi Z_{0}^{2}=C_{0}>0, & \int_{\Omega_{p}} \chi_{i}^{2} Z_{i 0} Z_{i t}=\int_{\mathbb{R}^{2}} \chi^{2} Z_{0} Z_{t}=0, \\
\int_{\Omega_{p}} \chi_{i} Z_{i j} \widetilde{Z}_{i 0}=\int_{\mathbb{R}^{2}} \chi Z_{j} Z_{0}=0, & \int_{\Omega_{p}} \chi_{i}^{2} Z_{i j} Z_{i t}=\int_{\mathbb{R}^{2}} \chi^{2} Z_{j} Z_{t}=C_{j} \delta_{j t},
\end{array}
$$

where $\delta_{j t}$ denotes the Kronecker's symbol, but for any $i=l+1, \ldots, m$ and $j=t=J_{i}=1$,

$$
\begin{gathered}
\int_{\Omega_{p}} \chi_{i} Z_{i 0} \widetilde{Z}_{i 0}=\int_{\mathbb{R}_{+}^{2}} \chi Z_{0}^{2}\left[1+O\left(\varepsilon \mu_{i}|z|\right)\right]=\frac{C_{0}}{2}+O\left(\varepsilon \mu_{i}\right), \quad \int_{\Omega_{p}} \chi_{i}^{2} Z_{i 0} Z_{i 1}=\int_{\mathbb{R}_{+}^{2}} \chi^{2} Z_{0} Z_{1}\left[1+O\left(\varepsilon \mu_{i}|z|\right)\right]=O\left(\varepsilon \mu_{i}\right), \\
\int_{\Omega_{p}} \chi_{i} Z_{i 1} \widetilde{Z}_{i 0}=\int_{\mathbb{R}_{+}^{2}} \chi Z_{1} Z_{0}\left[1+O\left(\varepsilon \mu_{i}|z|\right)\right]=O\left(\varepsilon \mu_{i}\right), \quad \int_{\Omega_{p}} \chi_{i}^{2} Z_{i 1}^{2}=\int_{\mathbb{R}_{+}^{2}} \chi^{2} Z_{1}^{2}\left[1+O\left(\varepsilon \mu_{i}|z|\right)\right]=\frac{C_{1}}{2}+O\left(\varepsilon \mu_{i}\right) .
\end{gathered}
$$

Moreover, from (3.24) and (3.27) it follows that for any $i=1, \ldots, m$ and $j=0,1, J_{i}$,

$$
\int_{\Omega_{p}} \chi_{i} Z_{i j} \widetilde{Z}_{k 0}=O\left(\frac{\mu_{i} \log p}{p \mu_{k}}\right), \quad \forall k \neq i .
$$

Thus by (3.31),

$$
e_{i j}=\left(-d_{i} \int_{\Omega_{p}} \chi_{i} Z_{i j} \widetilde{Z}_{i 0}-\sum_{k \neq i}^{m} d_{k} \int_{\Omega_{p}} \chi_{i} Z_{i j} \widetilde{Z}_{k 0}\right) / \int_{\Omega_{p}} \chi_{i}^{2} Z_{i j}^{2}, \quad i=1, \ldots, m, j=1, J_{i} .
$$

Furthermore,

$$
\left|e_{i j}\right| \leq \begin{cases}C \sum_{k \neq i}^{m}\left|d_{k}\right| \frac{\mu_{i} \log p}{p \mu_{k}}, & \forall i=1, \ldots, l, \quad j=1,2, \\ C \varepsilon \mu_{i}\left|d_{i}\right|+C \sum_{k \neq i}^{m}\left|d_{k}\right| \frac{\mu_{i} \log p}{p \mu_{k}}, & \forall i=l+1, \ldots, m, j=1 .\end{cases}
$$

We need just to show that $d_{i}$ is well defined. From (3.30) we can easily get that for any $i=1, \ldots, l$,

$$
d_{i} C_{0}+\sum_{k \neq i}^{m} d_{k} O\left(\frac{\mu_{i} \log p}{p \mu_{k}}\right)=-\int_{\Omega_{p}} \chi_{i} Z_{i 0} \phi
$$


and for any $i=l+1, \ldots, m$,

$$
\frac{1}{2} d_{i} C_{0}\left[1+O\left(\varepsilon \mu_{i}\right)\right]+\sum_{k \neq i}^{m} d_{k} O\left(\frac{\mu_{i} \log p}{p \mu_{k}}\right)+e_{i 1} O\left(\varepsilon \mu_{i}\right)=-\int_{\Omega_{p}} \chi_{i} Z_{i 0} \phi,
$$

where $e_{i 1}$ is defined in (3.32) and satisfies

$$
e_{i 1}=d_{i} O\left(\varepsilon \mu_{i}\right)+\sum_{k \neq i}^{m} d_{k} O\left(\frac{\mu_{i} \log p}{p \mu_{k}}\right) .
$$

We denote $\mathcal{A}$ the coefficient matrix of equations (3.34)-(3.35). By the above estimates, it is clear that $\mathcal{M}^{-1} \mathcal{A M}$ is diagonally dominant and thus invertible, where $\mathcal{M}=\operatorname{diag}\left(\mu_{1}, \ldots, \mu_{m}\right)$. Hence $\mathcal{A}$ is also invertible and $\left(d_{1}, \ldots, d_{m}\right)$ is well defined.

Estimate (3.20) is a direct consequence of the following two claims.

Claim 1. Let $\mathcal{L}=-\Delta_{a(\varepsilon y)}+\varepsilon^{2}-W_{\xi^{\prime}}$, then for any $i=1, \ldots, m$ and $j=1, J_{i}$,

$$
\left\|\mathcal{L}\left(\chi_{i} Z_{i j}\right)\right\|_{*} \leq \frac{C}{\mu_{i}}, \quad \quad\left\|\mathcal{L}\left(\widetilde{Z}_{i 0}\right)\right\|_{*} \leq C \frac{\log p}{p \mu_{i}} .
$$

Claim 2. For any $i=1, \ldots, m$ and $j=1, J_{i}$,

$$
\left|d_{i}\right| \leq C p \mu_{i}\|h\|_{*}, \quad \quad\left|e_{i j}\right| \leq C \mu_{i} \log p\|h\|_{*} .
$$

In fact, the definition of $\widetilde{\phi}$ in (3.28) tells us

$$
\begin{cases}\mathcal{L}(\widetilde{\phi})=h+\sum_{i=1}^{m} d_{i} \mathcal{L}\left(\widetilde{Z}_{i 0}\right)+\sum_{i=1}^{m} \sum_{j=1}^{J_{i}} e_{i j} \mathcal{L}\left(\chi_{i} Z_{i j}\right) & \text { in } \quad \Omega_{p} \\ \frac{\partial \widetilde{\phi}}{\partial \nu}=0 & \text { on } \quad \partial \Omega_{p} .\end{cases}
$$

Then by Lemma 3.5, we obtain

$$
\|\widetilde{\phi}\|_{L^{\infty}\left(\Omega_{p}\right)} \leq C\left\{\|h\|_{*}+\sum_{i=1}^{m}\left|d_{i}\right|\left\|\mathcal{L}\left(\widetilde{Z}_{i 0}\right)\right\|_{*}+\sum_{i=1}^{m} \sum_{j=1}^{J_{i}}\left|e_{i j}\right|\left\|\mathcal{L}\left(\chi_{i} Z_{i j}\right)\right\|_{*}\right\} \leq C \log p\|h\|_{*} .
$$

Using the definition of $\widetilde{\phi}$ again and the fact that

$$
\left\|\widetilde{Z}_{i 0}\right\|_{L^{\infty}\left(\Omega_{p}\right)} \leq \frac{C}{\mu_{i}} \quad \text { and } \quad\left\|\chi_{i} Z_{i j}\right\|_{L^{\infty}\left(\Omega_{p}\right)} \leq \frac{C}{\mu_{i}}, \quad \forall i=1, \ldots, m, j=1, J_{i},
$$

estimate (3.20) then follows from estimate (3.38) and Claim 2.

Proof of Claim 1. Observe that

$$
\mathcal{L}\left(\chi_{i} Z_{i j}\right)=\chi_{i} \mathcal{L}\left(Z_{i j}\right)-2 \nabla \chi_{i} \nabla Z_{i j}-Z_{i j}\left[\Delta \chi_{i}+\varepsilon \nabla \log a(\varepsilon y) \nabla \chi_{i}\right] .
$$

For any $i=1, \ldots, l$ and $j=1,2$, we write $z_{i}:=y-\xi_{i}^{\prime}$ and note that in the region $\left|z_{i}\right|=\left|y-\xi_{i}^{\prime}\right| \leq \mu_{i}\left(R_{0}+1\right)$, by (3.2), (3.3), (3.4) and (3.7),

$$
\begin{aligned}
\mathcal{L}\left(Z_{i j}\right) & =\left(-\Delta-W_{\xi^{\prime}}\right)\left[\frac{1}{\mu_{i}} Z_{j}\left(\frac{y-\xi_{i}^{\prime}}{\mu_{i}}\right)\right]-\varepsilon \nabla \log a(\varepsilon y) \nabla\left[\frac{1}{\mu_{i}} Z_{j}\left(\frac{y-\xi_{i}^{\prime}}{\mu_{i}}\right)\right]+\frac{\varepsilon^{2}}{\mu_{i}} Z_{j}\left(\frac{y-\xi_{i}^{\prime}}{\mu_{i}}\right) \\
& =O\left(\frac{1}{p \mu_{i}} \cdot \frac{8 \mu_{i}^{2}}{\left(\mu_{i}^{2}+\left|y-\xi_{i}^{\prime}\right|^{2}\right)^{2}}\right)+O\left(\frac{\varepsilon}{\mu_{i}^{2}+\left|y-\xi_{i}^{\prime}\right|^{2}}\right)+O\left(\frac{\varepsilon^{2}}{\left(\mu_{i}^{2}+\left|y-\xi_{i}^{\prime}\right|^{2}\right)^{1 / 2}}\right),
\end{aligned}
$$


and then, by (3.40),

$$
\mathcal{L}\left(\chi_{i} Z_{i j}\right)=O\left(\frac{\mu_{i} p^{-1}}{\left(\mu_{i}^{2}+\left|y-\xi_{i}^{\prime}\right|^{2}\right)^{2}}\right)+O\left(\frac{\mu_{i}^{-1}}{\mu_{i}^{2}+\left|y-\xi_{i}^{\prime}\right|^{2}}\right)+O\left(\frac{\mu_{i}^{-2}}{\left(\mu_{i}^{2}+\left|y-\xi_{i}^{\prime}\right|^{2}\right)^{1 / 2}}\right) .
$$

As for any $i=l+1, \ldots, m$, owing to $F_{i}^{p}\left(\xi_{i}^{\prime}\right)=(0,0)$ and $\nabla F_{i}^{p}\left(\xi_{i}^{\prime}\right)=A_{i}$, we know

$$
\nabla_{y}=A_{i} \nabla_{z_{i}}+O\left(\varepsilon\left|z_{i}\right|\right) \nabla_{z_{i}} \quad \text { and } \quad-\Delta_{y}=-\Delta_{z_{i}}+O\left(\varepsilon\left|z_{i}\right|\right) \nabla_{z_{i}}^{2}+O(\varepsilon) \nabla_{z_{i}}
$$

where

$$
z_{i}:=F_{i}^{p}(y)=\frac{1}{\varepsilon} F_{i}\left(A_{i}\left(\varepsilon y-\xi_{i}\right)\right)=A_{i}\left(y-\xi_{i}^{\prime}\right)\left\{1+O\left(\varepsilon A_{i}\left(y-\xi_{i}^{\prime}\right)\right)\right\} .
$$

In the region $\left|z_{i}\right|=\left|F_{i}^{p}(y)\right| \leq \mu_{i}\left(R_{0}+1\right)$, by (3.2), (3.3), (3.5), (3.10), (3.42) and (3.43),

$$
\begin{aligned}
\mathcal{L}\left(Z_{i 1}\right) & =\left(-\Delta_{z_{i}}-W_{\xi^{\prime}}\right)\left[\frac{1}{\mu_{i}} Z_{1}\left(\frac{z_{i}}{\mu_{i}}\right)\right]+\left(\varepsilon^{2}+O\left(\varepsilon\left|z_{i}\right|\right) \nabla_{z_{i}}^{2}+O(\varepsilon) \nabla_{z_{i}}\right)\left[\frac{1}{\mu_{i}} Z_{1}\left(\frac{z_{i}}{\mu_{i}}\right)\right] \\
& =O\left(\frac{1}{p \mu_{i}} \cdot \frac{8 \mu_{i}^{2}}{\left(\mu_{i}^{2}+\left|z_{i}\right|^{2}\right)^{2}}\right)+O\left(\frac{\varepsilon}{\mu_{i}^{2}+\left|z_{i}\right|^{2}}\right)+O\left(\frac{\varepsilon^{2}}{\left(\mu_{i}^{2}+\left|z_{i}\right|^{2}\right)^{1 / 2}}\right) .
\end{aligned}
$$

Thus by (3.40),

$$
\mathcal{L}\left(\chi_{i} Z_{i 1}\right)=O\left(\frac{\mu_{i} p^{-1}}{\left(\mu_{i}^{2}+\left|F_{i}^{p}(y)\right|^{2}\right)^{2}}\right)+O\left(\frac{\mu_{i}^{-1}}{\mu_{i}^{2}+\left|F_{i}^{p}(y)\right|^{2}}\right)+O\left(\frac{\mu_{i}^{-2}}{\left(\mu_{i}^{2}+\left|F_{i}^{p}(y)\right|^{2}\right)^{1 / 2}}\right) .
$$

Hence by (3.41), (3.43), (3.44) and the definition of $\|\cdot\|_{*}$ in $(2.30)$, we obtain $\left\|\mathcal{L}\left(\chi_{i} Z_{i j}\right)\right\|_{*}=O\left(1 / \mu_{i}\right)$ for all $i=1, \ldots, m$ and $j=1, J_{i}$.

We now prove the second inequality in (3.36). In fact,

$$
\begin{aligned}
\mathcal{L}\left(\widetilde{Z}_{i 0}\right)= & \eta_{i 1} \mathcal{L}\left(Z_{i 0}-\widehat{Z}_{i 0}\right)+\eta_{i 2} \mathcal{L}\left(\widehat{Z}_{i 0}\right)-\left(Z_{i 0}-\widehat{Z}_{i 0}\right) \Delta_{a(\varepsilon y)} \eta_{i 1}-2 \nabla \eta_{i 1} \nabla\left(Z_{i 0}-\widehat{Z}_{i 0}\right) \\
& -2 \nabla \eta_{i 2} \nabla \widehat{Z}_{i 0}-\widehat{Z}_{i 0} \Delta_{a(\varepsilon y)} \eta_{i 2} .
\end{aligned}
$$

Recalling that $z_{i}=y-\xi_{i}^{\prime}$ for any $i=1, \ldots, l$, but $z_{i}=F_{i}^{p}(y)$ for any $i=l+1, \ldots, m$, we now consider the four regions

$$
\begin{array}{clrl}
\Omega_{1}=\left\{y \in \Omega_{p}|| z_{i} \mid \leq \mu_{i} R\right\}, & & \Omega_{2}=\left\{y \in \Omega_{p}\left|\mu_{i} R<\right| z_{i} \mid \leq \mu_{i}(R+1)\right\}, \\
\Omega_{3}=\left\{y \in \Omega_{p}\left|\mu_{i}(R+1)<\right| z_{i} \mid \leq 3 d / \varepsilon\right\}, & & \Omega_{4}=\left\{y \in \Omega_{p}|3 d / \varepsilon<| z_{i} \mid \leq 6 d / \varepsilon\right\} .
\end{array}
$$

Notice first that, by (3.3), (3.7), (3.10) and (3.43),

$$
\left|Z_{i 0}-\frac{1}{\mu_{i}}\right|=\frac{2 \mu_{i}}{\mu_{i}^{2}+\left|z_{i}\right|^{2}}=O\left(\frac{\mu_{i}}{\left(\mu_{i}+\left|y-\xi_{i}^{\prime}\right|\right)^{2}}\right),
$$

and for $\mu_{i} R<\left|z_{i}\right| \leq 6 d / \varepsilon$, by (3.21)-(3.22),

$$
Z_{i 0}-\widehat{Z}_{i 0}=\frac{1}{\mu_{i}}-a_{i 0} G\left(\varepsilon y, \xi_{i}\right)=\frac{1}{\mu_{i}\left[H\left(\xi_{i}, \xi_{i}\right)-\frac{4}{c_{i}} \log \left(\varepsilon \mu_{i} R\right)\right]}\left[\frac{4}{c_{i}} \log \frac{\left|y-\xi_{i}^{\prime}\right|}{\mu_{i} R}+O\left(\varepsilon^{\alpha}\left|y-\xi_{i}^{\prime}\right|^{\alpha}\right)\right] .
$$

In $\Omega_{1}$, by (3.45),

$$
\mathcal{L}\left(\widetilde{Z}_{i 0}\right)=\mathcal{L}\left(Z_{i 0}\right)=\left[-\Delta_{a(\varepsilon y)} Z_{i 0}-\frac{8 \mu_{i}^{2}}{\left(\mu_{i}^{2}+\left|y-\xi_{i}^{\prime}\right|^{2}\right)^{2}} Z_{i 0}\right]+\left[\frac{8 \mu_{i}^{2}}{\left(\mu_{i}^{2}+\left|y-\xi_{i}^{\prime}\right|^{2}\right)^{2}}-W_{\xi^{\prime}}\right] Z_{i 0}+\varepsilon^{2} Z_{i 0} .
$$

Note that, by (3.2),

$$
\left[\frac{8 \mu_{i}^{2}}{\left(\mu_{i}^{2}+\left|y-\xi_{i}^{\prime}\right|^{2}\right)^{2}}-W_{\xi^{\prime}}\right] Z_{i 0}=O\left(\frac{\mu_{i} p^{-1}}{\left(\mu_{i}^{2}+\left|y-\xi_{i}^{\prime}\right|^{2}\right)^{2}}\right), \quad \forall y \in \Omega_{1} \cup \Omega_{2} .
$$

Hence in $\Omega_{1}$, by (3.11),

$$
\mathcal{L}\left(\widetilde{Z}_{i 0}\right)=\mathcal{L}\left(Z_{i 0}\right)=O\left(\frac{1}{p \mu_{i}^{3}}\right)
$$


In $\Omega_{2}$, by (3.21) and (3.45),

$$
\begin{aligned}
\mathcal{L}\left(\widetilde{Z}_{i 0}\right)= & {\left[-\Delta_{a(\varepsilon y)} Z_{i 0}-\frac{8 \mu_{i}^{2}}{\left(\mu_{i}^{2}+\left|y-\xi_{i}^{\prime}\right|^{2}\right)^{2}} Z_{i 0}\right]+\left[\frac{8 \mu_{i}^{2}}{\left(\mu_{i}^{2}+\left|y-\xi_{i}^{\prime}\right|^{2}\right)^{2}}-W_{\xi^{\prime}}\right] Z_{i 0}+\varepsilon^{2}\left(Z_{i 0}-\frac{1}{\mu_{i}}\right) } \\
& +W_{\xi^{\prime}}\left(1-\eta_{i 1}\right)\left(Z_{i 0}-\widehat{Z}_{i 0}\right)+\frac{\varepsilon^{2}}{\mu_{i}} \eta_{i 1}-2 \nabla \eta_{i 1} \nabla\left(Z_{i 0}-\widehat{Z}_{i 0}\right)-\left(Z_{i 0}-\widehat{Z}_{i 0}\right) \Delta_{a(\varepsilon y)} \eta_{i 1} .
\end{aligned}
$$

Note that in $\Omega_{2}$, by (3.23) and (3.47),

$$
\left|Z_{i 0}-\widehat{Z}_{i 0}\right|=O\left(\frac{1}{p \mu_{i} R}\right) \quad \text { and } \quad\left|\nabla\left(Z_{i 0}-\widehat{Z}_{i 0}\right)\right|=O\left(\frac{1}{p \mu_{i}^{2} R}\right) .
$$

Moreover $\left|\nabla \eta_{i 1}\right|=O\left(\mu_{i}^{-1}\right),\left|\Delta_{a(\varepsilon y)} \eta_{i 1}\right|=O\left(\mu_{i}^{-2}\right)$. Hence in $\Omega_{2}$, by (3.2), (3.11), (3.46), (3.48) and (3.50),

$$
\mathcal{L}\left(\widetilde{Z}_{i 0}\right)=O\left(\frac{1}{p \mu_{i}^{3} R}\right) .
$$

In $\Omega_{3}$, by (3.11), (3.21), (3.45) and (3.46),

$$
\begin{aligned}
\mathcal{L}\left(\widetilde{Z}_{i 0}\right) & =\mathcal{L}\left(\widehat{Z}_{i 0}\right)=\mathcal{L}\left(Z_{i 0}\right)-\mathcal{L}\left(Z_{i 0}-\widehat{Z}_{i 0}\right) \\
& =\left[\frac{8 \mu_{i}^{2}}{\left(\mu_{i}^{2}+\left|y-\xi_{i}^{\prime}\right|^{2}\right)^{2}}-W_{\xi^{\prime}}\right] Z_{i 0}+W_{\xi^{\prime}}\left[\frac{1}{\mu_{i}}-a_{i 0} G\left(\varepsilon y, \xi_{i}\right)\right]+O\left(\frac{\varepsilon \mu_{i}}{\left(\mu_{i}+\left|y-\xi_{i}^{\prime}\right|\right)^{3}}\right)+O\left(\frac{\varepsilon^{2} \mu_{i}}{\left(\mu_{i}+\left|y-\xi_{i}^{\prime}\right|\right)^{2}}\right) \\
& \equiv A_{1}+A_{2}+O\left(\frac{\varepsilon \mu_{i}}{\left(\mu_{i}+\left|y-\xi_{i}^{\prime}\right|\right)^{3}}\right)+O\left(\frac{\varepsilon^{2} \mu_{i}}{\left(\mu_{i}+\left|y-\xi_{i}^{\prime}\right|\right)^{2}}\right) .
\end{aligned}
$$

To estimate the first two terms, we need to decompose $\Omega_{3}$ into some subregions:

$$
\begin{gathered}
\Omega_{3, i}=\left\{y \in \Omega_{3}\left|\mu_{i}(R+1)<\right| z_{i} \mid \leq \mu_{i}^{1 / 2} /\left(3 \varepsilon^{1 / 2} p^{2 \kappa}\right)\right\} \\
\Omega_{3, k}=\left\{y \in \Omega_{3}|| z_{k} \mid \leq \mu_{k}^{1 / 2} /\left(3 \varepsilon^{1 / 2} p^{2 \kappa}\right)\right\}, \quad k \neq i, \quad \text { and } \quad \widetilde{\Omega}_{3}=\Omega_{3} \backslash \bigcup_{t=1}^{m} \Omega_{3, t} .
\end{gathered}
$$

From (3.1), (3.2) and (3.43) we get

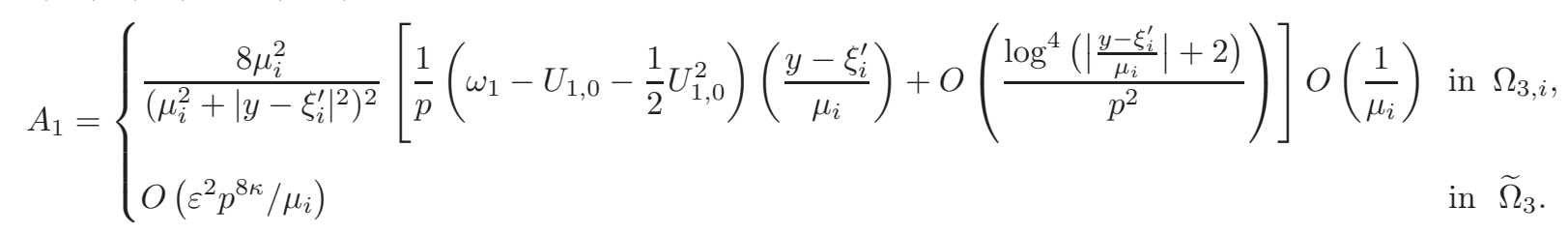

Moreover, by (3.23) and (3.47),

$$
A_{2}=\frac{8 \mu_{i}^{2}}{\left(\mu_{i}^{2}+\left|y-\xi_{i}^{\prime}\right|^{2}\right)^{2}} O\left(\frac{\log \left|y-\xi_{i}^{\prime}\right|-\log \mu_{i} R+\varepsilon^{\alpha}\left|y-\xi_{i}^{\prime}\right|^{\alpha}}{p \mu_{i}}\right) \quad \text { in } \Omega_{3, i} \cup \widetilde{\Omega}_{3} .
$$

Then in $\Omega_{3, i} \cup \widetilde{\Omega}_{3}$, by (2.6) and (2.13),

$$
\mathcal{L}\left(\widetilde{Z}_{i 0}\right)=O\left(\frac{1}{\left(\mu_{i}^{2}+\left|y-\xi_{i}^{\prime}\right|^{2}\right)^{(3+\sigma) / 2}} \cdot \frac{\mu_{i}^{\sigma}}{p}\right)+O\left(\frac{\log \left|y-\xi_{i}^{\prime}\right|-\log \mu_{i} R}{\left(\mu_{i}^{2}+\left|y-\xi_{i}^{\prime}\right|^{2}\right)^{2}} \cdot \frac{\mu_{i}}{p}\right)
$$

with $\sigma>0$ small but fixed, independent of $p$. In $\Omega_{3, k}$ with $k \neq i$, by (3.2), (3.11), (3.24) and (3.46),

$$
\begin{aligned}
\mathcal{L}\left(\widetilde{Z}_{i 0}\right) & =\mathcal{L}\left(\widehat{Z}_{i 0}\right)=\frac{8 \mu_{i}^{2}}{\left(\mu_{i}^{2}+\left|y-\xi_{i}^{\prime}\right|^{2}\right)^{2}} Z_{i 0}-\left[\Delta_{a(\varepsilon y)} Z_{i 0}+\frac{8 \mu_{i}^{2}}{\left(\mu_{i}^{2}+\left|y-\xi_{i}^{\prime}\right|^{2}\right)^{2}} Z_{i 0}\right]+\varepsilon^{2}\left(Z_{i 0}-\frac{1}{\mu_{i}}\right)-W_{\xi^{\prime}} \widehat{Z}_{i 0} \\
& =O\left(\frac{8 \mu_{k}^{2}}{\left(\mu_{k}^{2}+\left|y-\xi_{k}^{\prime}\right|^{2}\right)^{2}} \cdot \frac{\log p}{p \mu_{i}}\right) .
\end{aligned}
$$


In $\Omega_{4}$, by (3.45),

$$
\begin{aligned}
\mathcal{L}\left(\widetilde{Z}_{i 0}\right)= & \frac{8 \mu_{i}^{2}}{\left(\mu_{i}^{2}+\left|y-\xi_{i}^{\prime}\right|^{2}\right)^{2}} \eta_{i 2} Z_{i 0}-\eta_{i 2}\left[\Delta_{a(\varepsilon y)} Z_{i 0}+\frac{8 \mu_{i}^{2}}{\left(\mu_{i}^{2}+\left|y-\xi_{i}^{\prime}\right|^{2}\right)^{2}} Z_{i 0}\right]+\varepsilon^{2} \eta_{i 2}\left(Z_{i 0}-\frac{1}{\mu_{i}}\right) \\
& -\eta_{i 2} W_{\xi^{\prime}} \widehat{Z}_{i 0}-2 \nabla \eta_{i 2} \nabla \widehat{Z}_{i 0}-\widehat{Z}_{i 0} \Delta_{a(\varepsilon y)} \eta_{i 2} .
\end{aligned}
$$

By (2.23) and (3.1) we find $W_{\xi^{\prime}}=O\left(\varepsilon^{4-\sigma}\right)$ in $\Omega_{4}$. In addition, $\left|\nabla \eta_{i 2}\right|=O(\varepsilon / d),\left|\Delta_{a(\varepsilon y)} \eta_{i 2}\right|=O\left(\varepsilon^{2} / d^{2}\right)$,

$$
\left|\widehat{Z}_{i 0}\right|=O\left(\frac{|\log d|}{p \mu_{i}}\right) \quad \text { and } \quad\left|\nabla \widehat{Z}_{i 0}\right|=O\left(\frac{\varepsilon}{p d \mu_{i}}\right) .
$$

Hence by (3.11) and (3.46),

$$
\mathcal{L}\left(\widetilde{Z}_{i 0}\right)=O\left(\frac{\varepsilon^{2}|\log d|}{p d^{2} \mu_{i}}\right) .
$$

Combining (2.30), (3.49), (3.51), (3.52), (3.53) and (3.55), we arrive at

$$
\left\|\mathcal{L}\left(\widetilde{Z}_{i 0}\right)\right\|_{*}=O\left(\frac{\log p}{p \mu_{i}}\right), \quad \forall i=1, \ldots, m .
$$

Proof of Claim 2. Multiplying equation (3.37) by $a(\varepsilon y) \widetilde{Z}_{i 0}$, integrating by parts and using the relations (3.38)(3.39), we get

$$
\begin{aligned}
& \sum_{k=1}^{m} d_{k} \int_{\Omega_{p}} a(\varepsilon y) \widetilde{Z}_{k 0} \mathcal{L}\left(\widetilde{Z}_{i 0}\right) \\
& \quad=-\int_{\Omega_{p}} a(\varepsilon y) h \widetilde{Z}_{i 0}+\int_{\Omega_{p}} a(\varepsilon y) \widetilde{\phi} \mathcal{L}\left(\widetilde{Z}_{i 0}\right)-\sum_{k=1}^{m} \sum_{t=1}^{J_{k}} e_{k t} \int_{\Omega_{p}} a(\varepsilon y) \chi_{k} Z_{k t} \mathcal{L}\left(\widetilde{Z}_{i 0}\right) \\
& \quad \leq C \frac{\|h\|_{*}}{\mu_{i}}+C\left\|\mathcal{L}\left(\widetilde{Z}_{i 0}\right)\right\|_{*}\left(\|\widetilde{\phi}\|_{L^{\infty}\left(\Omega_{p}\right)}+\sum_{k=1}^{m} \sum_{t=1}^{J_{k}} \frac{1}{\mu_{k}}\left|e_{k t}\right|\right) \\
& \quad \leq C \frac{\|h\|_{*}}{\mu_{i}}+C\left\|\mathcal{L}\left(\widetilde{Z}_{i 0}\right)\right\|_{*}\left[\|h\|_{*}+\sum_{k=1}^{m}\left|d_{k}\right|\left\|\mathcal{L}\left(\widetilde{Z}_{k 0}\right)\right\|_{*}+\sum_{k=1}^{m} \sum_{t=1}^{J_{k}}\left|e_{k t}\right|\left(\frac{1}{\mu_{k}}+\left\|\mathcal{L}\left(\chi_{k} Z_{k t}\right)\right\|_{*}\right)\right]
\end{aligned}
$$

where we have applied the following inequality

$$
\int_{\Omega_{p}} \frac{\mu_{i}^{\sigma}}{\left(\left|y-\xi_{i}^{\prime}\right|+\mu_{i}\right)^{2+\sigma}} \leq C, \quad \forall i=1, \ldots, m .
$$

But estimates (3.33) and (3.36) imply that for any $i=1, \ldots, m$,

$$
\left|d_{i}\right|\left|\int_{\Omega_{p}} a(\varepsilon y) \widetilde{Z}_{i 0} \mathcal{L}\left(\widetilde{Z}_{i 0}\right)\right| \leq C \frac{\|h\|_{*}}{\mu_{i}}+C \sum_{k=1}^{m} \frac{\left|d_{k}\right| \log ^{2} p}{p^{2} \mu_{i} \mu_{k}}+\sum_{k \neq i}^{m}\left|d_{k} \int_{\Omega_{p}} a(\varepsilon y) \widetilde{Z}_{k 0} \mathcal{L}\left(\widetilde{Z}_{i 0}\right)\right| .
$$

From (3.21), (3.27) and (3.45) we decompose

$$
\int_{\Omega_{p}} a(\varepsilon y) \widetilde{Z}_{i 0} \mathcal{L}\left(\widetilde{Z}_{i 0}\right)=J+K
$$


where

$$
\begin{aligned}
J= & \int_{\Omega_{p}} a(\varepsilon y) \widetilde{Z}_{i 0}\left[\eta_{i 1} \mathcal{L}\left(Z_{i 0}-\widehat{Z}_{i 0}\right)+\eta_{i 2} \mathcal{L}\left(\widehat{Z}_{i 0}\right)\right] \\
= & \int_{\Omega_{p}} a(\varepsilon y) \eta_{i 2}^{2}\left\{Z_{i 0}-\left(1-\eta_{i 1}\right)\left[\frac{1}{\mu_{i}}-a_{i 0} G\left(\varepsilon y, \xi_{i}\right)\right]\right\} \times\left\{W_{\xi^{\prime}}\left(1-\eta_{i 1}\right)\left[\frac{1}{\mu_{i}}-a_{i 0} G\left(\varepsilon y, \xi_{i}\right)\right]\right. \\
& \left.-\left[\Delta_{a(\varepsilon y)} Z_{i 0}+\frac{8 \mu_{i}^{2}}{\left(\mu_{i}^{2}+\left|y-\xi_{i}^{\prime}\right|^{2}\right)^{2}} Z_{i 0}\right]+\left[\frac{8 \mu_{i}^{2}}{\left(\mu_{i}^{2}+\left|y-\xi_{i}^{\prime}\right|^{2}\right)^{2}}-W_{\xi^{\prime}}\right] Z_{i 0}+\varepsilon^{2}\left(Z_{i 0}-\frac{1}{\mu_{i}}\right)+\frac{\varepsilon^{2}}{\mu_{i}} \eta_{i 1}\right\},
\end{aligned}
$$

and

$$
K=\int_{\Omega_{p}} a(\varepsilon y) \widetilde{Z}_{i 0}\left[-\left(Z_{i 0}-\widehat{Z}_{i 0}\right) \Delta_{a(\varepsilon y)} \eta_{i 1}-2 \nabla \eta_{i 1} \nabla\left(Z_{i 0}-\widehat{Z}_{i 0}\right)-2 \nabla \eta_{i 2} \nabla \widehat{Z}_{i 0}-\widehat{Z}_{i 0} \Delta_{a(\varepsilon y)} \eta_{i 2}\right] .
$$

Let us analyze first the behavior of $J$. By (3.1), (3.2), (3.7), (3.10), (3.23) and (3.47),

$$
\begin{aligned}
& \int_{\left|z_{i}\right| \leq \mu_{i}^{1 / 2} /\left(3 \varepsilon^{1 / 2} p^{2 \kappa}\right)} a(\varepsilon y) \eta_{i 2}^{2}\left\{Z_{i 0}-\left(1-\eta_{i 1}\right)\left[\frac{1}{\mu_{i}}-a_{i 0} G\left(\varepsilon y, \xi_{i}\right)\right]\right\} \times W_{\xi^{\prime}}\left(1-\eta_{i 1}\right)\left[\frac{1}{\mu_{i}}-a_{i 0} G\left(\varepsilon y, \xi_{i}\right)\right] d y \\
= & O\left(\frac{1}{p \mu_{i}^{2} R}\right) .
\end{aligned}
$$

By (3.7), (3.10), (3.11), (3.23) and (3.47),

$$
\begin{aligned}
& \int_{\left|z_{i}\right| \leq \mu_{i}^{1 / 2} /\left(3 \varepsilon^{1 / 2} p^{2 \kappa}\right)} a(\varepsilon y) \eta_{i 2}^{2}\left\{Z_{i 0}-\left(1-\eta_{i 1}\right)\left[\frac{1}{\mu_{i}}-a_{i 0} G\left(\varepsilon y, \xi_{i}\right)\right]\right\} \times\left[\Delta_{a(\varepsilon y)} Z_{i 0}+\frac{8 \mu_{i}^{2}}{\left(\mu_{i}^{2}+\left|y-\xi_{i}^{\prime}\right|^{2}\right)^{2}} Z_{i 0}\right] d y \\
= & O\left(\frac{\varepsilon}{\mu_{i}}\right) .
\end{aligned}
$$

By (3.7), (3.10), (3.23), (3.46) and (3.47),

$$
\begin{aligned}
& \int_{\left|z_{i}\right| \leq \mu_{i}^{1 / 2} /\left(3 \varepsilon^{1 / 2} p^{2 \kappa}\right)} a(\varepsilon y) \eta_{i 2}^{2}\left\{Z_{i 0}-\left(1-\eta_{i 1}\right)\left[\frac{1}{\mu_{i}}-a_{i 0} G\left(\varepsilon y, \xi_{i}\right)\right]\right\} \times\left[\varepsilon^{2}\left(Z_{i 0}-\frac{1}{\mu_{i}}\right)+\frac{\varepsilon^{2}}{\mu_{i}} \eta_{i 1}\right] d y \\
= & O\left(p \varepsilon^{2}\right) .
\end{aligned}
$$

By (3.2), (3.7), (3.10), (3.23) and (3.47),

$$
\begin{aligned}
& \int_{\left|z_{i}\right| \leq \mu_{i}^{1 / 2} /\left(3 \varepsilon^{1 / 2} p^{2 \kappa}\right)} a(\varepsilon y) \eta_{i 2}^{2}\left(1-\eta_{i 1}\right)\left[\frac{1}{\mu_{i}}-a_{i 0} G\left(\varepsilon y, \xi_{i}\right)\right]\left[\frac{8 \mu_{i}^{2}}{\left(\mu_{i}^{2}+\left|y-\xi_{i}^{\prime}\right|^{2}\right)^{2}}-W_{\xi^{\prime}}\right] Z_{i 0} d y \\
= & \int_{\mu_{i} R<\left|z_{i}\right| \leq \mu_{i}^{1 / 2} /\left(3 \varepsilon^{1 / 2} p^{2 \kappa}\right)} \frac{8 \mu_{i}^{2} a(\varepsilon y)}{\left(\mu_{i}^{2}+\left|y-\xi_{i}^{\prime}\right|^{2}\right)^{2}}\left[\frac{1}{p}\left(\omega_{1}-U_{1,0}-\frac{1}{2} U_{1,0}^{2}\right)\left(\frac{y-\xi_{i}^{\prime}}{\mu_{i}}\right)+O\left(\frac{1}{p^{2}} \log ^{4}\left|\frac{y-\xi_{i}^{\prime}}{\mu_{i}}\right|\right)\right] \\
& \times O\left(\frac{\log \left|y-\xi_{i}^{\prime}\right|-\log \left(\mu_{i} R\right)+\varepsilon^{\alpha}\left|y-\xi_{i}^{\prime}\right|^{\alpha}}{p \mu_{i}^{2}}\right) d y \\
= & O\left(\frac{1}{p^{2} \mu_{i}^{2} R}\right) .
\end{aligned}
$$

While by (3.1), (3.2), (3.24), (3.27) and (3.53), 


$$
\begin{aligned}
& \int_{\left|z_{i}\right|>\mu_{i}^{1 / 2} /\left(3 \varepsilon^{1 / 2} p^{2 \kappa}\right)} a(\varepsilon y) \widetilde{Z}_{i 0}\left[\eta_{i 1} \mathcal{L}\left(Z_{i 0}-\widehat{Z}_{i 0}\right)+\eta_{i 2} \mathcal{L}\left(\widehat{Z}_{i 0}\right)\right] d y \\
= & \sum_{k \neq i}^{m} \int_{\Omega_{3, k}} a(\varepsilon y) \eta_{i 2}^{2} \widehat{Z}_{i 0} \mathcal{L}\left(\widehat{Z}_{i 0}\right) d y+\int_{\widetilde{\Omega}_{3} \cup \Omega_{4}} a(\varepsilon y) \eta_{i 2}^{2} \widehat{Z}_{i 0} \mathcal{L}\left(\widehat{Z}_{i 0}\right) d y \\
= & \sum_{k \neq i}^{m} O\left(\int_{0}^{\mu_{k}^{1 / 2} /\left(3 \varepsilon^{1 / 2} p^{2 \kappa}\right)} \frac{8 \mu_{k}^{2}}{\left(\mu_{k}^{2}+r^{2}\right)^{2}} \frac{\log ^{2} p}{p^{2} \mu_{i}^{2}} r d r\right)+O\left(\int_{\mu_{i}^{1 / 2} /\left(3 \varepsilon^{1 / 2} p^{2 \kappa}\right)}^{6 d / \varepsilon} \frac{8 \mu_{i}^{2}}{\left(\mu_{i}^{2}+r^{2}\right)^{2}} \frac{\log \varepsilon r}{p \mu_{i}^{2}} r d r\right) \\
= & O\left(\frac{\log ^{2} p}{p^{2} \mu_{i}^{2}}\right) .
\end{aligned}
$$

Thus

$$
J=\int_{\left|z_{i}\right| \leq \mu_{i}^{1 / 2} /\left(3 \varepsilon^{1 / 2} p^{2 \kappa}\right)} a(\varepsilon y) \eta_{i 2}^{2} Z_{i 0}^{2}\left[\frac{8 \mu_{i}^{2}}{\left(\mu_{i}^{2}+\left|y-\xi_{i}^{\prime}\right|^{2}\right)^{2}}-W_{\xi^{\prime}}\right] d y+O\left(\frac{1}{p \mu_{i}^{2} R}\right) .
$$

Note that in a straightforward but tedious way, by (2.14) we can compute

$$
\int_{\mathbb{R}^{2}} e^{U_{1,0}(z)} Z_{0}^{2}(z)\left(\omega_{1}-U_{1,0}-\frac{1}{2} U_{1,0}^{2}\right)(z) d z=-8 \pi .
$$

Hence by (2.18), (3.2), (3.7), (3.10) and (3.43),

$$
J=\frac{c_{i} a\left(\xi_{i}\right)}{p \mu_{i}^{2}}\left[1+O\left(\frac{1}{R}\right)\right], \quad \forall i=1, \ldots, m .
$$

Next, we estimate $K$. Integrating by parts the first term and the last term of $K$ respectively, we get

$$
\begin{aligned}
K= & -\int_{\Omega_{2}} a(\varepsilon y) Z_{i 0} \nabla \eta_{i 1} \nabla\left(Z_{i 0}-\widehat{Z}_{i 0}\right)+\int_{\Omega_{2}} a(\varepsilon y)\left(Z_{i 0}-\widehat{Z}_{i 0}\right) \nabla \eta_{i 1} \nabla\left(Z_{i 0}-\widehat{Z}_{i 0}\right) \\
& +\int_{\Omega_{2}} a(\varepsilon y)\left(Z_{i 0}-\widehat{Z}_{i 0}\right)^{2}\left|\nabla \eta_{i 1}\right|^{2}+\int_{\Omega_{2}} a(\varepsilon y)\left(Z_{i 0}-\widehat{Z}_{i 0}\right) \nabla \eta_{i 1} \nabla \widehat{Z}_{i 0}+\int_{\Omega_{4}} a(\varepsilon y)\left|\widehat{Z}_{i 0}\right|^{2}\left|\nabla \eta_{i 2}\right|^{2} \\
\equiv & K_{21}+K_{22}+K_{23}+K_{24}+K_{4} .
\end{aligned}
$$

From (2.18), (3.3), (3.7), (3.10), (3.21), (3.22), (3.42), (3.43) and (3.47) we can conclude that

$$
\begin{aligned}
K_{21} & =-\frac{a_{i 0}}{\mu_{i}^{2}} \int_{\left\{\mu_{i} R<\left|z_{i}\right| \leq \mu_{i}(R+1)\right\}} \frac{1}{\left|y-\xi_{i}^{\prime}\right|} a(\varepsilon y) Z_{0}\left(\frac{z_{i}}{\mu_{i}}\right) \eta_{1}^{\prime}\left(\frac{\left|z_{i}\right|}{\mu_{i}}\right)\left[\frac{4}{c_{i}}+o(1)\right] d y \\
& =-\frac{c_{i} a_{i 0}}{4 \mu_{i}} \int_{R}^{R+1} a\left(\xi_{i}\right) \eta_{1}^{\prime}(r)\left[\frac{4}{c_{i}}+O\left(\frac{1}{r^{2}}\right)\right] d r \\
& =\frac{c_{i} a\left(\xi_{i}\right)}{p \mu_{i}^{2}}\left[1+O\left(\frac{1}{R^{2}}\right)\right] .
\end{aligned}
$$

Moreover, by (3.3), (3.7), (3.10), (3.42) and (3.50) we find $\left|\nabla \widehat{Z}_{i 0}\right|=O\left(\frac{1}{\mu_{i}^{2} R^{3}}\right)$ in $\Omega_{2}$. Furthermore,

$$
K_{22}=O\left(\frac{1}{p^{2} \mu_{i}^{2} R}\right), \quad K_{23}=O\left(\frac{1}{p^{2} \mu_{i}^{2} R}\right) \quad \text { and } \quad K_{24}=O\left(\frac{1}{p \mu_{i}^{2} R^{3}}\right) .
$$

By (3.54),

$$
K_{4}=O\left(\frac{|\log d|^{2}}{p^{2} \mu_{i}^{2}}\right)
$$


Combining all these estimates, we conclude

$$
\int_{\Omega_{p}} a(\varepsilon y) \widetilde{Z}_{i 0} \mathcal{L}\left(\widetilde{Z}_{i 0}\right)=J+K=\frac{2 c_{i} a\left(\xi_{i}\right)}{p \mu_{i}^{2}}\left[1+O\left(\frac{1}{R}\right)\right], \quad \forall i=1, \ldots, m .
$$

According to (3.56), we need just to consider $\int_{\Omega_{p}} a(\varepsilon y) \widetilde{Z}_{k 0} \mathcal{L}\left(\widetilde{Z}_{i 0}\right)$ when $k \neq i$. Using the previous estimates of $\mathcal{L}\left(\widetilde{Z}_{i 0}\right)$ and $\widetilde{Z}_{k 0}$, we can easily prove that

$$
\begin{array}{cc}
\int_{\Omega_{1}} a(\varepsilon y) \widetilde{Z}_{k 0} \mathcal{L}\left(\widetilde{Z}_{i 0}\right)=O\left(\frac{R^{2} \log p}{p^{2} \mu_{i} \mu_{k}}\right), & \int_{\Omega_{2}} a(\varepsilon y) \widetilde{Z}_{k 0} \mathcal{L}\left(\widetilde{Z}_{i 0}\right)=O\left(\frac{\log p}{p^{2} \mu_{i} \mu_{k}}\right), \\
\int_{\Omega_{4}} a(\varepsilon y) \widetilde{Z}_{k 0} \mathcal{L}\left(\widetilde{Z}_{i 0}\right)=O\left(\frac{|\log d|^{2}}{p^{2} \mu_{i} \mu_{k}}\right), & \int_{\Omega_{3, i} \cup \widetilde{\Omega}_{3}} a(\varepsilon y) \widetilde{Z}_{k 0} \mathcal{L}\left(\widetilde{Z}_{i 0}\right)=O\left(\frac{\log p}{p^{2} \mu_{i} \mu_{k}}\right),
\end{array}
$$

and

$$
\int_{\Omega_{3, l}} a(\varepsilon y) \widetilde{Z}_{k 0} \mathcal{L}\left(\widetilde{Z}_{i 0}\right)=O\left(\frac{\log ^{2} p}{p^{2} \mu_{i} \mu_{k}}\right) \quad \text { for all } l \neq i \text { and } l \neq k .
$$

It remains to calculate the integral over $\Omega_{3, k}$. From (3.27) and an integration by parts we get

$$
\int_{\Omega_{3, k}} a(\varepsilon y) \widetilde{Z}_{k 0} \mathcal{L}\left(\widetilde{Z}_{i 0}\right)=\int_{\Omega_{3, k}} a(\varepsilon y) \widetilde{Z}_{i 0} \mathcal{L}\left(\widetilde{Z}_{k 0}\right)-\int_{\partial \Omega_{3, k}} a(\varepsilon y) \widehat{Z}_{k 0} \frac{\partial \widehat{Z}_{i 0}}{\partial \nu}+\int_{\partial \Omega_{3, k}} a(\varepsilon y) \widehat{Z}_{i 0} \frac{\partial \widehat{Z}_{k 0}}{\partial \nu} .
$$

As above, we know that

$$
\int_{\Omega_{3, k}} a(\varepsilon y) \widetilde{Z}_{i 0} \mathcal{L}\left(\widetilde{Z}_{k 0}\right)=O\left(\frac{\log p}{p^{2} \mu_{i} \mu_{k}}\right) .
$$

On $\partial \Omega_{3, k}$, by (2.7) and (3.24) we have

$$
\widehat{Z}_{k 0}=O\left(\frac{1}{\mu_{k}}\right), \quad \widehat{Z}_{i 0}=O\left(\frac{\log p}{p \mu_{i}}\right),
$$

and

So

$$
\left|\nabla \widehat{Z}_{k 0}\right|=O\left(\frac{\varepsilon^{1 / 2} p^{2 \kappa-1}}{\mu_{k}^{3 / 2}}\right), \quad \quad\left|\nabla \widehat{Z}_{i 0}\right|=O\left(\frac{\varepsilon p^{\kappa-1}}{\mu_{i}}\right) .
$$

By the above estimates, we obtain

$$
\int_{\Omega_{3, k}} a(\varepsilon y) \widetilde{Z}_{k 0} \mathcal{L}\left(\widetilde{Z}_{i 0}\right)=O\left(\frac{\log p}{p^{2} \mu_{i} \mu_{k}}\right) .
$$

$$
\int_{\Omega_{p}} a(\varepsilon y) \widetilde{Z}_{k 0} \mathcal{L}\left(\widetilde{Z}_{i 0}\right)=O\left(\frac{\log ^{2} p}{p^{2} \mu_{i} \mu_{k}}\right), \quad \text { if } i \neq k .
$$

As a consequence, replacing (3.57) and (3.58) to (3.56) we get

$$
\frac{\left|d_{i}\right|}{\mu_{i}} \leq C p\|h\|_{*}+C \sum_{k=1}^{m} \frac{\left|d_{k}\right|}{\mu_{k}} \frac{\log ^{2} p}{p}
$$

Using linear algebra arguments, we then prove Claim 2 for $d_{i}$ and complete the proof by (3.33).

Step 4: Proof of Proposition 3.3. We first establish the validity of the a priori estimate

$$
\|\phi\|_{L^{\infty}\left(\Omega_{p}\right)} \leq C p\|h\|_{*}
$$

for any $\phi, c_{i j}$ solutions of problem (3.6) and any $h \in C^{0, \alpha}\left(\bar{\Omega}_{p}\right)$. Step 3 gives

$$
\|\phi\|_{L^{\infty}\left(\Omega_{p}\right)} \leq C p\left(\|h\|_{*}+\sum_{i=1}^{m} \sum_{j=1}^{J_{i}}\left|c_{i j}\right| \cdot\left\|\chi_{i} Z_{i j}\right\|_{*}\right) \leq C p\left(\|h\|_{*}+\sum_{i=1}^{m} \sum_{j=1}^{J_{i}} \mu_{i}\left|c_{i j}\right|\right) .
$$


As before, arguing by contradiction to (3.59), we can proceed as in Step 2 and suppose further that

$$
\left\|\phi_{n}\right\|_{L^{\infty}\left(\Omega_{p_{n}}\right)}=1, \quad p_{n}\left\|h_{n}\right\|_{*} \rightarrow 0, \quad p_{n} \sum_{i=1}^{m} \sum_{j=1}^{J_{i}} \mu_{i}^{n}\left|c_{i j}^{n}\right| \geq \delta>0 \quad \text { as } n \rightarrow+\infty .
$$

We omit the dependence on $n$. It suffices to estimate the values of the constants $c_{i j}$. For this aim, let us consider the cut-off function $\eta_{i 2}$ given by (3.25)-(3.26). For any $i=1, \ldots, m$ and $j=1, J_{i}$, multiplying (3.6) by $a(\varepsilon y) \eta_{i 2} Z_{i j}$ and integrating by parts we find

$$
\int_{\Omega_{p}} a(\varepsilon y) \phi \mathcal{L}\left(\eta_{i 2} Z_{i j}\right)=\int_{\Omega_{p}} a(\varepsilon y) h \eta_{i 2} Z_{i j}+\sum_{k=1}^{m} \sum_{t=1}^{J_{k}} c_{k t} \int_{\Omega_{p}} \chi_{k} Z_{k t} \eta_{i 2} Z_{i j} .
$$

Notice that

$$
\begin{aligned}
\mathcal{L}\left(\eta_{i 2} Z_{i j}\right) & =\eta_{i 2} \mathcal{L}\left(Z_{i j}\right)-Z_{i j} \Delta_{a(\varepsilon y)} \eta_{i 2}-2 \nabla \eta_{i 2} \nabla Z_{i j} \\
& =\eta_{i 2}\left[-\Delta_{a(\varepsilon y)} Z_{i j}-\frac{8 \mu_{i}^{2}}{\left(\mu_{i}^{2}+\left|y-\xi_{i}^{\prime}\right|^{2}\right)^{2}} Z_{i j}\right]+\left[\frac{8 \mu_{i}^{2}}{\left(\mu_{i}^{2}+\left|y-\xi_{i}^{\prime}\right|^{2}\right)^{2}}-W_{\xi^{\prime}}\right] \eta_{i 2} Z_{i j}-\varepsilon^{2} \eta_{i 2} Z_{i j}+O\left(\varepsilon^{3} / d^{3}\right) \\
& \equiv B_{1}+B_{2}-\varepsilon^{2} \eta_{i 2} Z_{i j}+O\left(\varepsilon^{3} / d^{3}\right) .
\end{aligned}
$$

By (3.3), (3.7), (3.10), (3.42) and (3.43) we can compute

$$
B_{1}=O\left(\frac{\varepsilon}{\left(\left|y-\xi_{i}^{\prime}\right|+\mu_{i}\right)^{2}}\right) .
$$

To estimate $B_{2}$, we decompose $\operatorname{supp}\left(\eta_{i 2}\right)$ into several regions:

$$
\begin{gathered}
\widehat{\Omega}_{1 k}=\left\{y \in \Omega_{p}|| z_{k} \mid \leq \frac{\mu_{k}^{1 / 2}}{3 \varepsilon^{1 / 2} p^{2 \kappa}}\right\}, \quad \forall k=1, \ldots, m, \\
\widehat{\Omega}_{2}=\left\{y \in \Omega_{p}|| z_{i}\left|\leq \frac{6 d}{\varepsilon}, \quad\right| z_{k} \mid>\frac{\mu_{k}^{1 / 2}}{3 \varepsilon^{1 / 2} p^{2 \kappa}}, \quad k=1, \ldots, m\right\},
\end{gathered}
$$

where $z_{k}=y-\xi_{k}^{\prime}$ for $k=1, \ldots, l$, but $z_{k}=F_{k}^{p}(y)$ for $k=l+1, \ldots, m$. Note that, by (2.7) and (3.43),

$$
\left|y-\xi_{i}^{\prime}\right| \geq\left|\xi_{i}^{\prime}-\xi_{k}^{\prime}\right|-\left|y-\xi_{k}^{\prime}\right| \geq\left|\xi_{i}^{\prime}-\xi_{k}^{\prime}\right|-\frac{\mu_{k}^{1 / 2}}{\varepsilon^{1 / 2} p^{2 \kappa}} \geq \frac{1}{\varepsilon p^{\kappa}}\left[1-\frac{\left(\varepsilon \mu_{k}\right)^{1 / 2}}{p^{\kappa}}\right],
$$

uniformly in $\widehat{\Omega}_{1 k}, k \neq i$. In $\widehat{\Omega}_{1 i}$, by (3.2), (3.7) and (3.10) we have that for any $i=1, \ldots, l$ and $j=1,2$,

$$
B_{2}=-\frac{8 \mu_{i}^{2}\left(y-\xi_{i}^{\prime}\right)_{j}}{\left(\mu_{i}^{2}+\left|y-\xi_{i}^{\prime}\right|^{2}\right)^{3}}\left\{\frac{1}{p}\left(\omega_{1}-U_{1,0}-\frac{1}{2} U_{1,0}^{2}\right)\left(\frac{y-\xi_{i}^{\prime}}{\mu_{i}}\right)+O\left(\frac{\log ^{4}\left(\left|\frac{y-\xi_{i}^{\prime}}{\mu_{i}}\right|+2\right)}{p^{2}}\right)\right\},
$$

and for any $i=l+1, \ldots, m$ and $j=J_{i}=1$,

$$
B_{2}=-\frac{8 \mu_{i}^{2}}{\left(\mu_{i}^{2}+\left|y-\xi_{i}^{\prime}\right|^{2}\right)^{2}} \frac{\left(F_{i}^{p}(y)\right)_{1}}{\mu_{i}^{2}+\left|F_{i}^{p}(y)\right|^{2}}\left\{\frac{1}{p}\left(\omega_{1}-U_{1,0}-\frac{1}{2} U_{1,0}^{2}\right)\left(\frac{y-\xi_{i}^{\prime}}{\mu_{i}}\right)+O\left(\frac{\log ^{4}\left(\left|\frac{y-\xi_{i}^{\prime}}{\mu_{i}}\right|+2\right)}{p^{2}}\right)\right\} .
$$

In $\widehat{\Omega}_{1 k}, k \neq i$, by (3.2), (3.7), (3.10), (3.43) and (3.62),

$$
B_{2}=O\left(\frac{8 \varepsilon p^{\kappa} \mu_{k}^{2}}{\left(\mu_{k}^{2}+\left|y-\xi_{k}^{\prime}\right|^{2}\right)^{2}}\right) .
$$

In $\widehat{\Omega}_{2}$, by (3.1),

$$
B_{2}=\sum_{k=1}^{m} O\left(\frac{\mu_{k}^{2}}{\left|y-\xi_{k}^{\prime}\right|^{4}} \cdot \frac{\varepsilon^{1 / 2} p^{2 \kappa}}{\mu_{i}^{1 / 2}}\right)
$$


Hence by (2.6), (2.13) and (3.43),

$$
\int_{\Omega_{p}} a(\varepsilon y) \phi \mathcal{L}\left(\eta_{i 2} Z_{i j}\right)=-\frac{1}{p \mu_{i}} a\left(\xi_{i}\right) E_{j}\left(\widehat{\phi}_{i}\right)+O\left(\frac{1}{p^{2} \mu_{i}}\|\phi\|_{L^{\infty}\left(\Omega_{p}\right)}\right),
$$

where for any $i=1, \ldots, l$ and $j=1,2, \widehat{\phi}_{i}(z)=\phi\left(\xi_{i}^{\prime}+\mu_{i} z\right)$ and

$$
E_{j}\left(\widehat{\phi}_{i}\right)=\int_{B \frac{1}{3 \varepsilon^{1 / 2} \mu_{i}^{1 / 2} p^{2 \kappa}}(0)} \frac{8 z_{j}}{\left(|z|^{2}+1\right)^{3}}\left(\omega_{1}-U_{1,0}-\frac{1}{2} U_{1,0}^{2}\right)(|z|) \widehat{\phi}_{i}(z) d z,
$$

but for any $i=l+1, \ldots, m$ and $j=1, \widehat{\phi}_{i}(z)=\phi\left(\left(F_{i}^{p}\right)^{-1}\left(\mu_{i} z\right)\right)$ and

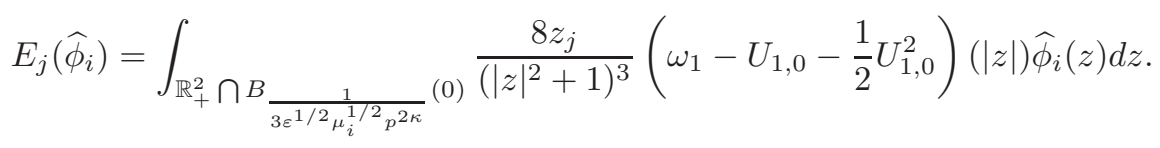

On the other hand, since $\left\|\eta_{i 2} Z_{i j}\right\|_{L^{\infty}\left(\Omega_{p}\right)} \leq C \mu_{i}^{-1}$, we obtain

$$
\int_{\Omega_{p}} a(\varepsilon y) h \eta_{i 2} Z_{i j}=O\left(\frac{1}{\mu_{i}}\|h\|_{*}\right) .
$$

Moreover, if $1 \leq k=i \leq l$, by (3.3) and (3.7),

$$
\int_{\Omega_{p}} \chi_{k} Z_{k t} \eta_{i 2} Z_{i j}=\int_{\mathbb{R}^{2}} \chi Z_{t} Z_{j} d z=D_{t} \delta_{t j}
$$

and if $l+1 \leq k=i \leq m$, by (3.3), (3.9) and (3.10),

$$
\int_{\Omega_{p}} \chi_{k} Z_{k 1} \eta_{i 2} Z_{i 1}=\int_{\mathbb{R}_{+}^{2}} \chi Z_{1}^{2}\left[1+O\left(\varepsilon \mu_{i}|z|\right)\right] d z=\frac{1}{2} D_{1}\left[1+O\left(\varepsilon \mu_{i}\right)\right],
$$

and if $k \neq i$, by $(3.62)$,

$$
\int_{\Omega_{p}} \chi_{k} Z_{k t} \eta_{i 2} Z_{i j}=O\left(\mu_{k} \varepsilon p^{\kappa}\right)
$$

Inserting estimates (3.63)-(3.67) into (3.61), we deduce that for any $i=1, \ldots, m$ and $j=1, J_{i}$,

$$
D_{j} c_{i j}+O\left(\sum_{k \neq i}^{m} \sum_{t=1}^{J_{k}} \mu_{k} \varepsilon p^{\kappa}\left|c_{k t}\right|\right)=O\left(\frac{1}{\mu_{i}}\|h\|_{*}+\frac{1}{p \mu_{i}}\|\phi\|_{L^{\infty}\left(\Omega_{p}\right)}\right) .
$$

Furthermore,

$$
\sum_{i=1}^{m} \sum_{j=1}^{J_{i}} \mu_{i}\left|c_{i j}\right|=O\left(\|h\|_{*}+\frac{1}{p}\|\phi\|_{L^{\infty}\left(\Omega_{p}\right)}\right)
$$

Since $\sum_{i=1}^{m} \sum_{j=1}^{J_{i}} \mu_{i}\left|c_{i j}\right|=o(1)$, as in contradiction arguments of Step 2, we conclude that for any $i=1, \ldots, l$,

$$
\widehat{\phi}_{i} \rightarrow C_{i} \frac{|z|^{2}-1}{|z|^{2}+1} \quad \text { uniformly in } C_{l o c}^{0}\left(\mathbb{R}^{2}\right)
$$

but for any $i=l+1, \ldots, m$,

$$
\widehat{\phi}_{i} \rightarrow C_{i} \frac{|z|^{2}-1}{|z|^{2}+1} \quad \text { uniformly in } C_{l o c}^{0}\left(\mathbb{R}_{+}^{2}\right)
$$

with some constant $C_{i} \in \mathbb{R}$. Hence in (3.63), we have a better estimate, since by Lebesgue's theorem we can derive that for any $i=1, \ldots, l$ and $j=1,2$,

$$
E_{j}\left(\widehat{\phi}_{i}\right) \longrightarrow C_{i} \int_{\mathbb{R}^{2}} \frac{8 z_{j}}{\left(|z|^{2}+1\right)^{3}} \frac{|z|^{2}-1}{|z|^{2}+1}\left(\omega_{1}-U_{1,0}-\frac{1}{2} U_{1,0}^{2}\right)(|z|) d z=0,
$$


and for any $i=l+1, \ldots, m$ and $j=1$,

$$
E_{j}\left(\widehat{\phi}_{i}\right) \longrightarrow C_{i} \int_{\mathbb{R}_{+}^{2}} \frac{8 z_{j}}{\left(|z|^{2}+1\right)^{3}} \frac{|z|^{2}-1}{|z|^{2}+1}\left(\omega_{1}-U_{1,0}-\frac{1}{2} U_{1,0}^{2}\right)(|z|) d z=0 .
$$

Therefore,

$$
\sum_{i=1}^{m} \sum_{j=1}^{J_{i}} \mu_{i}\left|c_{i j}\right|=o\left(\frac{1}{p}\right)+O\left(\|h\|_{*}\right),
$$

which is impossible because of (3.60). So estimate (3.59) is established and then by (3.68), we obtain

$$
\left|c_{i j}\right| \leq C \frac{1}{\mu_{i}}\|h\|_{*} .
$$

Now consider the Hilbert space

$$
H_{\xi}=\left\{\phi \in H^{1}\left(\Omega_{p}\right) \mid \int_{\Omega_{p}} \chi_{i} Z_{i j} \phi=0 \quad \text { for any } i=1, \ldots, m, j=1, J_{i} ; \quad \frac{\partial \phi}{\partial \nu}=0 \quad \text { on } \partial \Omega_{p}\right\}
$$

with the norm $\|\phi\|_{H^{1}\left(\Omega_{p}\right)}^{2}=\int_{\Omega_{p}} a(\varepsilon y)\left(|\nabla \phi|^{2}+\varepsilon^{2} \phi^{2}\right)$. Equation (3.6) is equivalent to find $\phi \in H_{\xi}$ such that

$$
\int_{\Omega_{p}} a(\varepsilon y)\left(\nabla \phi \nabla \psi+\varepsilon^{2} \phi \psi\right)-\int_{\Omega_{p}} a(\varepsilon y) W_{\xi^{\prime}} \phi \psi=\int_{\Omega_{p}} a(\varepsilon y) h \psi \quad \forall \psi \in H_{\xi} .
$$

By Fredholm's alternative this is equivalent to the uniqueness of solutions to this problem, which is guaranteed by estimate (3.59). Finally, for $p \geq p_{m}$ fixed, by density of $C^{0, \alpha}\left(\bar{\Omega}_{p}\right)$ in $\left(C\left(\bar{\Omega}_{p}\right)\right.$, $\left.\|\cdot\|_{L^{\infty}\left(\Omega_{p}\right)}\right)$, we can approximate $h \in C\left(\bar{\Omega}_{p}\right)$ by smooth functions and, by (3.59) and elliptic regularity theory, we find that for any $h \in C\left(\bar{\Omega}_{p}\right)$, problem (3.6) admits a unique solution which belongs to $H^{2}\left(\Omega_{p}\right)$ and satisfies the a priori estimate (3.12). The proof is complete.

Remark 3.7. Given $h \in C\left(\bar{\Omega}_{p}\right)$ with $\|h\|_{*}<\infty$, let $\phi$ be the solution to (3.6) given by Proposition 3.3. Testing the first equation of (3.6) against $a(\varepsilon y) \phi$, we get

$$
\|\phi\|_{H^{1}\left(\Omega_{p}\right)}^{2}=\int_{\Omega_{p}} a(\varepsilon y) W_{\xi^{\prime}} \phi^{2}+\int_{\Omega_{p}} a(\varepsilon y) h \phi .
$$

Furthermore, by (3.1) we obtain

$$
\|\phi\|_{H^{1}\left(\Omega_{p}\right)} \leq C\left(\|h\|_{*}+\|\phi\|_{L^{\infty}\left(\Omega_{p}\right)}\right) .
$$

\section{THE NONLINEAR PROBLEM}

In order to solve problem (2.29) we first consider an auxiliary nonlinear problem: for any points $\xi=\left(\xi_{1}, \ldots, \xi_{m}\right) \in$ $\mathcal{O}_{p}$, we find a function $\phi$ and scalars $c_{i j}, i=1, \ldots, m, j=1, J_{i}$ such that

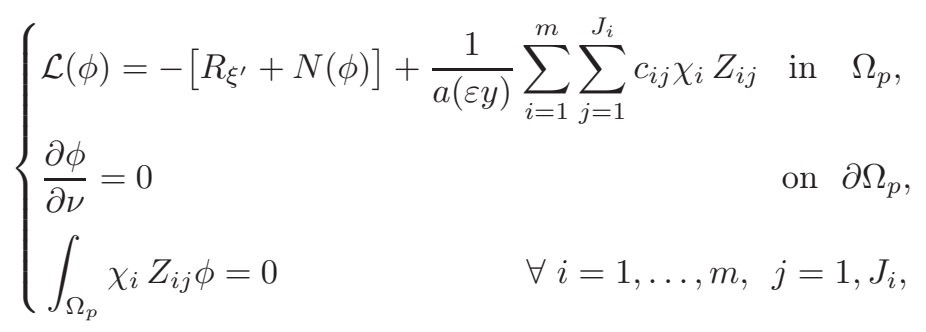

where $W_{\xi^{\prime}}=p V_{\xi^{\prime}}^{p-1}$ satisfies (3.1)-(3.2), and $R_{\xi^{\prime}}, N(\phi)$ are given by (2.28). 
Proposition 4.1. Let $m$ be a positive integer. Then there exist constants $C>0$ and $p_{m}>1$ such that for any $p>p_{m}$ and any points $\xi=\left(\xi_{1}, \ldots, \xi_{m}\right) \in \mathcal{O}_{p}$, problem (4.1) admits a unique solution $\phi_{\xi^{\prime}}$ for some coefficients $c_{i j}\left(\xi^{\prime}\right), i=1, \ldots, m, j=1, J_{i}$, such that

$$
\left\|\phi_{\xi^{\prime}}\right\|_{L^{\infty}\left(\Omega_{p}\right)} \leq \frac{C}{p^{3}}, \quad \sum_{i=1}^{m} \sum_{j=1}^{J_{i}} \mu_{i}\left|c_{i j}\left(\xi^{\prime}\right)\right| \leq \frac{C}{p^{4}} \quad \text { and } \quad\left\|\phi_{\xi^{\prime}}\right\|_{H^{1}\left(\Omega_{p}\right)} \leq \frac{C}{p^{3}} .
$$

Furthermore, the map $\xi^{\prime} \mapsto \phi_{\xi^{\prime}}$ is a $C^{1}$-function in $C\left(\bar{\Omega}_{p}\right)$ and $H^{1}\left(\Omega_{p}\right)$.

Proof. Proposition 3.3, Remarks 3.2 and 3.7 allow us to apply the Contraction Mapping Theorem and the Implicit Function Theorem to find a solution for problem (4.1) satisfying (4.2) and the corresponding regularity of the map $\xi^{\prime} \mapsto \phi_{\xi^{\prime}}$. Since it is a standard procedure, we omit the detailed proof here, see Lemma 4.1 in [22] for a similar proof.

Remark 4.2. The function $V_{\xi^{\prime}}+\phi_{\xi^{\prime}}$, where $\phi_{\xi^{\prime}}$ is given by Proposition 4.1, is positive in $\bar{\Omega}_{p}$. In fact, we observe first that $p^{2} \phi_{\xi^{\prime}} \rightarrow 0$ uniformly in $C\left(\bar{\Omega}_{p}\right)$. Furthermore, from Remark 2.6 and the definition of $V_{\xi^{\prime}}$ in $(2.27)$ we have that, in each region $\left|y-\xi_{i}^{\prime}\right|<1 /\left(\varepsilon p^{2 \kappa}\right), V_{\xi^{\prime}}+\phi_{\xi^{\prime}}$ is positive. Outside these regions, by (2.20) and (2.27) we may get the same result.

\section{VARIATIONAL REDUCTION}

After problem (4.1) has been solved, we find a solution of problem (2.29) and hence to the original problem (1.5) if we find $\xi^{\prime}$ such that the coefficient $c_{i j}\left(\xi^{\prime}\right)$ in (4.1) satisfies

$$
c_{i j}\left(\xi^{\prime}\right)=0 \quad \text { for all } i=1, \ldots, m, j=1, J_{i} .
$$

Equation (1.5) is the Euler-Lagrange equation of the energy functional $J_{p}$ given by

$$
J_{p}(u)=\frac{1}{2} \int_{\Omega} a(x)\left(|\nabla u|^{2}+u^{2}\right) d x-\frac{1}{p+1} \int_{\Omega} a(x) u_{+}^{p+1} d x, \quad u \in H^{1}(\Omega) .
$$

We define

$$
F_{p}(\xi)=J_{p}\left(U_{\xi}+\widetilde{\phi}_{\xi}\right) \quad \forall \xi \in \mathcal{O}_{p},
$$

where $U_{\xi}$ is the function defined in (2.16) and

$$
\widetilde{\phi}_{\xi}(x)=\varepsilon^{-2 /(p-1)} \phi_{\xi^{\prime}}\left(\varepsilon^{-1} x\right), \quad x \in \Omega,
$$

with $\phi_{\xi^{\prime}}$ the unique solution to problem (4.1) given by Proposition 4.1. Critical points of $F_{p}$ correspond to solutions of (5.1) for large $p$, as the following results states.

Proposition 5.1. The function $F_{p}: \mathcal{O}_{p} \mapsto \mathbb{R}$ is of class $C^{1}$. Moreover, for all $p$ sufficiently large, if $D_{\xi} F_{p}(\xi)=0$, then $\xi^{\prime}=\xi / \varepsilon$ satisfies (5.1).

Proof. A direct consequence of the results obtained in Proposition 4.1 and the definition of function $U_{\xi}$ is the fact that $F_{p}(\xi)$ is a $C^{1}$-function of $\xi$ in $\mathcal{O}_{p}$ since the map $\xi \mapsto \widetilde{\phi}_{\xi}$ is a $C^{1}$-map into $H^{1}(\Omega)$. Recall that

$$
I_{p}(v)=\frac{1}{2} \int_{\Omega_{p}} a(\varepsilon y)\left(|\nabla v|^{2}+\varepsilon^{2} v^{2}\right) d y-\frac{1}{p+1} \int_{\Omega_{p}} a(\varepsilon y) v_{+}^{p+1} d y, \quad v \in H^{1}\left(\Omega_{p}\right),
$$

Then, making a change of variable, we get

$$
F_{p}(\xi)=J_{p}\left(U_{\xi}+\widetilde{\phi}_{\xi}\right)=\varepsilon^{-4 /(p-1)} I_{p}\left(V_{\xi^{\prime}}+\phi_{\xi^{\prime}}\right) .
$$


Since $\phi_{\xi^{\prime}}$ solves equation (4.1) and $D_{\xi} F_{p}(\xi)=0$, we have that for any $k=1, \ldots, m$ and $t=1, J_{k}$,

$$
\begin{aligned}
0 & =I_{p}^{\prime}\left(V_{\xi^{\prime}}+\phi_{\xi^{\prime}}\right) \partial_{\left(\xi_{k}^{\prime}\right)_{t}}\left(V_{\xi^{\prime}}+\phi_{\xi^{\prime}}\right) \\
& =\int_{\Omega_{p}} a(x)\left[-\Delta_{a(\varepsilon y)}\left(V_{\xi^{\prime}}+\phi_{\xi^{\prime}}\right)+\varepsilon^{2}\left(V_{\xi^{\prime}}+\phi_{\xi^{\prime}}\right)-\left(V_{\xi^{\prime}}+\phi_{\xi^{\prime}}\right)^{p}\right] \partial_{\left(\xi_{k}^{\prime}\right)_{t}}\left(V_{\xi^{\prime}}+\phi_{\xi^{\prime}}\right) \\
& =\sum_{i=1}^{m} \sum_{j=1}^{J_{i}} c_{i j}\left(\xi^{\prime}\right) \int_{\Omega_{p}} \chi_{i} Z_{i j} \partial_{\left(\xi_{k}^{\prime}\right)_{t}}\left(V_{\xi^{\prime}}+\phi_{\xi^{\prime}}\right) \\
& =\sum_{i=1}^{m} \sum_{j=1}^{J_{i}} c_{i j}\left(\xi^{\prime}\right) \int_{\Omega_{p}} \chi_{i} Z_{i j} \partial_{\left(\xi_{k}^{\prime}\right)_{t}} V_{\xi^{\prime}}-\sum_{i=1}^{m} \sum_{j=1}^{J_{i}} c_{i j}\left(\xi^{\prime}\right) \int_{\Omega_{p}} \phi_{\xi^{\prime}} \partial_{\left(\xi_{k}^{\prime}\right)_{t}}\left(\chi_{i} Z_{i j}\right),
\end{aligned}
$$

because $\int_{\Omega_{p}} \chi_{i} Z_{i j} \phi_{\xi^{\prime}}=0$. Notice first that by (3.3), (3.7), (3.10) and (3.43), a direct computation shows

$$
\left|\partial_{\left(\xi_{k}^{\prime}\right)_{t}}\left(\chi_{i} Z_{i j}\right)\right|=O\left(\frac{1}{\mu_{i}}\left|\partial_{\left(\xi_{k}^{\prime}\right)_{t}} \log \mu_{i}\right|+\frac{1}{\mu_{i}^{2}} \delta_{k i}\right) .
$$

On the other hand, since $D_{\xi^{\prime}} V_{\xi^{\prime}}(y)=\varepsilon^{2 /(p-1)} D_{\xi^{\prime}} U_{\xi}(\varepsilon y)$, by (2.9), (2.10), (2.16), (2.20) and (2.21) we obtain

$$
\begin{aligned}
\partial_{\left(\xi_{k}^{\prime}\right)_{t}} V_{\xi^{\prime}}(y)= & \sum_{i=1}^{m} \frac{\varepsilon^{2 /(p-1)}}{\gamma \mu_{i}^{2 /(p-1)}}\left\{\partial_{\left(\xi_{k}^{\prime}\right)_{t}}\left[U_{\delta_{i}, \xi_{i}}(\varepsilon y)+\frac{1}{p} \omega_{1}\left(\frac{y-\xi_{i}^{\prime}}{\mu_{i}}\right)+\frac{1}{p^{2}} \omega_{2}\left(\frac{y-\xi_{i}^{\prime}}{\mu_{i}}\right)+\gamma \mu_{i}^{2 /(p-1)} H_{i}(\varepsilon y)\right]\right. \\
& \left.-\frac{2 \partial_{\left(\xi_{k}^{\prime}\right)_{t}} \log \mu_{i}}{p-1}[p+O(1)]\right\} .
\end{aligned}
$$

By (2.6) and (3.3),

$$
\begin{aligned}
\partial_{\left(\xi_{k}^{\prime}\right)_{t}} U_{\delta_{i}, \xi_{i}}(\varepsilon y) & =\frac{4}{\mu_{i}} \delta_{k i} Z_{t}\left(\frac{y-\xi_{i}^{\prime}}{\mu_{i}}\right)+2 Z_{0}\left(\frac{y-\xi_{i}^{\prime}}{\mu_{i}}\right) \partial_{\left(\xi_{k}^{\prime}\right)_{t}} \log \mu_{i} \\
& =\frac{4}{\mu_{i}} \delta_{k i} Z_{t}\left(\frac{y-\xi_{i}^{\prime}}{\mu_{i}}\right)+2\left(1-\frac{2 \mu_{i}^{2}}{\left|y-\xi_{i}^{\prime}\right|^{2}+\mu_{i}^{2}}\right) \partial_{\left(\xi_{k}^{\prime}\right)_{t}} \log \mu_{i},
\end{aligned}
$$

while for $j=1,2$, by (2.13) and (3.3),

$$
\begin{aligned}
\partial_{\left(\xi_{k}^{\prime}\right)_{t}} \omega_{j}\left(\frac{y-\xi_{i}^{\prime}}{\mu_{i}}\right) & =-\omega_{j}^{\prime}\left(\frac{\left|y-\xi_{i}^{\prime}\right|}{\mu_{i}}\right)\left\{\frac{\left(y-\xi_{i}^{\prime}\right)_{t}}{\left|y-\xi_{i}^{\prime}\right|} \frac{1}{\mu_{i}} \delta_{k i}+\frac{\left|y-\xi_{i}^{\prime}\right|}{\mu_{i}} \partial_{\left(\xi_{k}^{\prime}\right)_{t}} \log \mu_{i}\right\} \\
& =-\frac{1}{\mu_{i}} \delta_{k i}\left[C_{j} Z_{t}\left(\frac{y-\xi_{i}^{\prime}}{\mu_{i}}\right)+O\left(\frac{\mu_{i}^{2}}{\left|y-\xi_{i}^{\prime}\right|^{2}+\mu_{i}^{2}}\right)\right]-C_{j}\left[1+O\left(\frac{\mu_{i}^{2}}{\left|y-\xi_{i}^{\prime}\right|^{2}+\mu_{i}^{2}}\right)\right] \partial_{\left(\xi_{k}^{\prime}\right)_{t}} \log \mu_{i} .
\end{aligned}
$$

Additionally, as in the proof of Lemma 2.1, we can prove that

$$
\begin{aligned}
\partial_{\left(\xi_{k}^{\prime}\right)_{t}\left[\gamma \mu_{i}^{2 /(p-1)} H_{i}(\varepsilon y)\right]=} & \delta_{k i}\left(1-\frac{C_{1}}{4 p}-\frac{C_{2}}{4 p^{2}}\right) c_{i} \partial_{\left(\xi_{k}^{\prime}\right)_{t}} H\left(\varepsilon y, \xi_{i}\right)+\left(-2+\frac{C_{1}}{p}+\frac{C_{2}}{p^{2}}\right) \partial_{\left(\xi_{k}^{\prime}\right)_{t}} \log \mu_{i} \\
& +O\left(\frac{1}{p}\left|\partial_{\left(\xi_{k}^{\prime}\right)_{t}} \log \mu_{i}\right|\right)
\end{aligned}
$$

Thus by (2.9) and (2.23),

$$
\begin{aligned}
\partial_{\left(\xi_{k}^{\prime}\right)_{t}} V_{\xi^{\prime}}(y)= & \frac{1}{p^{p /(p-1)} \mu_{k}^{(p+1) /(p-1)}}\left\{\left(1-\frac{C_{1}}{4 p}-\frac{C_{2}}{4 p^{2}}\right)\left[4 Z_{t}\left(\frac{y-\xi_{k}^{\prime}}{\mu_{k}}\right)+c_{k} \mu_{k} \partial_{\left(\xi_{k}^{\prime}\right)_{t}} H\left(\varepsilon y, \xi_{k}\right)\right]\right. \\
& \left.+O\left(\frac{1}{p} \frac{\mu_{k}^{2}}{\left|y-\xi_{k}^{\prime}\right|^{2}+\mu_{k}^{2}}\right)\right\}+O\left(\frac{1}{p} \sum_{i=1}^{m}\left|\partial_{\left(\xi_{k}^{\prime}\right)_{t}} \log \mu_{i}\right|\right) .
\end{aligned}
$$


Hence for each $k=1, \ldots, m$ and $t=1, J_{k},(5.4)$ can be written as

$$
\begin{aligned}
& \sum_{i, j} \frac{c_{i j}\left(\xi^{\prime}\right)}{p^{p /(p-1)} \mu_{k}^{2 /(p-1)}}\left\{\delta_{k i}\left[\frac{c_{i}}{2 \pi} \int_{\mathbb{R}^{2}} \chi(|z|) Z_{j}(z) Z_{t}(z) d z+O\left(\frac{1}{p}\right)\right]+\left(1-\delta_{k i}\right) O\left(\frac{\mu_{i}}{\left|\xi_{i}^{\prime}-\xi_{k}^{\prime}\right|}\right)\right\} \\
& +\sum_{i j}\left|c_{i j}\left(\xi^{\prime}\right)\right|\left\{O\left(\frac{1}{p} \mu_{i} \sum_{s=1}^{m}\left|\partial_{\left(\xi_{k}^{\prime}\right)_{t}} \log \mu_{s}\right|\right)+\left\|\phi_{\xi^{\prime}}\right\|_{L^{\infty}\left(\Omega_{p}\right)} O\left(\mu_{i}\left|\partial_{\left(\xi_{k}^{\prime}\right)_{t}} \log \mu_{i}\right|+\delta_{k i}\right)\right\}=0
\end{aligned}
$$

and then, by (2.7), (2.23), (2.24) and (4.2),

$$
\frac{c_{k} c_{k t}\left(\xi^{\prime}\right)}{p^{p /(p-1)} \mu_{k}^{2 /(p-1)}} \int_{0}^{R_{0}+1} \chi(r) \frac{r^{3}}{\left(1+r^{2}\right)^{2}} d r+\sum_{i=1}^{m} \sum_{j=1}^{J_{i}}\left|c_{i j}\left(\xi^{\prime}\right)\right| O\left(\frac{\delta_{k i}}{p^{2} \mu_{k}^{2 /(p-1)}}+\varepsilon \mu_{i} p^{\kappa-1}\right)=0,
$$

which implies $c_{k t}\left(\xi^{\prime}\right)=0$ for each $k=1, \ldots, m$ and $t=1, J_{k}$.

\section{EXPANSION OF THE ENERGY}

Proposition 6.1. Let $m$ be a positive integer. With the choice of $\mu_{i}$ 's given by (2.22), there exists $p_{m}>1$ such that for any $p>p_{m}$ and any points $\xi=\left(\xi_{1}, \ldots, \xi_{m}\right) \in \mathcal{O}_{p}$, the following expansion holds

$$
F_{p}(\xi)=\frac{e}{2 p} \sum_{i=1}^{m} c_{i} a\left(\xi_{i}\right)\left[1-\frac{2 \log p}{p}+\frac{\mathcal{K}+2}{p}-\frac{1}{p} c_{i} H\left(\xi_{i}, \xi_{i}\right)-\frac{1}{p} \sum_{k \neq i}^{m} c_{k} G\left(\xi_{i}, \xi_{k}\right)\right]+O\left(\frac{\log ^{2} p}{p^{3}}\right),
$$

where

$$
\mathcal{K}=\frac{1}{8 \pi} \int_{\mathbb{R}^{2}}\left[\frac{8}{\left(1+|z|^{2}\right)^{2}} U_{1,0}(z)-\Delta \omega_{1}(z)\right] d z
$$

Proof. Multiplying the first equation in (4.1) by $a(\varepsilon y)\left(V_{\xi^{\prime}}+\phi_{\xi^{\prime}}\right)$ and integrating by parts, we obtain

$$
\begin{aligned}
\int_{\Omega_{p}} a(\varepsilon y)\left[\left|\nabla\left(V_{\xi^{\prime}}+\phi_{\xi^{\prime}}\right)\right|^{2}+\varepsilon^{2}\left(V_{\xi^{\prime}}+\phi_{\xi^{\prime}}\right)^{2}\right] d y & =\int_{\Omega_{p}} a(\varepsilon y)\left(V_{\xi^{\prime}}+\phi_{\xi^{\prime}}\right)^{p+1} d y+\sum_{i=1}^{m} \sum_{j=1}^{J_{i}} c_{i j}\left(\xi^{\prime}\right) \int_{\Omega_{p}} \chi_{i} Z_{i j} V_{\xi^{\prime}} d y \\
& =\int_{\Omega_{p}} a(\varepsilon y)\left(V_{\xi^{\prime}}+\phi_{\xi^{\prime}}\right)^{p+1} d y+O\left(\frac{1}{p^{4}}\right)
\end{aligned}
$$

because $V_{\xi^{\prime}}=O(1)$ and $\left|c_{i j}\left(\xi^{\prime}\right)\right|=O\left(p^{-4} \mu_{i}^{-1}\right)$. Hence, by (2.27), (4.2) and (5.3) we can write

$$
\begin{aligned}
F_{p}(\xi)= & \left(\frac{1}{2}-\frac{1}{p+1}\right) \varepsilon^{-4 /(p-1)} \int_{\Omega_{p}} a(\varepsilon y)\left[\left|\nabla\left(V_{\xi^{\prime}}+\phi_{\xi^{\prime}}\right)\right|^{2}+\varepsilon^{2}\left(V_{\xi^{\prime}}+\phi_{\xi^{\prime}}\right)^{2}\right] d y+O\left(\frac{1}{p^{5}}\right) \\
= & \left(\frac{1}{2}-\frac{1}{p+1}\right) \varepsilon^{-4 /(p-1)}\left\{\int_{\Omega_{p}} a(\varepsilon y)\left(\left|\nabla V_{\xi^{\prime}}\right|^{2}+\varepsilon^{2} V_{\xi^{\prime}}^{2}\right) d y\right. \\
& \left.+2 \int_{\Omega_{p}} a(\varepsilon y)\left(\nabla V_{\xi^{\prime}} \nabla \phi_{\xi^{\prime}}+\varepsilon^{2} V_{\xi^{\prime}} \phi_{\xi^{\prime}}\right) d y+\int_{\Omega_{p}} a(\varepsilon y)\left(\left|\nabla \phi_{\xi^{\prime}}\right|^{2}+\varepsilon^{2} \phi_{\xi^{\prime}}^{2}\right) d y\right\}+O\left(\frac{1}{p^{5}}\right) \\
= & \left(\frac{1}{2}-\frac{1}{p+1}\right) \int_{\Omega} a(x)\left(\left|\nabla U_{\xi}\right|^{2}+U_{\xi}^{2}\right) d x+O\left(\frac{1}{p^{3}}\left|\int_{\Omega} a(x)\left(\left|\nabla U_{\xi}\right|^{2}+U_{\xi}^{2}\right) d x\right|^{1 / 2}+\frac{1}{p^{5}}\right)
\end{aligned}
$$


uniformly for any points $\xi=\left(\xi_{1}, \ldots, \xi_{m}\right) \in \mathcal{O}_{p}$. Now, in view of $(2.9),(2.10),(2.16),(2.17),(2.18),(2.20)$ and $(2.21)$ we have

$$
\begin{aligned}
\int_{\Omega} a(x)\left(\left|\nabla U_{\xi}\right|^{2}+U_{\xi}^{2}\right) d x=\int_{\Omega} a(x)\left(-\Delta_{a} U_{\xi}+U_{\xi}\right) U_{\xi} d x \\
=\sum_{i=1}^{m} \frac{1}{\gamma \mu_{i}^{2 /(p-1)}} \int_{\Omega \cap B \frac{1}{p^{2 \kappa}}\left(\xi_{i}\right)} a(x)\left[e^{U_{\delta_{i}, \xi_{i}}}-\frac{1}{p \delta_{i}^{2}} \Delta \omega_{1}\left(\frac{x-\xi_{i}}{\delta_{i}}\right)-\frac{1}{p^{2} \delta_{i}^{2}} \Delta \omega_{2}\left(\frac{x-\xi_{i}}{\delta_{i}}\right)\right] U_{\xi} d x+o\left(\delta_{i}\right) \\
=\sum_{i=1}^{m} \frac{1}{\gamma^{2} \mu_{i}^{4 /(p-1)}} \int_{\left(\frac{1}{\delta_{i}}\left(\Omega-\left\{\xi_{i}\right\}\right)\right) \cap B \frac{1}{\delta_{i} p^{2 \kappa}}(0)} a\left(\xi_{i}+\delta_{i} z\right)\left[\frac{8}{\left(1+|z|^{2}\right)^{2}}-\frac{1}{p} \Delta \omega_{1}(z)-\frac{1}{p^{2}} \Delta \omega_{2}(z)\right] \\
\quad \times\left[p+U_{1,0}(z)+\frac{1}{p} \omega_{1}(z)+\frac{1}{p^{2}} \omega_{2}(z)+O\left(\delta_{i}^{\alpha}|z|^{\alpha}+\sum_{k=1}^{m} \delta_{k}^{\alpha / 2}\right)\right] d z+o\left(\delta_{i}\right) \\
=\sum_{i=1}^{m} \frac{c_{i} a\left(\xi_{i}\right)}{\gamma^{2} \mu_{i}^{4 /(p-1)}}\left[p+\mathcal{K}+O\left(\frac{1}{p}\right)\right]+o\left(\delta_{i}\right) .
\end{aligned}
$$

Recalling that $\gamma=p^{p /(p-1)} e^{-p /(2 p-2)}$, we get

$$
\frac{1}{\gamma^{2}}=\frac{e}{p^{2}}\left[1-\frac{2 \log p}{p}+\frac{1}{p}+O\left(\frac{\log ^{2} p}{p^{2}}\right)\right], \quad \frac{1}{\mu_{i}^{4 /(p-1)}}=1-\frac{4 \log \mu_{i}}{p}+O\left(\frac{\log ^{2} \mu_{i}}{p^{2}}\right),
$$

and then, by $(2.23)$,

$$
\frac{1}{\gamma^{2} \mu_{i}^{4 /(p-1)}}=\frac{e}{p^{2}}\left[1-\frac{2 \log p}{p}-\frac{4 \log \mu_{i}}{p}+\frac{1}{p}+O\left(\frac{\log ^{2} p}{p^{2}}\right)\right] .
$$

Therefore,

$$
F_{p}(\xi)=\frac{e}{2 p} \sum_{i=1}^{m} c_{i} a\left(\xi_{i}\right)\left\{1-\frac{2 \log p}{p}-\frac{4 \log \mu_{i}}{p}+\frac{\mathcal{K}-1}{p}\right\}+O\left(\frac{\log ^{2} p}{p^{3}}\right),
$$

which, together with the expansion of $\mu_{i}$ in (2.25), easily implies that (6.1) holds.

\section{Proofs of Theorems}

Proof of Theorem 1.1. According to Proposition 5.1, $U_{\xi}+\widetilde{\phi}_{\xi}$ is a solution of problem (1.5) if we adjust $\xi=$ $\left(\xi_{1}, \ldots, \xi_{m}\right) \in \mathcal{O}_{p}$ so that it is a critical point of $F_{p}$ defined in (5.2). For this aim, we only choose points $\xi=$ $\left(\xi_{1}, \ldots, \xi_{m}\right) \in \mathcal{O}_{p}$ in the following form of the parametrization

$$
\xi_{i} \equiv \xi_{i}(\mathbf{s}, \mathbf{t})=s_{i}-\frac{t_{i}}{p} \nu\left(s_{i}\right), \quad i=1, \ldots, l, \quad \text { but } \quad \xi_{i} \equiv \xi_{i}(\mathbf{s}, \mathbf{t})=s_{i}, \quad i=l+1, \ldots, m,
$$

where $\mathbf{s}=\left(s_{1}, \ldots, s_{m}\right) \in(\partial \Omega)^{m}$ and $\mathbf{t}=\left(t_{1}, \ldots, t_{l}\right) \in \mathbb{R}_{+}^{l}$ lie in the configuration space

$$
\Lambda_{d}=\left\{(\mathbf{s}, \mathbf{t}) \in(\partial \Omega)^{m} \times \mathbb{R}_{+}^{l}|| s_{i}-s_{k} \mid>2 d, \quad d<t_{\tilde{i}}<1 / d, \quad \forall i, k=1, \ldots, m, \tilde{i}=1, \ldots, l, i \neq k\right\},
$$

for any $d>0$ sufficiently small, fixed and independent of $p$. Thus we can easily prove that if $(\mathbf{s}, \mathbf{t})$ is a critical point of the reduced energy $\widehat{F}_{p}(\mathbf{s}, \mathbf{t}):=F_{p}(\xi(\mathbf{s}, \mathbf{t}))$ in $\Lambda_{d}$, then the function $U_{\xi(\mathbf{s}, \mathbf{t})}+\widetilde{\phi}_{\xi(\mathbf{s}, \mathbf{t})}$ is a solution of problem (1.5) with the qualitative properties predicted by Theorem 1.1. Therefore, we need first to compute the expansion of the reduced energy $\widehat{F}_{p}$ with the aid of Lemmas 2.1-2.2, Corollary 2.3 and Proposition 6.1.

Using the smooth property of $a(x)$ over $\bar{\Omega}$, we can perform a Taylor expansion around each boundary point $s_{i}$ along the inner normal vector $-\nu\left(s_{i}\right)$ to derive that

$$
a\left(\xi_{i}\right)=a\left(s_{i}\right)-\frac{t_{i}}{p} \partial_{\nu} a\left(s_{i}\right)+O\left(\frac{t_{i}^{2}}{p^{2}}\right), \quad \forall i=1, \ldots, l .
$$


From the expansions of the Robin's function in (2.4)-(2.5) and the regularity of the vector function $T(x)$, we obtain

$$
H\left(\xi_{i}, \xi_{i}\right)=-\frac{1}{2 \pi} \log \left(\frac{2 t_{i}}{p}\right)+\tilde{z}\left(s_{i}, s_{i}\right)+O\left(\frac{t_{i}^{\alpha}}{p^{\alpha}}\right), \quad \forall i=1, \ldots, l .
$$

On the other hand, from the expansions of the regular part of Green's function in (2.2)-(2.3), we find that if $i, k=1, \ldots, l$ with $i \neq k$,

$$
\begin{aligned}
G\left(\xi_{i}, \xi_{k}\right)= & -\frac{1}{2 \pi} \log \left|s_{i}-s_{k}-\frac{t_{i}}{p} \nu\left(s_{i}\right)+\frac{t_{k}}{p} \nu\left(s_{k}\right)\right|-\frac{1}{2 \pi} \log \left|s_{i}-s_{k}-\frac{t_{i}}{p} \nu\left(s_{i}\right)-\frac{t_{k}}{p} \nu\left(s_{k}\right)\right| \\
& +\frac{t_{k}}{\pi p}\left\langle\nabla \log a\left(s_{k}\right), \nabla T\left(s_{i}-s_{k}\right) \cdot \nu\left(s_{k}\right)\right\rangle-\frac{t_{k}}{\pi p}\left\langle(\nabla \times(\nabla \log a))\left(s_{k}\right) \cdot \nu\left(s_{k}\right), T\left(s_{i}-s_{k}\right)\right\rangle \\
& +\tilde{\mathrm{z}}\left(s_{i}, s_{k}\right)-\frac{1}{p}\left\langle\nabla_{\left(s_{i}, s_{k}\right)} \tilde{\mathrm{z}}\left(s_{i}, s_{k}\right),\left(t_{i} \nu\left(s_{i}\right), t_{k} \nu\left(s_{k}\right)\right)\right\rangle+O\left(\frac{t_{i}^{2}+t_{k}^{2}}{p^{2}}\right),
\end{aligned}
$$

while if $i=l+1, \ldots, m$ and $k=1, \ldots, l$,

$$
\begin{aligned}
G\left(\xi_{i}, \xi_{k}\right)= & -\frac{1}{2 \pi} \log \left|s_{i}-s_{k}+\frac{t_{k}}{p} \nu\left(s_{k}\right)\right|-\frac{1}{2 \pi} \log \left|s_{i}-s_{k}-\frac{t_{k}}{p} \nu\left(s_{k}\right)\right| \\
& +\frac{t_{k}}{\pi p}\left\langle\nabla \log a\left(s_{k}\right), \nabla T\left(s_{i}-s_{k}\right) \cdot \nu\left(s_{k}\right)\right\rangle-\frac{t_{k}}{\pi p}\left\langle(\nabla \times(\nabla \log a))\left(s_{k}\right) \cdot \nu\left(s_{k}\right), T\left(s_{i}-s_{k}\right)\right\rangle \\
& +\tilde{z}\left(s_{i}, s_{k}\right)-\frac{t_{k}}{p}\left\langle\nabla_{s_{k}} \tilde{z}\left(s_{i}, s_{k}\right), \nu\left(s_{k}\right)\right\rangle+O\left(\frac{t_{k}^{2}}{p^{2}}\right) .
\end{aligned}
$$

Substituting (2.18), (7.2)-(7.5) into (6.1) and using the fact that $a\left(\xi_{i}\right) G\left(\xi_{i}, \xi_{k}\right)=a\left(\xi_{k}\right) G\left(\xi_{k}, \xi_{i}\right)$ for all $i, k=1, \ldots, m$ with $i \neq k$, we conclude that $\widehat{F}_{p}$ becomes

$$
\widehat{F}_{p}(\mathbf{s}, \mathbf{t})=\frac{e}{2 p}\left\{8 \pi \sum_{i=1}^{l}\left[a\left(s_{i}\right)+\frac{1}{p}\left(4 a\left(s_{i}\right) \log t_{i}-t_{i} \partial_{\nu} a\left(s_{i}\right)\right)\right]+4 \pi \sum_{k=l+1}^{m} a\left(s_{k}\right)+\Gamma_{p}(\mathbf{s})\right\}+O\left(\frac{|\mathbf{t}|^{\alpha}}{p^{2+\alpha}}+\frac{\log ^{2} p}{p^{3}}\right)
$$

$C^{1}$-uniformly in $\Lambda_{d}$, where $\Gamma_{p}(\mathbf{s})$ is a smooth function of $\mathbf{s}$ satisfying that $\Gamma_{p}(\mathbf{s})$ and $\left|\nabla \Gamma_{p}(\mathbf{s})\right|$ uniformly converge to zero as $p \rightarrow+\infty$.

We seek a critical point of $\widehat{F}_{p}$ in $\Lambda_{d}$. Let $\partial_{T\left(s_{i}\right)}$ be the tangential derivative which is defined on $s_{i} \in \partial \Omega$. Set

$$
A\left(s_{i}, t_{i}\right)=a\left(s_{i}\right)+\frac{1}{p}\left(4 a\left(s_{i}\right) \log t_{i}-t_{i} \partial_{\nu} a\left(s_{i}\right)\right), \quad i=1, \ldots, l .
$$

Then

$$
\partial_{T\left(s_{i}\right)} A\left(s_{i}, t_{i}\right)=\left(1+\frac{4 \log t_{i}}{p}\right) \partial_{T} a\left(s_{i}\right)-\frac{t_{i}}{p} \partial_{T} \partial_{\nu} a\left(s_{i}\right), \quad \partial_{t_{i}} A\left(s_{i}, t_{i}\right)=\frac{1}{p}\left(\frac{4}{t_{i}} a\left(s_{i}\right)-\partial_{\nu} a\left(s_{i}\right)\right) .
$$

In view of $\partial_{\nu} a\left(\xi_{i}^{*}\right)>0$ with $i=1, \ldots, l$, we have that for any sufficiently small $d>0$ and for any $s_{i} \in B_{d}\left(\xi_{i}^{*}\right) \cap \partial \Omega$, there exists a unique positive $t_{i}=t_{i}\left(s_{i}\right)=4 a\left(s_{i}\right) / \partial_{\nu} a\left(s_{i}\right)$ such that $\partial_{t_{i}} A\left(s_{i}, t_{i}\right)=0$ and $\partial_{t_{i} t_{i}}^{2} A\left(s_{i}, t_{i}\right)<0$. Set $t_{i}^{*}=t_{i}\left(\xi_{i}^{*}\right)$, $i=1, \ldots, l$. Since $\xi_{1}^{*}, \ldots, \xi_{m}^{*}$ are $m$ different strict local maximum or strict local minimum points of $a(x)$ on $\partial \Omega$, we find that for any $i=1, \ldots, l$, any sufficiently small $d>0$ and any sufficiently large $p>1$, the Brouwer degrees $\operatorname{deg}\left(\left(\partial_{T\left(s_{i}\right)} A, \partial_{t_{i}} A\right),\left(B_{d}\left(\xi_{i}^{*}\right) \cap \partial \Omega\right) \times\left(t_{i}^{*}-d, t_{i}^{*}+d\right), 0\right)$ and $\operatorname{deg}\left(\left(\partial_{T\left(s_{l+1}\right)} a, \ldots, \partial_{T\left(s_{m}\right)} a\right), \prod_{k=l+1}^{m}\left(B_{d}\left(\xi_{k}^{*}\right) \cap \partial \Omega\right), 0\right)$ are well defined (see $[7,37])$. By the definition and homotopy invariance of topological degree we deduce

$$
\begin{aligned}
\operatorname{deg}\left(\left(\partial_{T\left(s_{i}\right)} A, \partial_{t_{i}} A\right),\left(B_{d}\left(\xi_{i}^{*}\right) \cap \partial \Omega\right) \times\left(t_{i}^{*}-d, t_{i}^{*}+d\right), 0\right) & \\
= & \operatorname{deg}\left(\left(\partial_{T} a\left(s_{i}\right), \partial_{t_{i}} A\right),\left(B_{d}\left(\xi_{i}^{*}\right) \cap \partial \Omega\right) \times\left(t_{i}^{*}-d, t_{i}^{*}+d\right), 0\right) \\
= & \operatorname{sign} \operatorname{det}\left[\left(\begin{array}{cc}
\partial_{T T}^{2} a\left(\xi_{i}^{*}\right) & \frac{1}{p}\left(\frac{4}{t_{i}^{*}} \partial_{T} a\left(\xi_{i}^{*}\right)-\partial_{T} \partial_{\nu} a\left(\xi_{i}^{*}\right)\right) \\
0 & -\frac{1}{p} \frac{4}{\left(t_{i}^{*}\right)^{2}} a\left(\xi_{i}^{*}\right)
\end{array}\right)\right]= \pm 1 \neq 0,
\end{aligned}
$$


and

$$
\operatorname{deg}\left(\left(\partial_{T\left(s_{l+1}\right)} a, \ldots, \partial_{T\left(s_{m}\right)} a\right), \prod_{k=l+1}^{m}\left(B_{d}\left(\xi_{k}^{*}\right) \cap \partial \Omega\right), 0\right)=\operatorname{sign}\left(\prod_{k=l+1}^{m} \partial_{T T}^{2} a\left(\xi_{k}^{*}\right)\right)= \pm 1 \neq 0 .
$$

Furthermore, using the properties of Brouwer degree, by (7.6) we conclude

$$
\begin{aligned}
& \operatorname{deg}\left(\nabla_{(T(\mathbf{s}), \mathbf{t})} \widehat{F}_{p}(\mathbf{s}, \mathbf{t}), \prod_{i=1}^{l}\left(B_{d}\left(\xi_{i}^{*}\right) \cap \partial \Omega\right) \times \prod_{k=l+1}^{m}\left(B_{d}\left(\xi_{k}^{*}\right) \cap \partial \Omega\right) \times \prod_{i=1}^{l}\left(t_{i}^{*}-d, t_{i}^{*}+d\right), 0\right) \\
&= \operatorname{deg}\left(\left(\partial_{T\left(s_{1}\right)} A, \ldots, \partial_{T\left(s_{l}\right)} A, \partial_{T\left(s_{l+1}\right)} a, \ldots, \partial_{T\left(s_{m}\right)} a, \partial_{t_{1}} A, \ldots, \partial_{t_{l}} A\right),\right. \\
&\left.\prod_{i=1}^{l}\left(B_{d}\left(\xi_{i}^{*}\right) \cap \partial \Omega\right) \times \prod_{k=l+1}^{m}\left(B_{d}\left(\xi_{k}^{*}\right) \cap \partial \Omega\right) \times \prod_{i=1}^{l}\left(t_{i}^{*}-d, t_{i}^{*}+d\right), 0\right) \\
&=\prod_{i=1}^{l} \operatorname{deg}\left(\left(\partial_{T\left(s_{i}\right)} A, \partial_{t_{i}} A\right),\left(B_{d}\left(\xi_{i}^{*}\right) \cap \partial \Omega\right) \times\left(t_{i}^{*}-d, t_{i}^{*}+d\right), 0\right) \\
& \quad \times \operatorname{deg}\left(\left(\partial_{T\left(s_{l+1}\right)} a, \ldots, \partial_{T\left(s_{m}\right)} a\right), \prod_{k=l+1}^{m}\left(B_{d}\left(\xi_{k}^{*}\right) \cap \partial \Omega\right), 0\right)
\end{aligned}
$$

$\neq 0$.

This implies that for any $p>1$ large enough, there exists $\left(\mathbf{s}^{p}, \mathbf{t}^{p}\right) \in \Lambda_{d}$ such that $\nabla_{(T(\mathbf{s}), \mathbf{t})} \widehat{F}_{p}\left(\mathbf{s}^{p}, \mathbf{t}^{p}\right)=0$. In particular, $\mathbf{s}^{p}=\left(s_{1}^{p}, \ldots, s_{m}^{p}\right) \rightarrow\left(\xi_{1}^{*}, \ldots, \xi_{m}^{*}\right)$ as $p \rightarrow+\infty$, which completes the proof.

Proof of Theorem 1.2. According to Proposition 5.1, we need to find a critical point $\xi^{p}=\left(\xi_{1}^{p}, \ldots, \xi_{m}^{p}\right) \in$ $\Omega^{l} \times(\partial \Omega)^{m-l}$ of $F_{p}$ such that points $\xi_{1}^{p}, \ldots, \xi_{m}^{p}$ accumulate to $\xi_{*}$. Using (1.7), (2.18), (6.1), Lemma 2.1 and the fact that $a\left(\xi_{i}\right) G\left(\xi_{i}, \xi_{k}\right)=a\left(\xi_{k}\right) G\left(\xi_{k}, \xi_{i}\right)$ for all $i, k=1, \ldots, m$ with $i \neq k$, we conclude that $F_{p}$ becomes

$$
\begin{gathered}
F_{p}(\xi)=\frac{e}{2 p^{2}}\left\{8 \pi \sum_{i=1}^{l} a\left(\xi_{i}\right)\left[p-2 \log p-8 \pi H\left(\xi_{i}, \xi_{i}\right)-8 \pi \sum_{k=1, k \neq i}^{l} G\left(\xi_{i}, \xi_{k}\right)\right]-64 \pi^{2} \sum_{i=1}^{l} \sum_{k=l+1}^{m} a\left(\xi_{k}\right) G\left(\xi_{k}, \xi_{i}\right)\right. \\
\left.+4 \pi \sum_{i=l+1}^{m} a\left(\xi_{i}\right)\left[p-2 \log p+4 \sum_{k=l+1, k \neq i}^{m} \log \left|\xi_{i}-\xi_{k}\right|\right]+O(1)\right\}
\end{gathered}
$$

$C^{0}$-uniformly in $\mathcal{O}_{d, p}^{*}:=\left\{\xi=\left(\xi_{1}, \ldots, \xi_{m}\right) \in\left(B_{d}\left(\xi_{*}\right) \cap \Omega\right)^{l} \times\left(B_{d}\left(\xi_{*}\right) \cap \partial \Omega\right)^{m-l}|\underset{i, k=1, \ldots, m, i \neq k}{\min }| \xi_{i}-\xi_{k} \mid>\frac{1}{p^{\kappa}}\right.$, $\left.\min _{1 \leq i \leq l} \operatorname{dist}\left(\xi_{i}, \partial \Omega\right)>\frac{1}{p^{\kappa}}\right\}$ for any $d>0$ sufficiently small, fixed and independent of $p$. Here we claim that for any $p>1$ large enough, the following maximization problem

$$
\max _{\left(\xi_{1}, \ldots, \xi_{m}\right) \in \overline{\mathcal{O}}_{d, p}^{*}} F_{p}\left(\xi_{1}, \ldots, \xi_{m}\right)
$$

has a solution in the interior of $\mathcal{O}_{d, p}^{*}$. Once this claim is proven, we can easily get the qualitative properties of solutions of (1.5) as predicted in Theorem 1.3.

Let $\xi^{p}=\left(\xi_{1}^{p}, \ldots, \xi_{m}^{p}\right)$ be the maximizer of $F_{p}$ over $\overline{\mathcal{O}}_{d, p}^{*}$. We are led to prove that $\xi^{p}$ lies in the interior of $\mathcal{O}_{d, p}^{*}$. First, we obtain a lower bound for $F_{p}$ over $\overline{\mathcal{O}}_{d, p}^{*}$. Let us consider a smooth change of variables

$$
H_{\xi_{*}}^{p}(y)=e^{p / 2} H_{\xi_{*}}\left(e^{-p / 2} y\right),
$$


where $H_{\xi_{*}}: B_{d}\left(\xi_{*}\right) \mapsto \mathcal{M}$ is a diffeomorphism and $\mathcal{M}$ is an open neighborhood of the origin such that $H_{\xi_{*}}\left(B_{d}\left(\xi_{*}\right) \cap\right.$ $\Omega)=\mathcal{M} \cap \mathbb{R}_{+}^{2}$ and $H_{\xi_{*}}\left(B_{d}\left(\xi_{*}\right) \cap \partial \Omega\right)=\mathcal{M} \cap \partial \mathbb{R}_{+}^{2}$. Let

$$
\xi_{i}^{0}=\xi_{*}-\frac{t_{i}}{\sqrt{p}} \nu\left(\xi_{*}\right), \quad i=1, \ldots, l, \quad \text { but } \quad \xi_{i}^{0}=e^{-p / 2}\left(H_{\xi_{*}}^{p}\right)^{-1}\left(\frac{e^{p / 2}}{\sqrt{p}} \hat{\xi}_{i}^{0}\right), \quad i=l+1, \ldots, m,
$$

where $t_{i}>0$ and $\hat{\xi}_{i}^{0} \in \mathcal{M} \cap \partial \mathbb{R}_{+}^{2}$ satisfy $t_{i+1}-t_{i}=\rho,\left|\hat{\xi}_{i}^{0}-\hat{\xi}_{i+1}^{0}\right|=\rho$ for all $\rho>0$ sufficiently small, fixed and independent of $p$. From the expansion $\left(H_{\xi_{*}}^{p}\right)^{-1}(z)=e^{p / 2} \xi_{*}+z+O\left(e^{-p / 2}|z|\right)$, we get

$$
\xi_{i}^{0}=\xi_{*}+\frac{1}{\sqrt{p}} \hat{\xi}_{i}^{0}+O\left(\frac{e^{-p / 2}}{\sqrt{p}}\left|\hat{\xi}_{i}^{0}\right|\right), \quad i=l+1, \ldots, m .
$$

As $\kappa>1$, we find $\xi^{0}=\left(\xi_{1}^{0}, \ldots, \xi_{m}^{0}\right) \in \mathcal{O}_{d, p}^{*}$. Since $\xi_{*} \in \partial \Omega$ is a strict local maximum point of $a(x)$ over $\bar{\Omega}$ and satisfies $\partial_{\nu} a\left(\xi_{*}\right)=\left\langle\nabla a\left(\xi_{*}\right), \nu\left(\xi_{*}\right)\right\rangle=0$, there exists a constant $C>0$ independent of $p$ such that

$$
a\left(\xi_{*}\right)-\frac{C}{p} \leq a\left(\xi_{i}^{0}\right)<a\left(\xi_{*}\right), \quad i=1, \ldots, m .
$$

On the other hand, from definition (1.7), Lemma 2.2 and Corollary 2.3, we can compute that for any $i=1, \ldots, l$ and $k=1, \ldots, m$ with $i \neq k$,

$$
H\left(\xi_{i}^{0}, \xi_{i}^{0}\right)=\frac{1}{4 \pi} \log p+O(1), \quad G\left(\xi_{k}^{0}, \xi_{i}^{0}\right)=H\left(\xi_{k}^{0}, \xi_{i}^{0}\right)-\frac{1}{2 \pi} \log \left|\xi_{k}^{0}-\xi_{i}^{0}\right|=\frac{1}{2 \pi} \log p+O(1) .
$$

Additionally, for any $i, k=l+1, \ldots, m$ with $i \neq k$,

$$
\log \left|\xi_{i}^{0}-\xi_{k}^{0}\right|=-\frac{1}{2} \log p+O(1) .
$$

Hence by (7.7), we find

$$
\max _{\xi \in \overline{\mathcal{O}}_{d, p}^{*}} F_{p}(\xi) \geq F_{p}\left(\xi^{0}\right) \geq \frac{e}{2 p^{2}}\left\{4 \pi(m+l) p a\left(\xi_{*}\right)-8 \pi(m+l)^{2} a\left(\xi_{*}\right) \log p+O(1)\right\} .
$$

Next, we suppose $\xi^{p}=\left(\xi_{1}^{p}, \ldots, \xi_{m}^{p}\right) \in \partial \mathcal{O}_{d, p}^{*}$. Then there exist four cases:

C1. There exists an $i_{0} \in\{1, \ldots, l\}$ such that $\xi_{i_{0}}^{p} \in \partial B_{d}\left(\xi_{*}\right) \cap \Omega$, in which case, $a\left(\xi_{i_{0}}^{p}\right)<a\left(\xi_{*}\right)-d_{0}$ for some $d_{0}>0$ independent of $p$;

C2. There exists an $i_{0} \in\{l+1, \ldots, m\}$ such that $\xi_{i_{0}}^{p} \in \partial B_{d}\left(\xi_{*}\right) \cap \partial \Omega$, in which case, $a\left(\xi_{i_{0}}^{p}\right)<a\left(\xi_{*}\right)-d_{0}$ for some $d_{0}>0$ independent of $p$

C3. There exists an $i_{0} \in\{1, \ldots, l\}$ such that $\operatorname{dist}\left(\xi_{i_{0}}^{p}, \partial \Omega\right)=p^{-\kappa}$;

C4. There exist indices $i_{0}, k_{0}, i_{0} \neq k_{0}$ such that $\left|\xi_{i_{0}}^{p}-\xi_{k_{0}}^{p}\right|=p^{-\kappa}$.

Observe that for all $i=1, \ldots, l$ and $k=1, \ldots, m$ with $i \neq k$, by (1.6), (2.2), (2.4) and the maximum principle,

$$
G\left(\xi_{k}^{p}, \xi_{i}^{p}\right)>0, \quad H\left(\xi_{k}^{p}, \xi_{i}^{p}\right)>0 \quad \text { and } \quad H\left(\xi_{i}^{p}, \xi_{i}^{p}\right)=-\frac{1}{2 \pi} \log \left[\operatorname{dist}\left(\xi_{i}^{p}, \partial \Omega\right)\right]+O(1) .
$$

In the first and second cases, by (7.7) and (7.9) we get

$$
\max _{\xi \in \overline{\mathcal{O}}_{d, p}^{*}} F_{p}(\xi)=F_{p}\left(\xi^{p}\right) \leq \frac{e}{2 p^{2}}\left\{4 \pi p\left[(m+l) a\left(\xi_{*}\right)-d_{0}\right]+O(\log p)\right\},
$$

which contradicts to (7.8). This shows that $a\left(\xi_{i}^{p}\right) \rightarrow a\left(\xi_{*}\right)$. Using the assumption of $a(x)$ over $\bar{\Omega}$, we deduce $\xi_{i}^{p} \rightarrow \xi_{*}$ for all $i=1, \ldots, m$.

In the third case, by (7.7) and (7.9) we get

$$
\begin{aligned}
\max _{\xi \in \overline{\mathcal{O}}_{d, p}^{*}} F_{p}(\xi)=F_{p}\left(\xi^{p}\right) & \leq \frac{e}{2 p^{2}}\left\{4 \pi(m+l)(p-2 \log p) a\left(\xi_{*}\right)-64 \pi^{2} a\left(\xi_{i_{0}}^{p}\right) H\left(\xi_{i_{0}}^{p}, \xi_{i_{0}}^{p}\right)+O(1)\right\} \\
& \leq \frac{e}{2 p^{2}}\left\{4 \pi(m+l)(p-2 \log p) a\left(\xi_{*}\right)-32 \pi \kappa a\left(\xi_{i_{0}}^{p}\right) \log p+O(1)\right\} .
\end{aligned}
$$


In the last case, by (7.7) and (7.9) we get, if $i_{0} \in\{1, \ldots, m\}$ and $k_{0} \in\{1, \ldots, l\}$,

$$
\begin{aligned}
\max _{\xi \in \overline{\mathcal{O}}_{d, p}^{*}} F_{p}(\xi)=F_{p}\left(\xi^{p}\right) & \leq \frac{e}{2 p^{2}}\left\{4 \pi(m+l)(p-2 \log p) a\left(\xi_{*}\right)+32 \pi a\left(\xi_{i_{0}}^{p}\right) \log \left|\xi_{i_{0}}^{p}-\xi_{k_{0}}^{p}\right|+O(1)\right\} \\
& \leq \frac{e}{2 p^{2}}\left\{4 \pi(m+l)(p-2 \log p) a\left(\xi_{*}\right)-32 \pi \kappa a\left(\xi_{i_{0}}^{p}\right) \log p+O(1)\right\},
\end{aligned}
$$

while if $i_{0} \in\{l+1, \ldots, m\}$ and $k_{0} \in\{l+1, \ldots, m\}$,

$$
\begin{aligned}
\max _{\xi \in \overline{\mathcal{O}}_{d, p}^{*}} F_{p}(\xi)=F_{p}\left(\xi^{p}\right) & \leq \frac{e}{2 p^{2}}\left\{4 \pi(m+l)(p-2 \log p) a\left(\xi_{*}\right)+16 \pi a\left(\xi_{i_{0}}^{p}\right) \log \left|\xi_{i_{0}}^{p}-\xi_{k_{0}}^{p}\right|+O(1)\right\} \\
& \leq \frac{e}{2 p^{2}}\left\{4 \pi(m+l)(p-2 \log p) a\left(\xi_{*}\right)-16 \pi \kappa a\left(\xi_{i_{0}}^{p}\right) \log p+O(1)\right\} .
\end{aligned}
$$

Comparing (7.11)-(7.13) with (7.8), we obtain

$$
8 \pi(m+l) a\left(\xi_{*}\right) \log p+32 \pi \kappa a\left(\xi_{i_{0}}^{p}\right) \log p+O(1) \leq 8 \pi(m+l)^{2} a\left(\xi_{*}\right) \log p+O(1),
$$

which is impossible by the choice of $\kappa$ in (2.8).

\section{ACKNOWLEDGMENTS}

The author warmly thanks the anonymous referee for his or her nice and valuable comments on this manuscript. This research is supported by the Fundamental Research Funds for the Central Universities under Grant No. KYZ201649, and the National Natural Science Foundation of China under Grant Nos. 11601232, 11671354 and 11775116 .

\section{Appendix}

Proof of lemma 2.4. Observe that for any $\beta \in(0,1)$, by (2.6), (2.10) and (2.13),

$$
\left\{\begin{aligned}
-\Delta_{a} H_{i}+H_{i}= & \frac{1}{\gamma \mu_{i}^{2 /(p-1)}}\left\{\left(-4+\frac{C_{1}}{p}+\frac{C_{2}}{p^{2}}\right)\left[\frac{\left(x-\xi_{i}\right) \cdot \nabla \log a(x)}{\delta_{i}^{2}+\left|x-\xi_{i}\right|^{2}}-\frac{1}{2} \log \left(\delta_{i}^{2}+\left|x-\xi_{i}\right|^{2}\right)\right]-\log \left(8 \delta_{i}^{2}\right)\right. \\
& +\left(\frac{C_{1}}{p}+\frac{C_{2}}{p^{2}}\right) \log \delta_{i}+\frac{1}{p} O_{L^{\infty}\left(\Omega \backslash B_{\delta_{i}^{\beta / 2}}\left(\xi_{i}\right)\right)}\left(\frac{\delta_{i}}{\delta_{i}+\left|x-\xi_{i}\right|}+\frac{\delta_{i}}{\delta_{i}^{2}+\left|x-\xi_{i}\right|^{2}}\right) \\
& \left.+\frac{1}{p} O_{L^{\infty}\left(\Omega \cap B_{\delta_{i}^{\beta / 2}}\left(\xi_{i}\right)\right.}\left(\frac{\left|\left(x-\xi_{i}\right) \cdot \nabla \log a(x)\right|}{\delta_{i}^{2}+\left|x-\xi_{i}\right|^{2}}+\log \frac{\delta_{i}^{2}+\left|x-\xi_{i}\right|^{2}}{\delta_{i}^{2}}\right)\right\} \quad \text { in } \quad \Omega, \\
\frac{\partial H_{i}}{\partial \nu}=-\frac{1}{\gamma \mu_{i}^{2 /(p-1)}}\left\{\left(-4+\frac{C_{1}}{p}+\frac{C_{2}}{p^{2}}\right) \frac{\left(x-\xi_{i}\right) \cdot \nu(x)}{\delta_{i}^{2}+\left|x-\xi_{i}\right|^{2}}+\frac{1}{p} O_{L^{\infty}\left(\partial \Omega \backslash B_{\delta_{i}^{\beta / 2}}\left(\xi_{i}\right)\right.}\left(\frac{\delta_{i}}{\delta_{i}^{2}+\left|x-\xi_{i}\right|^{2}}\right)\right. & \text { on } \partial \Omega .
\end{aligned}\right.
$$

From (1.6)-(1.7) we have that the regular part of Green's function, $H\left(x, \xi_{i}\right)$, satisfies

$$
\begin{cases}-\Delta_{a} H\left(x, \xi_{i}\right)+H\left(x, \xi_{i}\right)=\frac{4}{c_{i}} \log \left|x-\xi_{i}\right|-\frac{4}{c_{i}} \frac{\left(x-\xi_{i}\right) \cdot \nabla \log a(x)}{\left|x-\xi_{i}\right|^{2}} & \text { in } \Omega, \\ \frac{\partial H\left(x, \xi_{i}\right)}{\partial \nu}=\frac{4}{c_{i}} \frac{\left(x-\xi_{i}\right) \cdot \nu(x)}{\left|x-\xi_{i}\right|^{2}} & \text { on } \partial \Omega .\end{cases}
$$


So, if we set

$$
Z_{i}(x)=\gamma \mu_{i}^{2 /(p-1)} H_{i}(x)-\left(4-\frac{C_{1}}{p}-\frac{C_{2}}{p^{2}}\right) \frac{c_{i}}{4} H\left(x, \xi_{i}\right)+\log \left(8 \delta_{i}^{2}\right)-\left(\frac{C_{1}}{p}+\frac{C_{2}}{p^{2}}\right) \log \delta_{i}
$$

then $Z_{i}(x)$ satisfies

$$
\left\{\begin{aligned}
-\Delta_{a} Z_{i}+Z_{i}= & \left(-4+\frac{C_{1}}{p}+\frac{C_{2}}{p^{2}}\right)\left[\frac{1}{2} \log \left(\frac{\left|x-\xi_{i}\right|^{2}}{\delta_{i}^{2}+\left|x-\xi_{i}\right|^{2}}\right)-\frac{\left(x-\xi_{i}\right) \cdot \nabla \log a(x)}{\left|x-\xi_{i}\right|^{2}} \cdot \frac{\delta_{i}^{2}}{\delta_{i}^{2}+\left|x-\xi_{i}\right|^{2}}\right] \\
& +\frac{1}{p} O_{L^{\infty}\left(\Omega \backslash B_{\delta_{i}^{\beta / 2}}\left(\xi_{i}\right)\right.}\left(\frac{\delta_{i}}{\delta_{i}+\left|x-\xi_{i}\right|}+\frac{\delta_{i}}{\delta_{i}^{2}+\left|x-\xi_{i}\right|^{2}}\right) \\
& +\frac{1}{p} O_{L^{\infty}}\left(\Omega \cap B_{\delta_{i}^{\beta / 2}}\left(\xi_{i}\right)\right)\left(\frac{\left|\left(x-\xi_{i}\right) \cdot \nabla \log a(x)\right|}{\delta_{i}^{2}+\left|x-\xi_{i}\right|^{2}}+\log \frac{\delta_{i}^{2}+\left|x-\xi_{i}\right|^{2}}{\delta_{i}^{2}}\right) \quad \text { in } \Omega, \\
\frac{\partial Z_{i}}{\partial \nu}= & \left(-4+\frac{C_{1}}{p}+\frac{C_{2}}{p^{2}}\right) \frac{\left(x-\xi_{i}\right) \cdot \nu(x)}{\left|x-\xi_{i}\right|^{2}} \cdot \frac{\delta_{i}^{2}}{\delta_{i}^{2}+\left|x-\xi_{i}\right|^{2}}+\frac{1}{p} O_{L^{\infty}\left(\partial \Omega \backslash B_{\delta_{i}^{\beta / 2}}\left(\xi_{i}\right)\right.}\left(\frac{\delta_{i}}{\delta_{i}^{2}+\left|x-\xi_{i}\right|^{2}}\right) \\
& +\frac{1}{p} O_{L^{\infty}\left(\partial \Omega \cap B_{\delta_{i}^{\beta / 2}}\left(\xi_{i}\right)\right.}\left(\frac{\left|\left(x-\xi_{i}\right) \cdot \nu(x)\right|}{\delta_{i}^{2}+\left|x-\xi_{i}\right|^{2}}\right)
\end{aligned}\right.
$$

Using polar coordinates with center $\xi_{i}$, i.e. $r=\left|x-\xi_{i}\right|$, and changing variables $s=r / \delta_{i}$, we estimate that for any $q>1$

$$
\begin{aligned}
\int_{\Omega}\left|\log \left(\frac{\left|x-\xi_{i}\right|^{2}}{\delta_{i}^{2}+\left|x-\xi_{i}\right|^{2}}\right)\right|^{q} d x & \leq 2 \pi \int_{0}^{\operatorname{diam}(\Omega)}\left|\log \left(\frac{r^{2}}{\delta_{i}^{2}+r^{2}}\right)\right|^{q} r d r \\
& \leq 2 \pi \delta_{i}^{2} \int_{0}^{+\infty}\left|\log \left(1+\frac{1}{s^{2}}\right)\right|^{q} s d s \\
& \leq C \delta_{i}^{2},
\end{aligned}
$$

and

$$
\begin{aligned}
\int_{\Omega \backslash B_{\delta_{i}^{\beta / 2}}\left(\xi_{i}\right)}\left|\frac{\delta_{i}}{\delta_{i}+\left|x-\xi_{i}\right|}+\frac{\delta_{i}}{\delta_{i}^{2}+\left|x-\xi_{i}\right|^{2}}\right|^{q} d x & \leq C \int_{\delta_{i}^{\beta / 2}}^{\operatorname{diam}(\Omega)}\left[\frac{\delta_{i}^{q}}{\left(\delta_{i}+r\right)^{q}}+\frac{\delta_{i}^{q}}{\left(\delta_{i}^{2}+r^{2}\right)^{q}}\right] r d r \\
& \leq C \int_{\delta_{i}^{\beta / 2-1}}^{+\infty}\left[\frac{\delta_{i}^{2}}{(1+s)^{q}}+\frac{\delta_{i}^{2-q}}{\left(1+s^{2}\right)^{q}}\right] s d s \\
& \leq C \delta_{i}^{\beta+q(1-\beta)}
\end{aligned}
$$

and for any $1<q<2$,

$$
\begin{aligned}
\int_{\Omega \cap B_{\delta_{i}^{\beta / 2}}\left(\xi_{i}\right)}\left|\frac{\left|\left(x-\xi_{i}\right) \cdot \nabla \log a(x)\right|}{\delta_{i}^{2}+\left|x-\xi_{i}\right|^{2}}+\log \frac{\delta_{i}^{2}+\left|x-\xi_{i}\right|^{2}}{\delta_{i}^{2}}\right|^{q} d x & \leq C \int_{0}^{\delta_{i}^{\beta / 2}}\left[\left(\frac{r}{\delta_{i}^{2}+r^{2}}\right)^{q}+\left|\log \left(\frac{\delta_{i}^{2}+r^{2}}{\delta_{i}^{2}}\right)\right|^{q}\right] r d r \\
& =C \delta_{i}^{2} \int_{0}^{\delta_{i}^{\beta / 2-1}}\left[\delta_{i}^{-q}\left(\frac{s}{1+s^{2}}\right)^{q}+\log ^{q}\left(1+s^{2}\right)\right] s d s \\
& \leq C \delta_{i}^{\beta(1-q / 2)},
\end{aligned}
$$


and

$$
\begin{aligned}
\int_{\Omega}\left|\frac{\left(x-\xi_{i}\right) \cdot \nabla \log a(x)}{\left|x-\xi_{i}\right|^{2}} \cdot \frac{\delta_{i}^{2}}{\delta_{i}^{2}+\left|x-\xi_{i}\right|^{2}}\right|^{q} d x & \leq C \int_{0}^{\operatorname{diam}(\Omega)}\left|\frac{\delta_{i}^{2}}{r\left(\delta_{i}^{2}+r^{2}\right)}\right|^{q} r d r \\
& \leq C \delta_{i}^{2-q} \int_{0}^{+\infty} \frac{s^{1-q}}{\left(1+s^{2}\right)^{q}} d s \\
& \leq C \delta_{i}^{2-q} .
\end{aligned}
$$

Thus for any $\xi_{i} \in \bar{\Omega}$ and any $1<q<2$,

$$
\left\|-\Delta_{a} Z_{i}+Z_{i}\right\|_{L^{q}(\Omega)} \leq C \delta_{i}^{\beta(1 / q-1 / 2)}
$$

As for the boundary terms, if $\xi_{i} \in \Omega$, by (2.7) we get, for any $x \in \partial \Omega$,

and further,

$$
\begin{gathered}
\left|\frac{\left(x-\xi_{i}\right) \cdot \nu(x)}{\left|x-\xi_{i}\right|^{2}} \cdot \frac{\delta_{i}^{2}}{\delta_{i}^{2}+\left|x-\xi_{i}\right|^{2}}\right| \leq \frac{\delta_{i}^{2}}{\left|x-\xi_{i}\right|^{3}} \leq \delta_{i}^{2} p^{3 \kappa}, \\
\frac{\delta_{i}}{\delta_{i}^{2}+\left|x-\xi_{i}\right|^{2}} \leq \frac{\delta_{i}}{\left|x-\xi_{i}\right|^{2}} \leq \delta_{i} p^{2 \kappa}
\end{gathered}
$$

$$
\left\|\frac{\partial Z_{i}}{\partial \nu}\right\|_{L^{\infty}(\partial \Omega)} \leq C \delta_{i} p^{2 \kappa-1}
$$

While if $\xi_{i} \in \partial \Omega$, using the fact that $\left|\left(x-\xi_{i}\right) \cdot \nu(x)\right| \leq C\left|x-\xi_{i}\right|^{2}$ for any $x \in \partial \Omega$ (see [5]), we estimate that for any $q>1$,

$$
\begin{gathered}
\int_{\partial \Omega}\left|\frac{\left(x-\xi_{i}\right) \cdot \nu(x)}{\left|x-\xi_{i}\right|^{2}} \cdot \frac{\delta_{i}^{2}}{\delta_{i}^{2}+\left|x-\xi_{i}\right|^{2}}\right|^{q} d x \leq C \int_{0}^{+\infty} \frac{\delta_{i}^{2 q}}{\left(\delta_{i}^{2}+r^{2}\right)^{q}} d r=C \delta_{i} \int_{0}^{+\infty} \frac{1}{\left(1+s^{2}\right)^{q}} d s \leq C \delta_{i}, \\
\int_{\partial \Omega \cap B_{\delta_{i}^{\beta / 2}}\left(\xi_{i}\right)}\left|\frac{\left(x-\xi_{i}\right) \cdot \nu(x)}{\delta_{i}^{2}+\left|x-\xi_{i}\right|^{2}}\right|^{q} d x \leq C \int_{\partial \Omega \cap B_{\delta_{i}^{\beta / 2}}\left(\xi_{i}\right)} \frac{\left|x-\xi_{i}\right|^{2 q}}{\left(\delta_{i}^{2}+\left|x-\xi_{i}\right|^{2}\right)^{q}} d x \leq C\left|\partial \Omega \cap B_{\delta_{i}^{\beta / 2}}\left(\xi_{i}\right)\right| \leq C \delta_{i}^{\beta / 2},
\end{gathered}
$$

and

$$
\int_{\partial \Omega \backslash B_{\delta_{i}^{\beta / 2}}\left(\xi_{i}\right)}\left|\frac{\delta_{i}}{\delta_{i}^{2}+\left|x-\xi_{i}\right|^{2}}\right|^{q} d x \leq C \int_{\delta_{i}^{\beta / 2}}^{|\partial \Omega|} \frac{\delta_{i}^{q}}{\left(\delta_{i}^{2}+r^{2}\right)^{q}} d r \leq C \int_{\delta_{i}^{\beta / 2}}^{|\partial \Omega|} \frac{\delta_{i}^{q}}{r^{2 q}} d r \leq C\left(\delta_{i}^{q}+\delta_{i}^{q(1-\beta)+\beta / 2}\right) .
$$

Thus for any $\xi_{i} \in \partial \Omega$ and any $q>1$,

$$
\left\|\frac{\partial Z_{i}}{\partial \nu}\right\|_{L^{q}(\partial \Omega)} \leq C \delta_{i}^{\beta / 2 q}
$$

Hence by elliptic regularity theory, we obtain that for any $1<q<2$ and any $0<\theta<1 / q$,

$$
\left\|Z_{i}\right\|_{W^{1+\theta, q}(\Omega)} \leq C\left(\left\|-\Delta_{a} Z_{i}+Z_{i}\right\|_{L^{q}(\Omega)}+\left\|\frac{\partial Z_{i}}{\partial \nu}\right\|_{L^{q}(\partial \Omega)}\right) \leq C \delta_{i}^{\beta(1 / q-1 / 2)} .
$$

Then by Morrey's embedding theorem,

$$
\left\|Z_{i}\right\|_{C^{\tau}(\bar{\Omega})} \leq C \delta_{i}^{\beta(1 / q-1 / 2)}
$$

where $0<\tau<1 / 2+1 / q$, which implies that expansion (2.19) holds with $\alpha=2 \beta(1 / q-1 / 2)$.

Proof of lemma 2.5. Notice that if we make the change of variables $s=1 / p$, then system $(2.22)$ can be rewritten in the following vector form

$$
S_{i}(s, \xi, \mu):=\log \mu_{i}+\frac{\log 8+\frac{1}{4} C_{1}+\frac{1}{4} C_{2} s}{4\left(1-\frac{1}{4} C_{1} s-\frac{1}{4} C_{2} s^{2}\right)}-\frac{1}{4} c_{i} H\left(\xi_{i}, \xi_{i}\right)-\frac{1}{4} \sum_{k=1, k \neq i}^{m}\left(\frac{\mu_{i}}{\mu_{k}}\right)^{2 s /(1-s)} c_{k} G\left(\xi_{i}, \xi_{k}\right)=0 .
$$


Obviously, from the explicit expression (2.15) of the constant $C_{1}$, we have that for $s=0$,

$$
\mu_{i}(0, \xi)=e^{-\frac{3}{4}+\frac{1}{4} c_{i} H\left(\xi_{i}, \xi_{i}\right)+\frac{1}{4} \sum_{k=1, k \neq i}^{m} c_{k} G\left(\xi_{i}, \xi_{k}\right)} .
$$

Using the Taylor expansion of exponential functions, we can conclude that for any $s>0$ small enough,

$$
\left(\frac{C^{2}}{s^{\kappa}}\right)^{2 s /(1-s)}=e^{\frac{2 s}{1-s}\left(2 \log C+\kappa \log \frac{1}{s}\right)}=1+O\left(s \log \frac{1}{s}\right),
$$

and then, by (2.23),

$$
\left(\frac{\mu_{i}}{\mu_{k}}\right)^{2 s /(1-s)}=1+O\left(s \log \frac{1}{s}\right), \quad \forall i \neq k .
$$

Moreover, by (1.7), (2.2) and the fact that $a\left(\xi_{i}\right) G\left(\xi_{i}, \xi_{k}\right)=a\left(\xi_{k}\right) G\left(\xi_{k}, \xi_{i}\right)$ for all $i, k=1, \ldots, m$ with $i \neq k$, we can easily prove that for any points $\xi=\left(\xi_{1}, \ldots, \xi_{m}\right) \in \mathcal{O}_{1 / s}$,

$$
G\left(\xi_{i}, \xi_{k}\right)=O\left(\log \frac{1}{s}\right), \quad \forall i \neq k .
$$

Consequently, a simple computation shows that

$$
\frac{\partial S_{i}(s, \xi, \mu)}{\partial \mu_{i}}=\frac{1}{\mu_{i}}-\frac{s}{2 \mu_{i}(1-s)} \sum_{k=1, k \neq i}^{m}\left(\frac{\mu_{i}}{\mu_{k}}\right)^{2 s /(1-s)} c_{k} G\left(\xi_{i}, \xi_{k}\right)=\frac{1}{\mu_{i}}\left[1+O\left(s \log \frac{1}{s}\right)\right],
$$

and for any $k \neq i$,

$$
\frac{\partial S_{i}(s, \xi, \mu)}{\partial \mu_{k}}=\frac{s}{2 \mu_{k}(1-s)}\left(\frac{\mu_{i}}{\mu_{k}}\right)^{2 s /(1-s)} c_{k} G\left(\xi_{i}, \xi_{k}\right)=\frac{1}{\mu_{k}} O\left(s \log \frac{1}{s}\right) .
$$

Then

$$
\operatorname{det}\left(\nabla_{\mu} S(s, \xi, \mu)\right)=\frac{1}{\mu_{1} \ldots \mu_{m}}\left[1+O\left(s \log \frac{1}{s}\right)\right] \neq 0 .
$$

Hence $\nabla_{\mu} S(s, \xi, \mu)$ is invertible in the range of points and variables that we are considering. From the Implicit Function Theorem we find that $S(s, \xi, \mu)=0$ is solvable in some neighborhood of $(0, \xi, \mu(0, \xi))$, and thus for any points $\xi=\left(\xi_{1}, \ldots, \xi_{m}\right) \in \mathcal{O}_{p}$ and any $p>1$ large enough, system $(2.22)$ has a unique solution $\mu=\left(\mu_{1}, \ldots, \mu_{m}\right)$ satisfying (2.23). This, together with (8.1)-(8.3), implies

$$
\mu_{i}=e^{-\frac{3}{4}+\frac{1}{4} c_{i} H\left(\xi_{i}, \xi_{i}\right)+\frac{1}{4} \sum_{k=1, k \neq i}^{m} c_{k} G\left(\xi_{i}, \xi_{k}\right)}\left[1+O\left(\frac{\log ^{2} p}{p}\right)\right], \quad \forall i=1, \ldots, m .
$$

Moreover, by (2.2), (2.4), (2.7), (2.22), (8.2) and (8.3) we can conclude that estimate (2.24) holds.

\section{REFERENCES}

[1] Adimurthi, G. Mancini, The Neumann problem for elliptic equations with critical nonlinearity, "A tribute in honour of G. Prodi", Scuola Norm. Sup. Pisa (1991) 9-25.

[2] Adimurthi, G. Mancini, Geometry and topology of the boundary in the critical Neumann problem, J. Reine Angew. Math. 456 (1994) 1-18.

[3] Adimurthi, G. Mancini, S.L. Yadava, The role of the mean curvature in semilinear Neumann problem involving critical exponent, Comm. Partial Differential Equations 20 (1995) 591-631.

[4] Adimurthi, F. Pacella, S.L. Yadava, Interaction between the geometry of the boundary and positive solutions of a semilinear Neumann problem with critical nonlinearity, J. Funct. Anal. 113 (1993) 318-350.

[5] O. Agudelo, A. Pistoia, Boundary concentration phenomena for the higher-dimensional Keller-Segel system, Calc. Var. Partial Differential Equations (2016) 55:132.

[6] S. Baraket and F. Parcard, Construction of singular limits for a semilinear elliptic equation in dimension 2, Calc. Var. Partial Differential Equations 6 (1998) 1-38.

[7] K.-C. Chang, Methods in Nonlinear Analysis, Springer-Verlag, Berlin, Heidelberg, New York, 2005. 
[8] D. Chae, O. Imanuvilov, The existence of non-topological multivortex solutions in the relativistic self-dual Chern-Simons theory, Comm. Math. Phys. 215 (2000) 119-142.

[9] C.C. Chen, C.S. Lin, Sharp estimates for solutions of multi-bubbles in compact Riemann surfaces, Comm. Pure Appl. Math. 55 (2002) 728-771.

[10] D. Cao, E.S. Noussair, S. Yan, Existence and nonexistence of interior-peaked solution for a nonlinear Neumann problem, Pacific J. Math. 200 (2001) 19-42.

[11] D. Cao, E.S. Noussair, The effect of geometry of the domain boundary in an elliptic Neumann problem, Adv. Differential Equations 6 (2001) 931-958.

[12] G. Cerami, J. Wei, Multiplicity of multiple interior peak solutions for some singularly perturbed Neumann problems, Internat. Math. Res. Notices 12 (1998) 601-626.

[13] M. del Pino, P.L. Felmer, J. Wei, On the role of mean curvature in some singularly perturbed Neumann problems, SIAM J. Math. Anal. 31 (1999) 63-79.

[14] M. del Pino, P.L. Felmer, J. Wei, On the role of distance function in some singular perturbation problems, Comm. Partial Differential Equations 25 (2000) 155-177.

[15] M. del Pino, M. Kowalczyk, M. Musso, Singular limits in Liouville-type equations, Calc. Var. Partial Differential Equations 24 (2005) 47-81.

[16] M. del Pino, F. Mahmoudi, M. Musso, Bubbling on boundary submanifolds for the Lin-Ni-Takagi problem at higher critical exponents, J. Eur. Math. Soc. 16 (2014) 1687-1748.

[17] M. del Pino, M. Musso, A. Pistoia, Super-critical boundary bubbling in a semilinear Neumann problem, Ann. Inst. H. Poincaré Analyse Non Linéaire 22 (2005) 45-82.

[18] M. del Pino, J. Wei, Collapsing steady states of the Keller-Segel system, Nonlinearity 19 (2006) 661-684.

[19] S. Deng, F. Mahmoudi, M. Musso, Bubbling on boundary submanifolds for a semiliear Neumann problem near higher critical exponents, Discrete Contin. Dynam. Systems A 36 (2016) 3035-3076.

[20] E.N. Dancer, S. Yan, Multipeak solutions for a singularly perturbed Neumann problem, Pacific J. Math. 189 (1999) 241-262.

[21] P. Esposito, Interior estimates for some semilinear elliptic problem with critical nonlinearity, Ann. Inst. H. Poincaré Analyse Non Linéaire 24 (2007) 629-644.

[22] P. Esposito, M. Musso, A. Pistoia, Concentrating solutions for a planar elliptic problem involving nonlinearities with large exponent, J. Differential Equations 227 (2006) 29-68.

[23] A. Gierer, H. Meinhardt, A theory of biological pattern formation, Kybernetik(Berlin) 12 (1972) 30-39.

[24] M. Grossi, A class of solutions for the Neumann problem $-\Delta u+\lambda u=u^{\frac{N+2}{N-2}}$, Duke Math. J. 79 (1995) 309-335.

[25] M. Grossi, A. Pistoia, J. Wei, Existence of multipeak solutions for a semilinear Neumann problem via nonsmooth critical point theory, Calc. Var. Partial Differential Equations 11 (2000) 143-175.

[26] N. Ghoussoub, C. Gui, M. Zhu, On a singularly perturbed Neumann problem with critical exponent, Comm. Partial Differential Equation 26 (2001) 1929-1946.

[27] C. Gui, N. Ghoussoub, Multi-peak solutions for a semilinear Neumann problem involving the critical Sobolev exponent, Math. Z. 229 (1998) 443-474.

[28] C. Gui, C.S. Lin, Estimates for boundary-bubbling solutions to an elliptic Neumann problem, J. Reine Angew. Math. 546 (2002) 201-235.

[29] C. Gui, J. Wei, Multiple interior spike solutions for some singular perturbed Neumann problems, J. Differential Equations, 158 (1999) 1-27.

[30] C. Gui, J. Wei, On multiple mixed interior and boundary peak solutions for some singularly perturbed Neumann problems, Canad. J. Math. 52 (2000) 522-538.

[31] C. Gui, J. Wei, M. Winter, Multiple boundary peak solutions for some singularly perturbed Neumann problems, Ann. Inst. H. Poincaré Anal. Non Linéaire, 17 (2000) 249-289.

[32] M. Kowalczyk, Multiple spike layers in the shadow Gierer-Meinhardt system: existence of equilibria and the quasi-invariant manifold, Duke Math. J. 98 (1999) 59-111.

[33] E.F. Keller, L.A. Segel, Initiation of slime mold aggregation viewed as an instability, J. Theoretical Biology 26 (1970) 399-415.

[34] C.S. Lin, W.M. Ni, I. Takagi, Large amplitude stationary solutions to a chemotaxis system, J. Differential Equations 72 (1988) 1-27.

[35] C.S. Lin, L. Wang, J. Wei, Bubble accumulations in an elliptic Neumann problem with critical Sobolev exponent, Calc. Var. Partial Differential Equations 30 (2007) 153-182. 
[36] Y.-Y. Li, On a singularly perturbed equation with Neumann boundary condition, Comm. Partial Differential Equations 23 (1998) 487-545.

[37] N. G. Lloyd, Degree theory, Cambridge Universary Press, 1977.

[38] F. Mahmoudi, A. Malchiodi, Concentration on minimal submanifolds for a singularly perturbed Neumann problem, Adv. Math. 209 (2007) 460-525.

[39] A. Malchiodi, Concentration at curves for a singularly perturbed Neumann problem in three-dimensional domains, Geom. Funct. Anal. 15 (2005) 1162-1222.

[40] A. Malchiodi, M. Montenegro, Boundary concentration phenomena for a singularly perturbed elliptic problem, Comm. Pure Appl. Math. 55 (2002) 1507-1568.

[41] A. Malchiodi, M. Montenegro, Multidimensional boundary-layers for a singularly perturbed Neumann problem, Duke Math. J. 124 (2004) 105-143.

[42] B. Manna, A. Pistoia, Boundary-layers for a Neumann problem at higher critical exponents, Boll. Unione Mat. Ital. 10 (2017) 355-368.

[43] M. Musso, J. Wei, Stationary solutions to a Keller-Segel chemotaxis system, Asymptotic Anal. 49 (2006) $217-247$.

[44] W.M. Ni, Diffusion, cross-diffusion, and their spike-layer steady states, Notices Amer. Math. Soc. 45 (1998) 9-18.

[45] W.N. Ni, X.B. Pan, I. Takagi, Singular behavior of least-energy solutions of a semi-linear Neumann problem involving critical Sobolev exponents, Duke Math. J. 67 (1992) 1-20.

[46] W.M. Ni, I. Takagi, On the shape of least-energy solutions to a semilinear Neumann problem, Comm. Pure Appl. Math. 44 (1991) 819-851.

[47] W.M. Ni, I. Takagi, Locating the peaks of least-energy solutions to a semilinear Neumann problem, Duke Math. J. 70 (1993) 247-281.

[48] M. H. Protter, H. F. Weinberger, Maximum Principles in Differential Equation, Corrected Reprint of the 1967 Original, Springer-Verlag, New York, 1984.

[49] O. Rey, Boundary effect for an elliptic Neumann problem with critical nonlinearity, Comm. Partial Differential Equations 22 (1997) 1055-1139.

[50] O. Rey, An elliptic Neumann problem with critical nonlinearity in three dimensional domains, Comm. Contemp. Math. 1 (1999) 405-449.

[51] O. Rey, The question of interior blow-up points for an elliptic Neumann problem: the critical case, J. Math. Pures Appl. 81 (2002) 655-696.

[52] O. Rey, J. Wei, Blow-up solutions for an elliptic Neumann problem with sub-or-supcritical nonlinearity, II: $N=4$, Ann. Inst. H. Poincaré Analyse Non Linéaire 22 (2005) 459-484.

[53] O. Rey, J. Wei, Blow-up solutions for an elliptic Neumann problem with sub-or-supcritical nonlinearity, I: $N=3$, J. Funct. Anal. 212 (2004) 472-499.

[54] L. Wang, Arbitrarily many solutions for an elliptic Neumann problem with sub- or supercritical nonlinearity, Commun. Pure Appl. Anal. 9 (2010) 761-778.

[55] X.J. Wang, Neumann problem of semilinear elliptic equations involving critical Sobolev exponents, J. Differential Equations 93 (1991) 283-310.

[56] J. Wei, On the boundary spike layer solutions of singularly perturbed semilinear Neumann problem, J. Differential Equations 134 (1997) 104-133.

[57] J. Wei, On the interior spike layer solutions to a singularly perturbed Neumann problem, Tohoku Math. J. 50 (1998) $159-178$.

[58] J. Wei, M. Winter, Multiple boundary spike solutions for a wide class of singular perturbation problems, J. Lond. Math. Soc. 59 (1999) 585-606.

[59] J. Wei, S. Yan, Arbitrary many boundary peak solutions for an elliptic Neumann problem with critical growth, J. Math. Pures Appl. 88 (2007) 350-378.

College of Sciences, Nanjing Agricultural University, Nanjing 210095, China

Email address: yibin10201029@njau.edu.cn 